ELECTRONIC DONATION OR STERIC CONTRACTION: A SPECTROSCOPIC AND STRUCTURAL ANALYSIS OF MEDIUM-SIZED CONSTRAINED RINGS FOR POTENTIAL LONG-RANGE HYPERCONJUGATION

Robert Lee ${ }^{\S}$, Bryan Bashrum ${ }^{\S}$, Ethan C. Cagle ${ }^{\ddagger}$, Jillian Walters ${ }^{\S}$, Jake Massey ${ }^{\ddagger}$, Monica Zanghi ${ }^{\ddagger}$, Carolyn Birchfield ${ }^{\ddagger}$, David French ${ }^{\ddagger}$, Jessica Joy $^{\ddagger}$, Gabriel dos Passos Gomes ${ }^{¥, \mathrm{a}}$,Paul A. Wiget ${ }^{\S *}$

${ }^{\S}$ Department of Chemistry and Biochemistry, Samford University, 800 Lakeshore Blvd., Birmingham, Al 35229

${ }^{\neq}$Department of Biological and Environmental Sciences, Samford University, Birmingham, Al

${ }^{¥}$ Department of Chemistry \& Biochemistry, Florida State University, Tallahassee, Fl

a Present address: Department of Chemistry, University of Toronto, Toronto, ON, Canada,

${ }^{\notin}$ Department of Chemistry, The University of Alabama at Birmingham, Birmingham, Al

*pwiget@samford.edu

Table of Contents

Table of Contents.

Data 1'

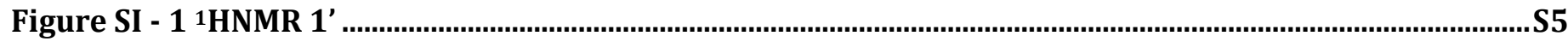

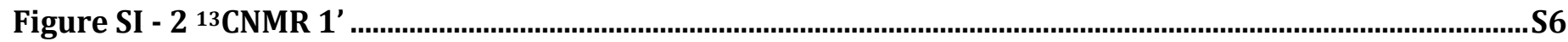

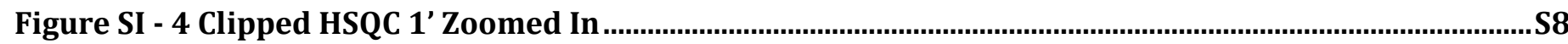

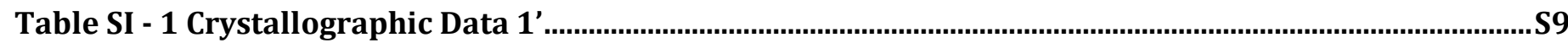

Figure SI - 5 X-Ray crystal structure. Atomic displacement ellipsoids generated at 50\% probability........S9

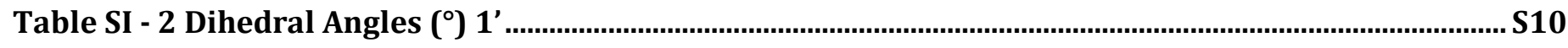

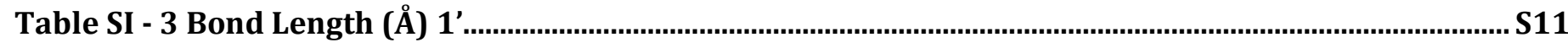

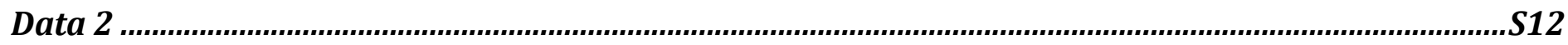

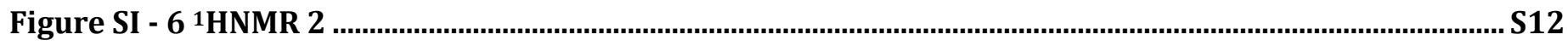

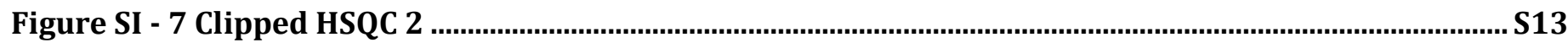

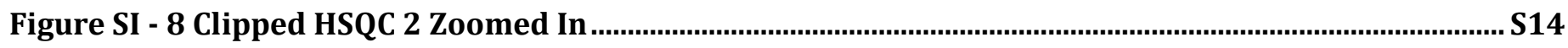

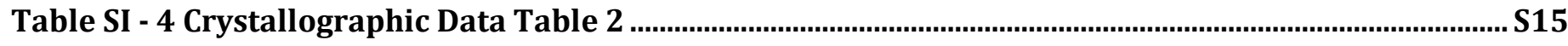

Figure SI - 9 X-Ray crystal structure. Atomic displacement ellipsoids generated at 50\% probability .....S15

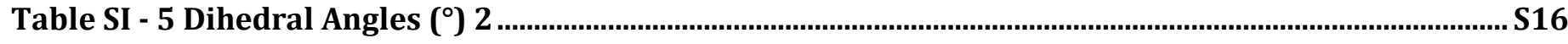

Table SI - 6 Bond Length $(\AA ̊)$

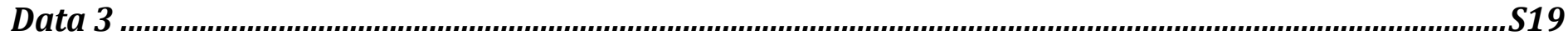

Figure SI - 10 1HNMR 3

Figure SI - 11 13CNMR 3

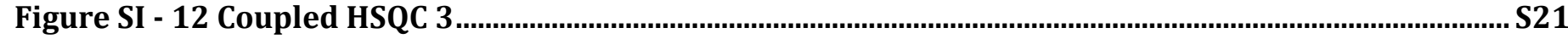

Figure SI - 13 Clipped HSQC 3

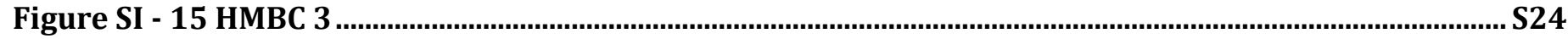

Figure SI - 16 NOESY 3

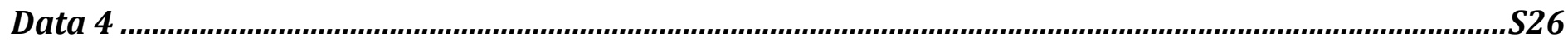

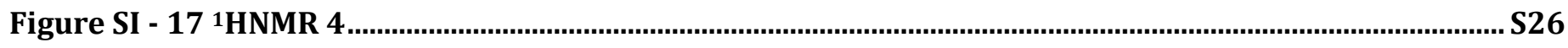




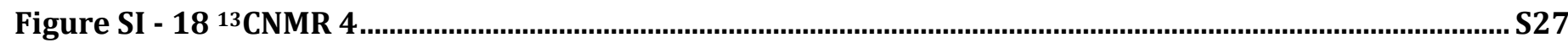

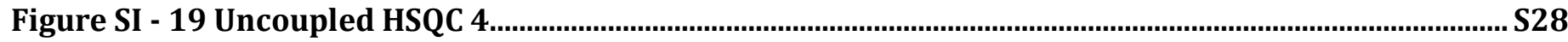

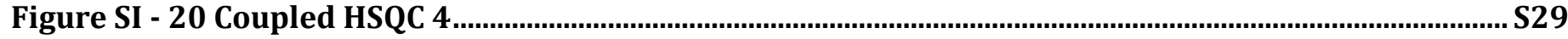

Figure SI - 21 Clipped HSQC 4

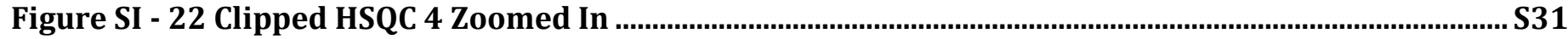

Figure SI - 23 HMBC 4

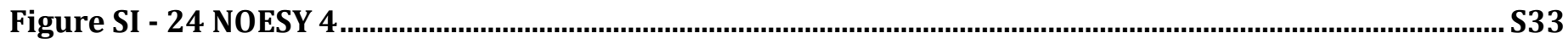

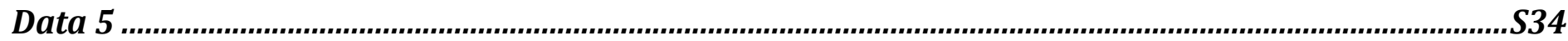

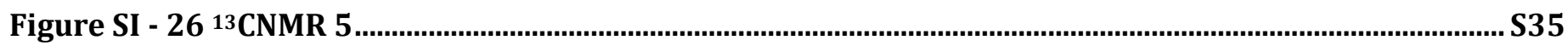

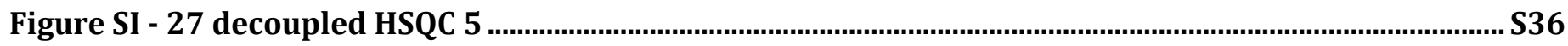

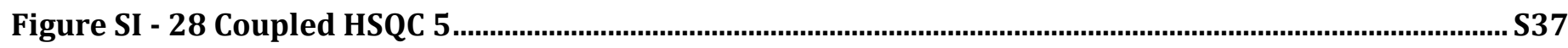

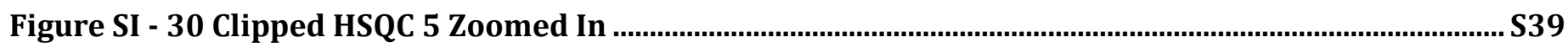

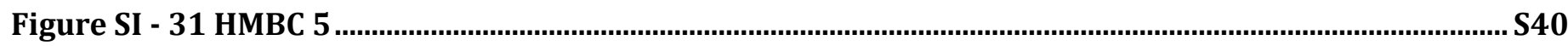

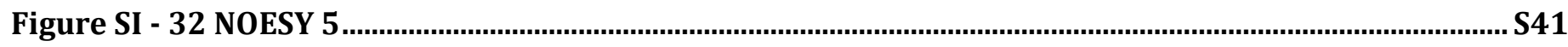

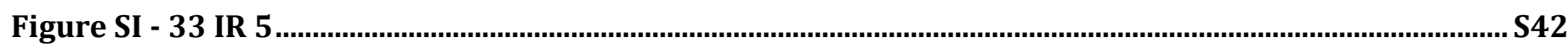

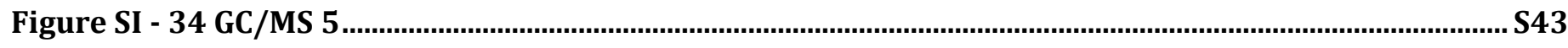

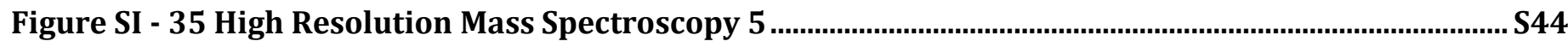

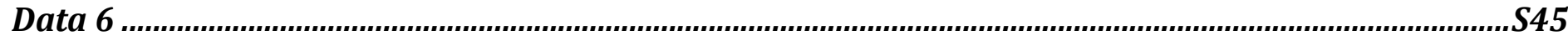

Figure SI - 36 1HNMR 6

Figure SI - $37{ }^{13}$ CNMR 6

Figure SI - 38 Coupled HSQC 6

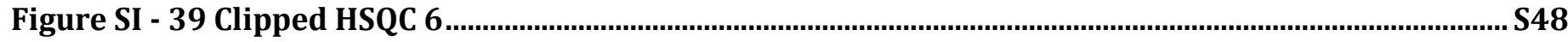

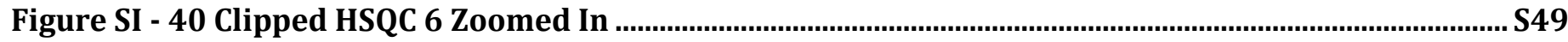

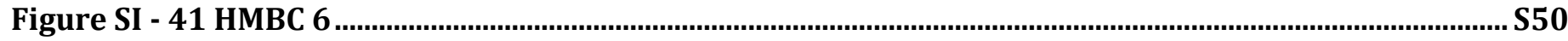

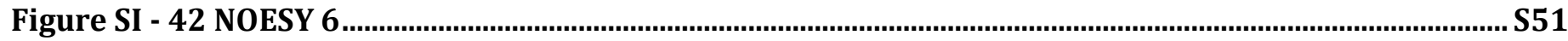

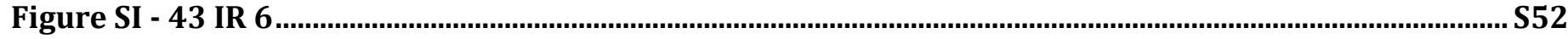

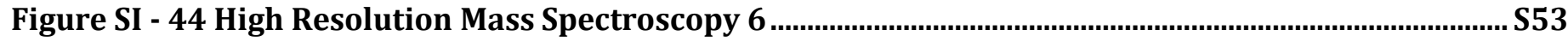

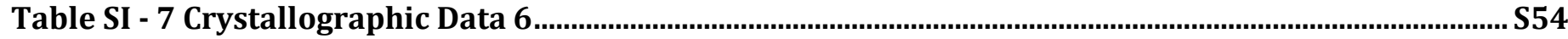

Figure SI - 45 S3 X-Ray crystal structure. Atomic displacement ellipsoids generated at 50\% probability

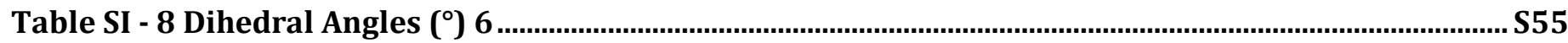

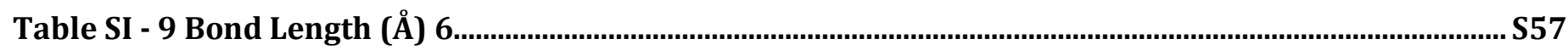

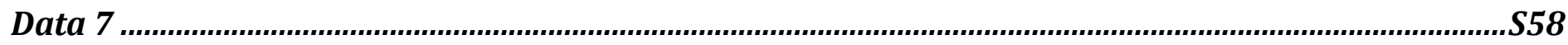

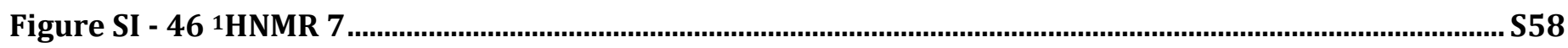

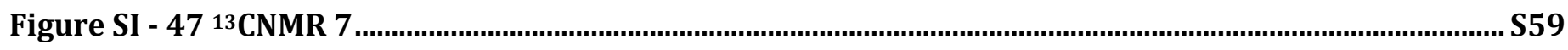

Figure SI - 48 Uncoupled HSQC 7

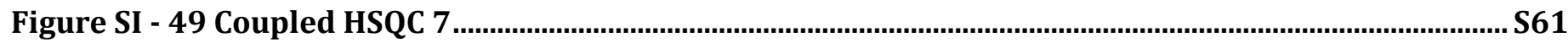

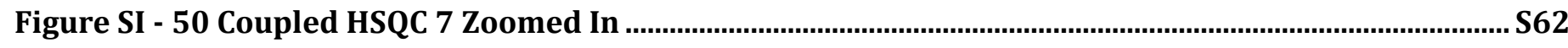




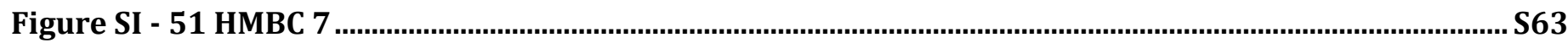

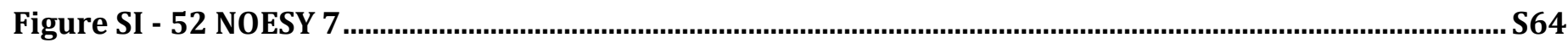

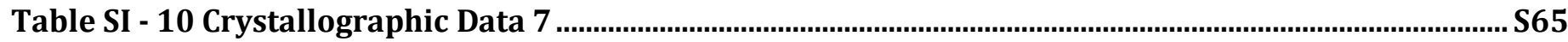

Figure SI - 53 X-Ray crystal structure 7. Atomic displacement ellipsoids generated at 50\% probabilityS65

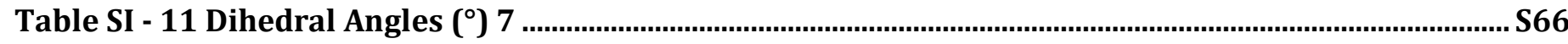

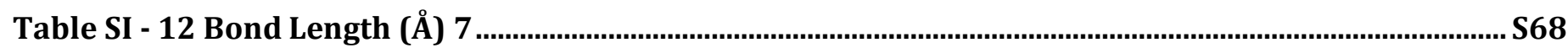

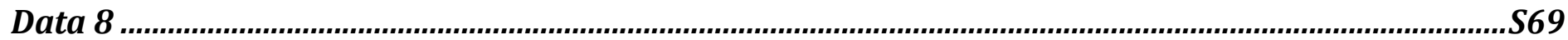

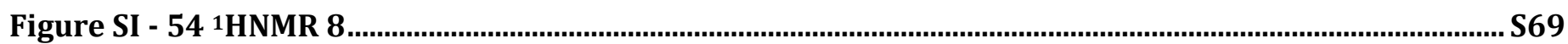

Figure SI - 55 13CNMR 8

Figure SI - 56 Coupled HSQC 8

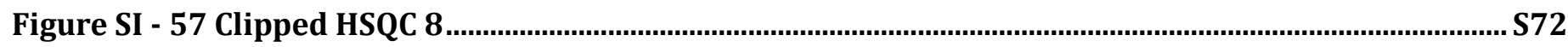

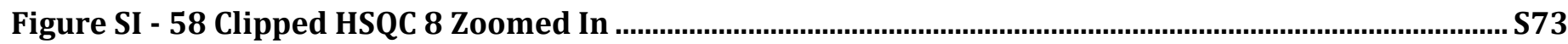

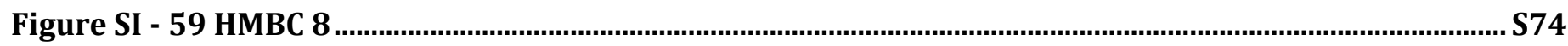

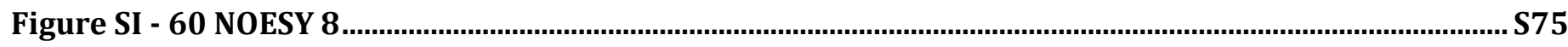

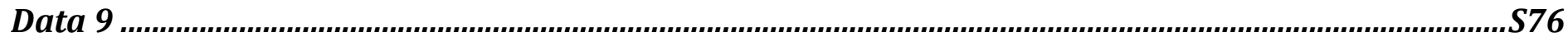

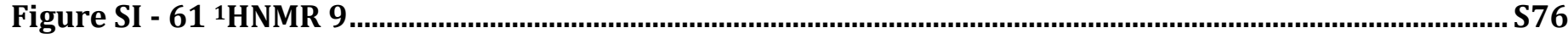

Figure SI - 63 Uncoupled HSQC 9

Figure SI - 64 Coupled HSQC 9

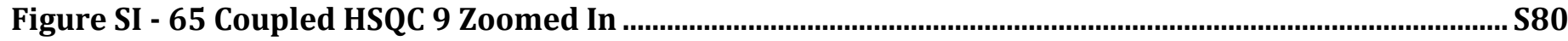

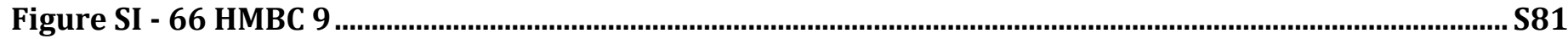

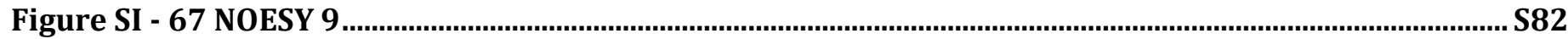

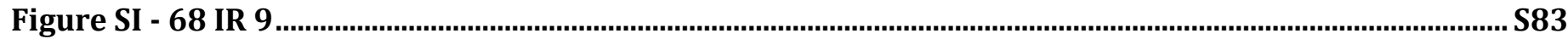

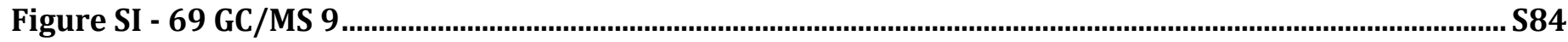

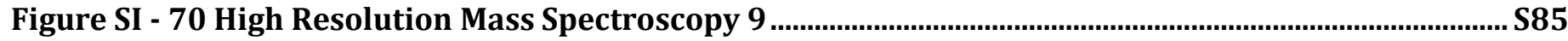

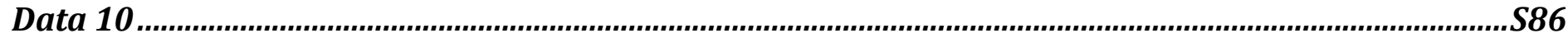

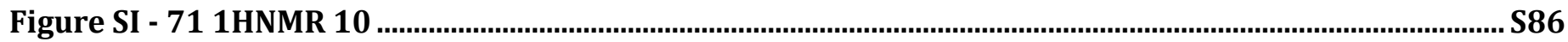

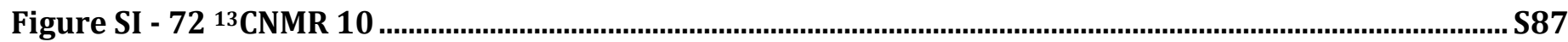

Figure SI - 73 HSQC 10

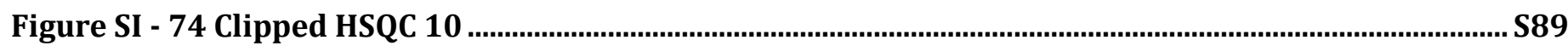

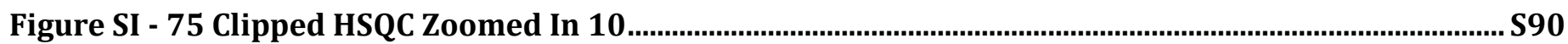

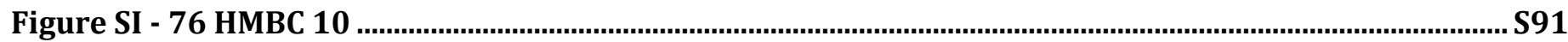

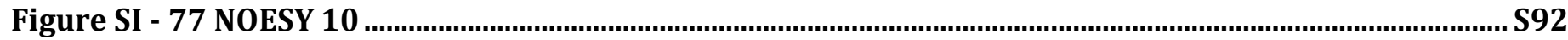

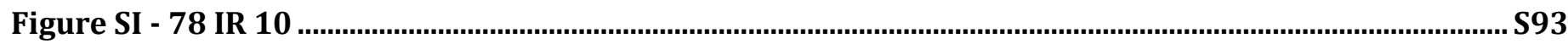

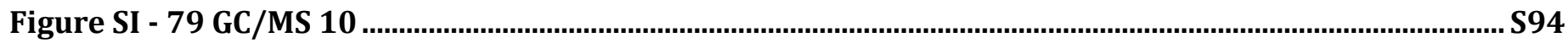

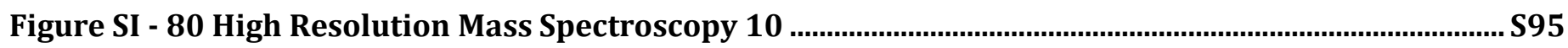

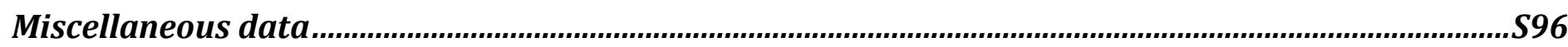

Figure SI - 81. PERFECT-CLIP ZOOM of 1' @ 298 in CD2Cl2 (top), Tol-d8 (bottom) ..................................S96

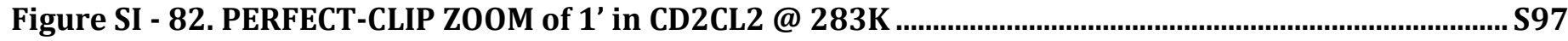


Figure SI - 83. PERFECT-CLIP of cyclohexanone (zoom) in CD2Cl2 @298K (top), 283K (bottom) ............. S98

Figure SI - 84. PERFECT-CLIP of cyclohexanone (zoom) in Tol-d8 @ 298K (top), 283K (bottom).............S99

Figure SI - 85. PERFECT-CLIP cyclohexanol zoom in Tol-d8 @298K.........................................................S100

Figure SI - 86. PERFECT-CLIP of cyclohexanol in Tol-d8 @ 277K ................................................................S101

Figure SI - 87. PERFECT-CLIP of cyclohexanol in CD2Cl2 @ 283K

Figure SI - 88. PERFECT-CLIP zoom of cyclohexnol in Tol-d8 @ 283K...............................................................S102

Figure SI - 89. PERFECT-CLIP of cyclohexanol in CD2Cl2 @ 298K...................................................................S103

Figure SI - 90. Plot axial versus equatorial coupling constants in all compounds. Red compounds are from ref $4 a$. 
రั0

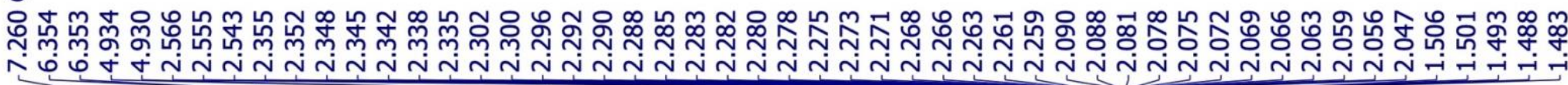

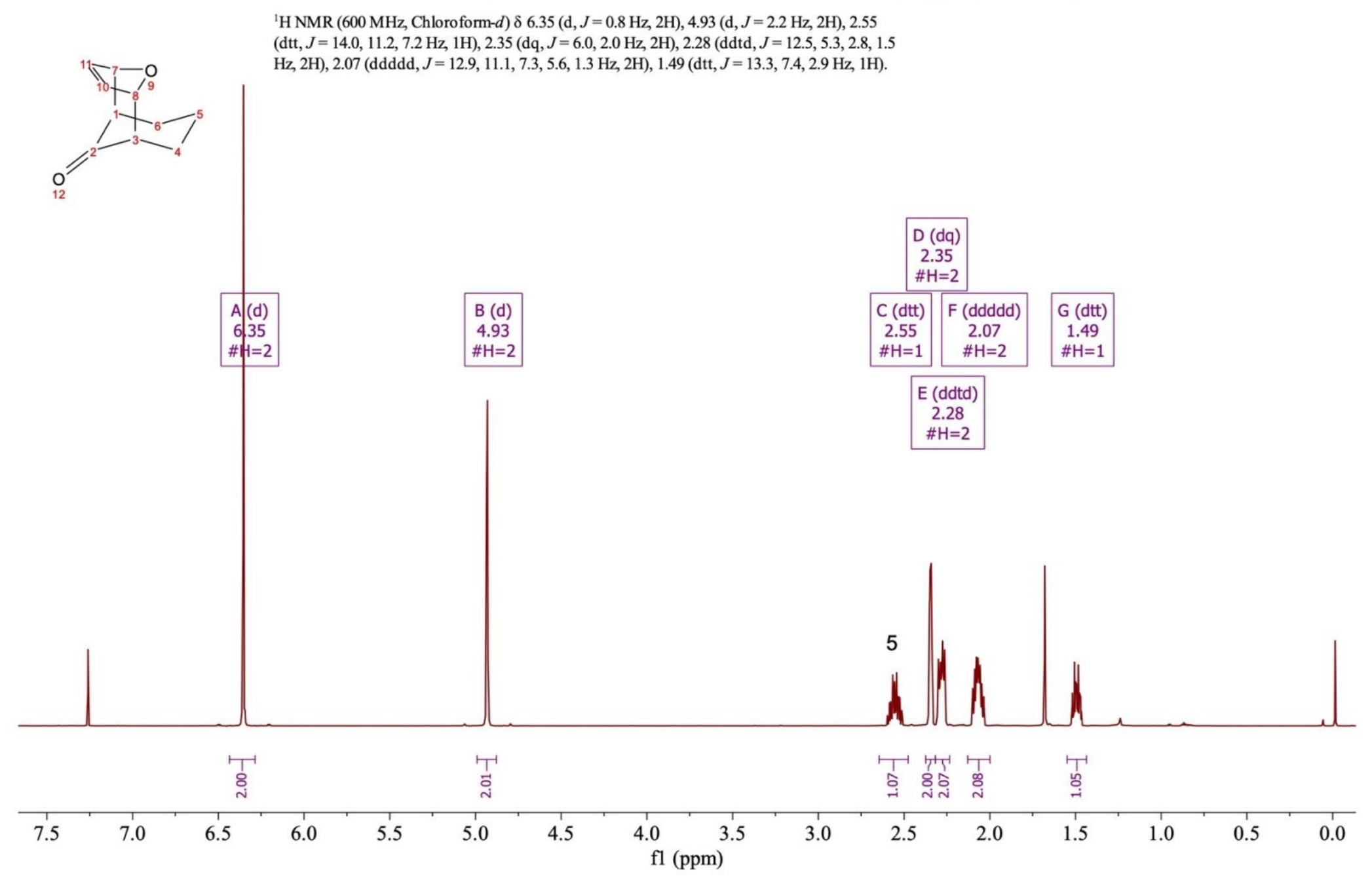



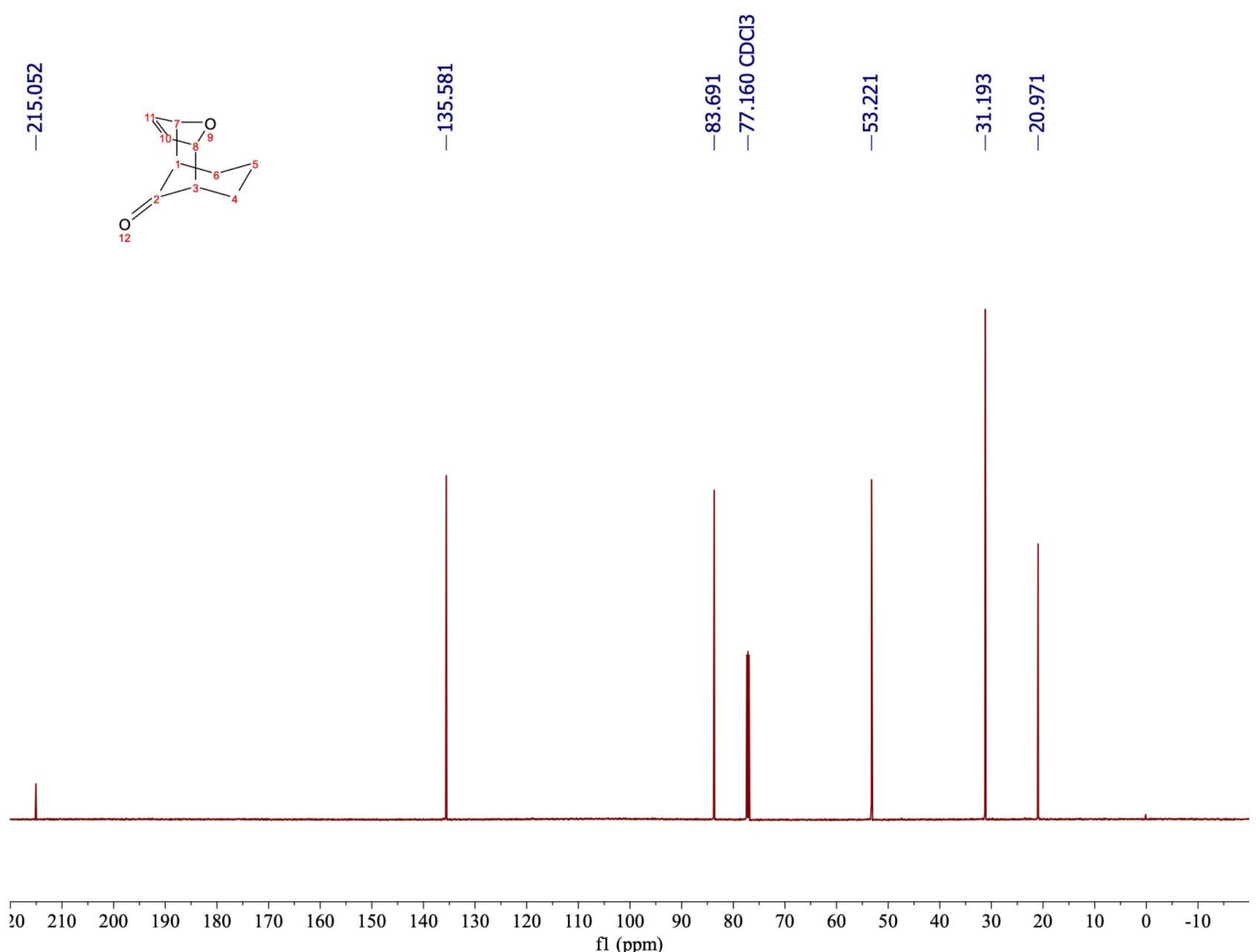


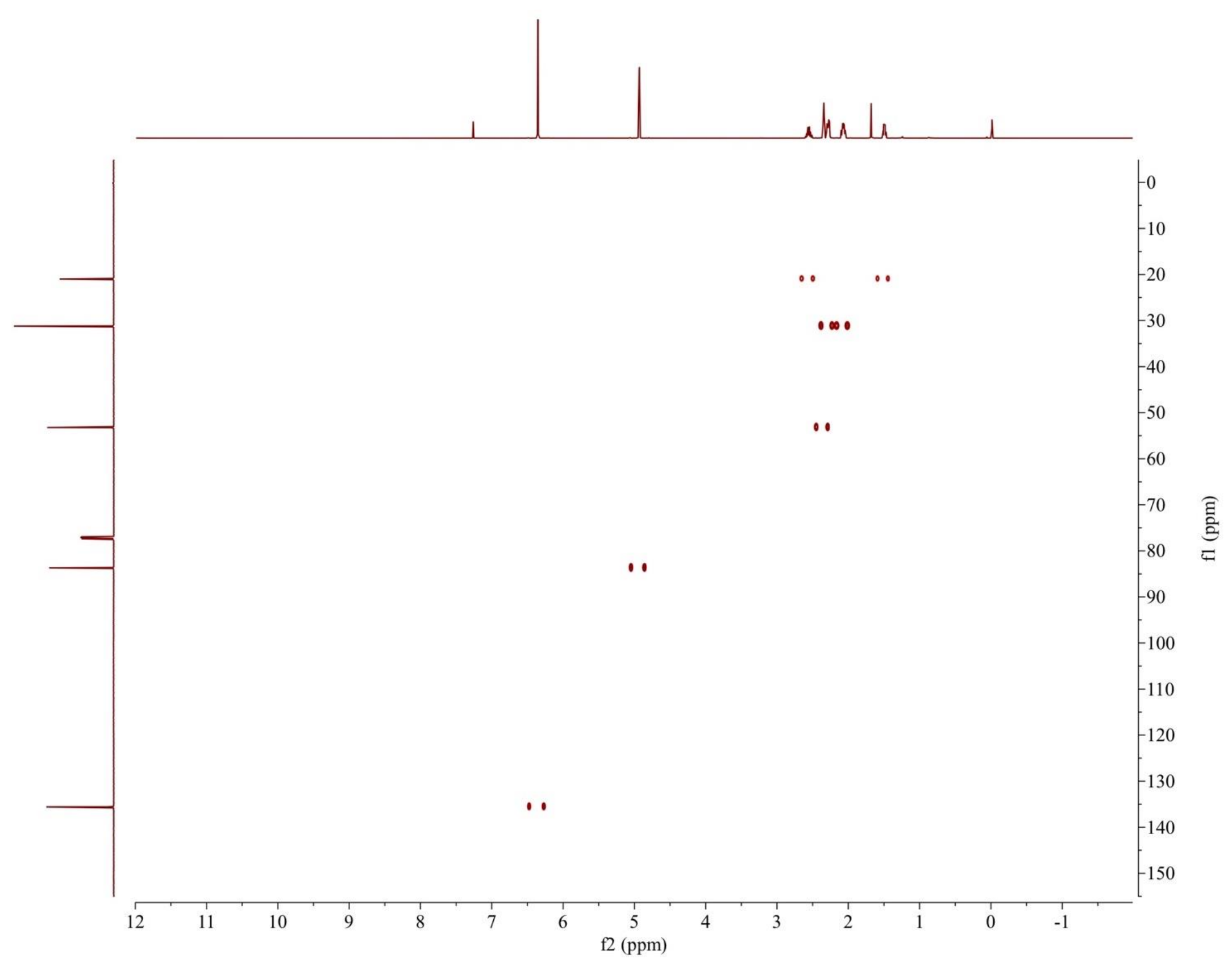




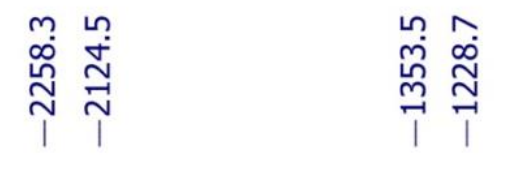

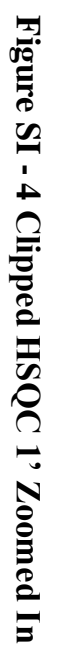

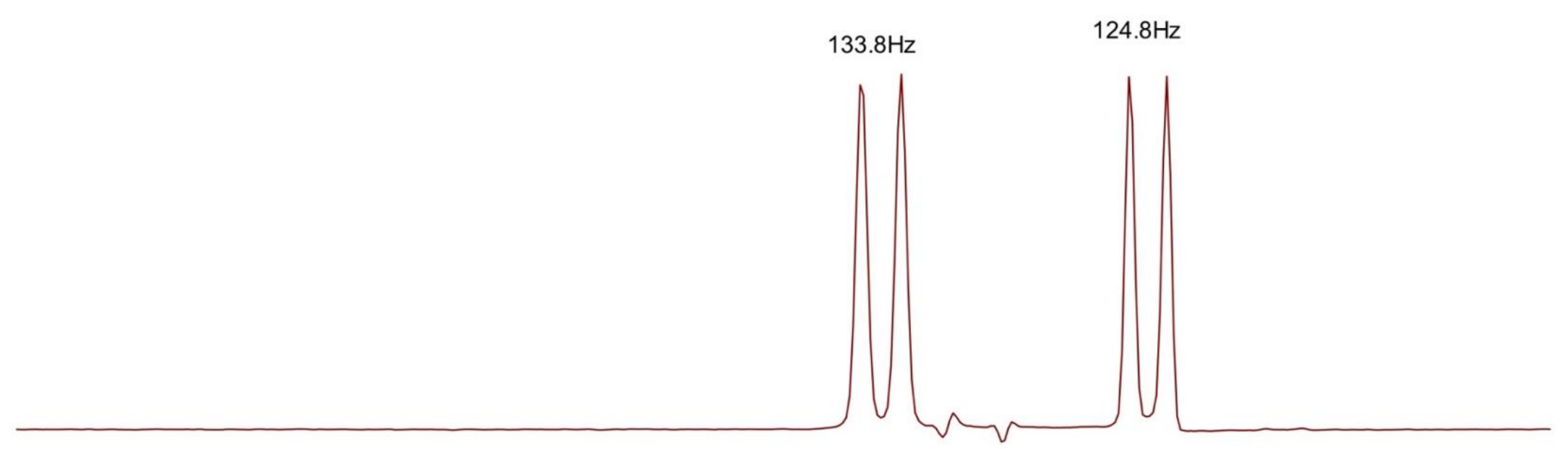

\begin{tabular}{lllllllllllllllllllllllllllllllllllllllllll}
\hline 0 & 5.8 & 5.6 & 5.4 & 5.2 & 5.0 & 4.8 & 4.6 & 4.4 & 4.2 & 4.0 & 3.8 & 3.6 & 3.4 & 3.2 & 3.0 & 2.8 & 2.6 & 2.4 & 2.2 & 2.0 & 1.8 & 1.6 & 1.4 & 1.2 & 1.0 & 0.8 & 0.6 & 0.4 & 0.2 & 0.0
\end{tabular} $\mathrm{fl}(\mathrm{ppm})$ 
Table SI - 1 Crystallographic Data 1'

\begin{tabular}{|c|c|}
\hline Empirical formula & $\mathrm{C}_{10} \mathrm{H}_{12} \mathrm{O}_{2}$ \\
\hline Formula weight & $164.20 \mathrm{~g} / \mathrm{mol}$ \\
\hline Temperature & $173 \mathrm{~K}$ \\
\hline Wavelength & $1.54178 \AA$ \\
\hline Crystal system & monoclinic \\
\hline Space group & $\mathrm{P} 2{ }_{1} / \mathrm{c}$ \\
\hline Unit cell dimensions & $\begin{array}{l}\mathrm{a}=9.04980(10) \AA, \alpha=90^{\circ} \\
\mathrm{b}=6.42590(10) \AA, \beta=100.9440(10)^{\circ} \\
\mathrm{c}=14.3599(10) \AA, \gamma=90^{\circ}\end{array}$ \\
\hline Volume & $819.89(2) \AA^{3}$ \\
\hline $\mathbf{Z}$ & 4 \\
\hline Density (calculated) & $1.330 \mathrm{~g} / \mathrm{cm}^{3}$ \\
\hline Absorption coefficient & $0.739 \mathrm{~mm}^{-1}$ \\
\hline $\mathbf{F}(\mathbf{0 0 0})$ & 352.0 \\
\hline$\theta_{\max }$ & $70.02^{\circ}$ \\
\hline $\begin{array}{l}\text { Reflections } \\
\text { collected }\end{array}$ & $\begin{array}{l}8932 \\
{\left[\mathrm{R}_{\text {int }}=2.26 \%\right]}\end{array}$ \\
\hline Independent reflections & 1553 \\
\hline Completeness to $\theta \max$ & $97.3 \%$ \\
\hline Min./max. transmission & 0.7420 \\
\hline Refinement method & Full matrix least squares on F2 \\
\hline Absorption correction & Numerical \\
\hline Goodness-of-fit & 1.064 \\
\hline Final $\mathbf{R}$ indices & $4.11 \%$ \\
\hline $\mathbf{R}$ indices (all) & $14.08 \%$ \\
\hline $\begin{array}{l}\text { Largest diff. peak } \\
\text { and hole }\end{array}$ & $0.28,-0.24 \mathrm{e}^{-} / \AA^{3}$ \\
\hline
\end{tabular}

Figure SI - 5 X-Ray crystal structure. Atomic displacement ellipsoids generated at $50 \%$ probability. Recrystallized from a minimum of hot hexanes.
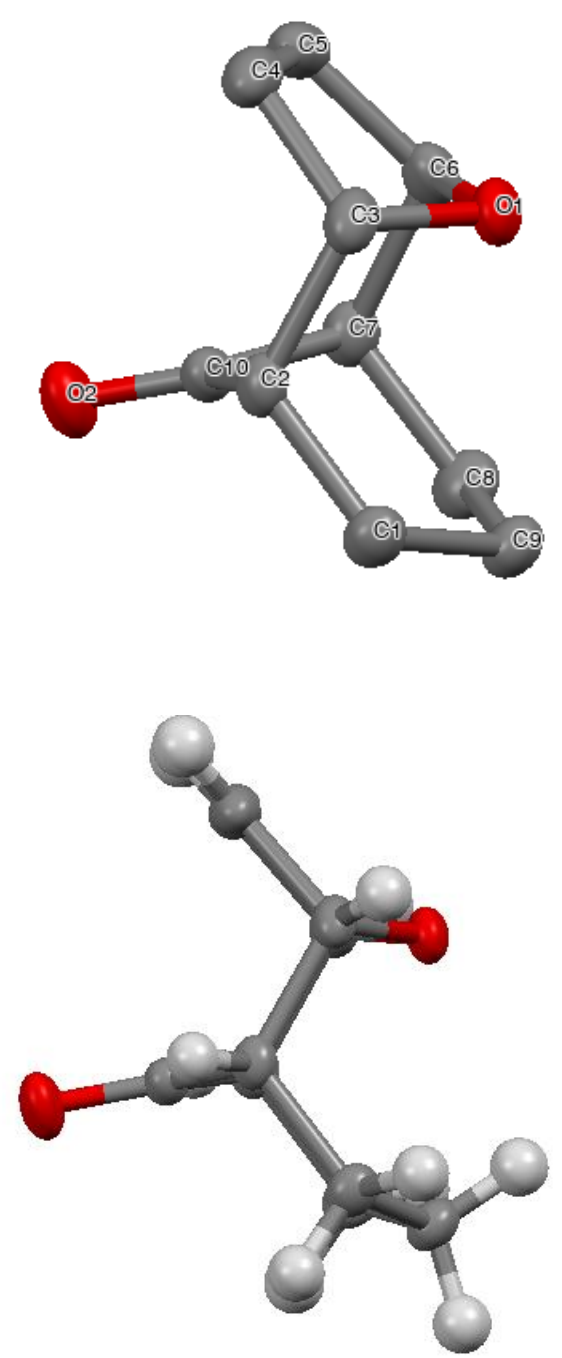
Table SI - 2 Dihedral Angles $\left({ }^{\circ}\right) 1$,

\begin{tabular}{|l|l|l|l|l|}
\hline Number & Atom1 & Atom2 & Atom3 & Angle $\left(^{\circ}\right)$ \\
\hline $\mathbf{1}$ & C6 & O1 & C3 & $102.30(8)$ \\
\hline $\mathbf{2}$ & C9 & C1 & C2 & $114.89(9)$ \\
\hline $\mathbf{4}$ & C1 & C2 & C3 & $113.12(9)$ \\
\hline $\mathbf{5}$ & C10 & C2 & C1 & $107.21(9)$ \\
\hline $\mathbf{6}$ & C10 & C2 & C3 & $106.85(9)$ \\
\hline $\mathbf{7}$ & O1 & C3 & C2 & $107.99(8)$ \\
\hline $\mathbf{8}$ & O1 4 & C3 & C4 & $101.83(9)$ \\
\hline $\mathbf{9}$ & C5 & C4 & C3 & $110.18(9)$ \\
\hline $\mathbf{1 0}$ & C4 & C5 & C6 & $107.33(11)$ \\
\hline $\mathbf{1 1}$ & O1 & C6 & C5 & $101.93(10)$ \\
\hline $\mathbf{1 2}$ & O1 & C6 & C7 & $108.05(9)$ \\
\hline $\mathbf{1 3}$ & C5 & C6 & C7 & $109.97(9)$ \\
\hline $\mathbf{1 4}$ & C8 & C7 & C6 & $113.82(9)$ \\
\hline $\mathbf{1 5}$ & C10 & C7 & C6 & $106.77(9)$ \\
\hline $\mathbf{1 6}$ & C10 & C7 & C8 & $106.73(9)$ \\
\hline $\mathbf{1 7}$ & C9 & C8 & C7 & $114.87(9)$ \\
\hline $\mathbf{1 8}$ & C8 & C9 & C1 & $115.02(9)$ \\
\hline $\mathbf{1 9}$ & O2 & C10 & C2 & $124.51(10)$ \\
\hline $\mathbf{2 0}$ & O2 & C10 & C7 & $124.54(10)$ \\
\hline $\mathbf{2 1}$ & C 510 & C7 & $110.93(9)$ \\
\hline
\end{tabular}


Table SI - 3 Bond Length (Å) 1'

\begin{tabular}{|l|l|l|l|}
\hline Number & Atom1 & Atom2 & Length \\
\hline $\mathbf{1}$ & O1 & C3 & $1.4371(14)$ \\
\hline $\mathbf{2}$ & O1 & C6 & $1.4324(14)$ \\
\hline $\mathbf{3}$ & O2 & C10 & $1.2126(15)$ \\
\hline $\mathbf{4}$ & C1 & C2 & $1.5449(15)$ \\
\hline $\mathbf{5}$ & C1 & C9 & $1.5432(15)$ \\
\hline $\mathbf{6}$ & C2 & C3 & $1.5569(15)$ \\
\hline $\mathbf{7}$ & C2 & C10 & $1.5107(14)$ \\
\hline $\mathbf{8}$ & C3 & C4 & $1.5165(16)$ \\
\hline $\mathbf{9}$ & C4 & C5 & $1.3248(19)$ \\
\hline $\mathbf{1 0}$ & C5 & C6 & $1.5169(17)$ \\
\hline $\mathbf{1 1}$ & C6 & C7 & $1.5565(15)$ \\
\hline $\mathbf{1 2}$ & C7 & C8 & $1.5451(15)$ \\
\hline $\mathbf{1 3}$ & C7 & C10 & $1.5103(15)$ \\
\hline $\mathbf{1 4}$ & C8 & C9 & $1.5414(16)$ \\
\hline
\end{tabular}




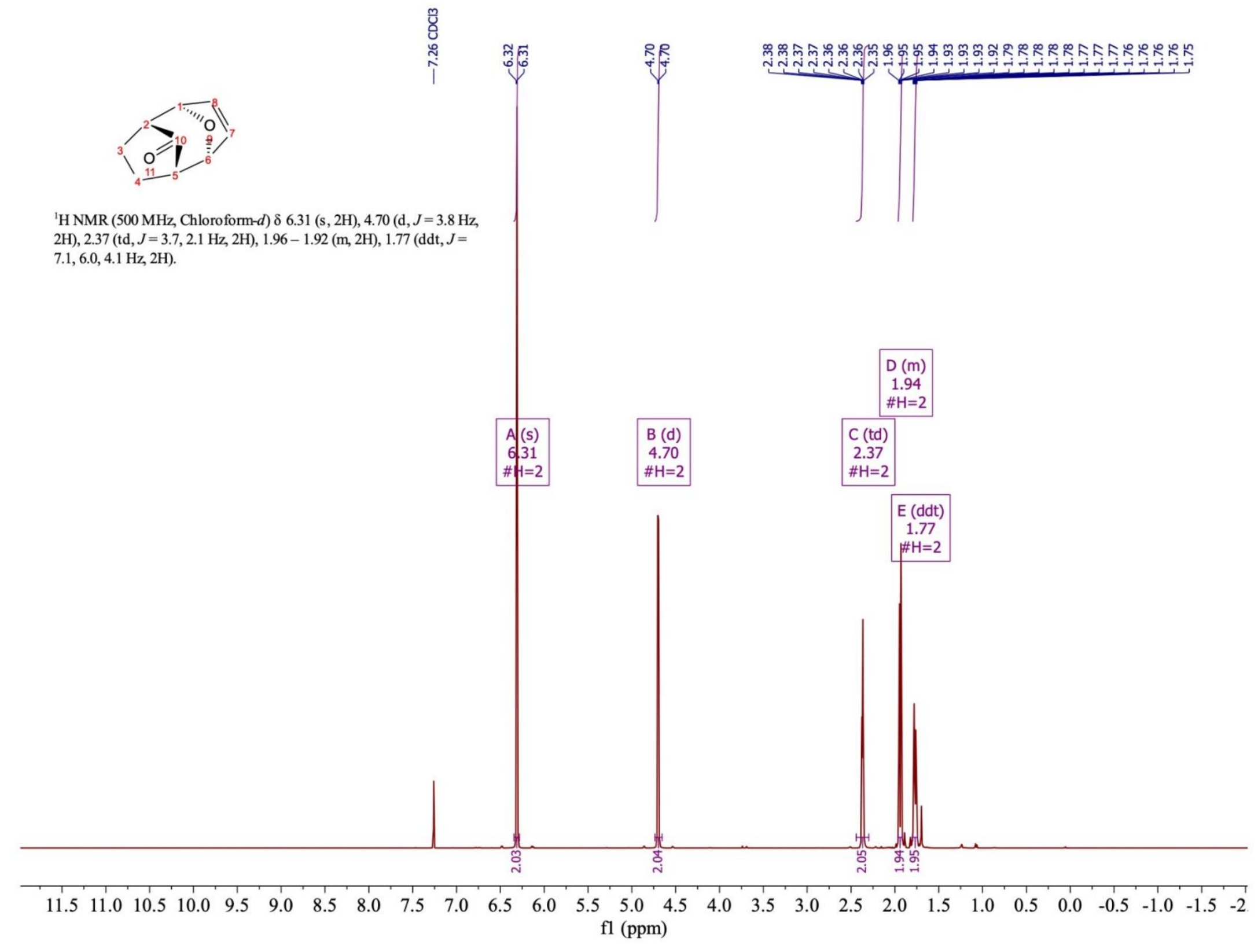




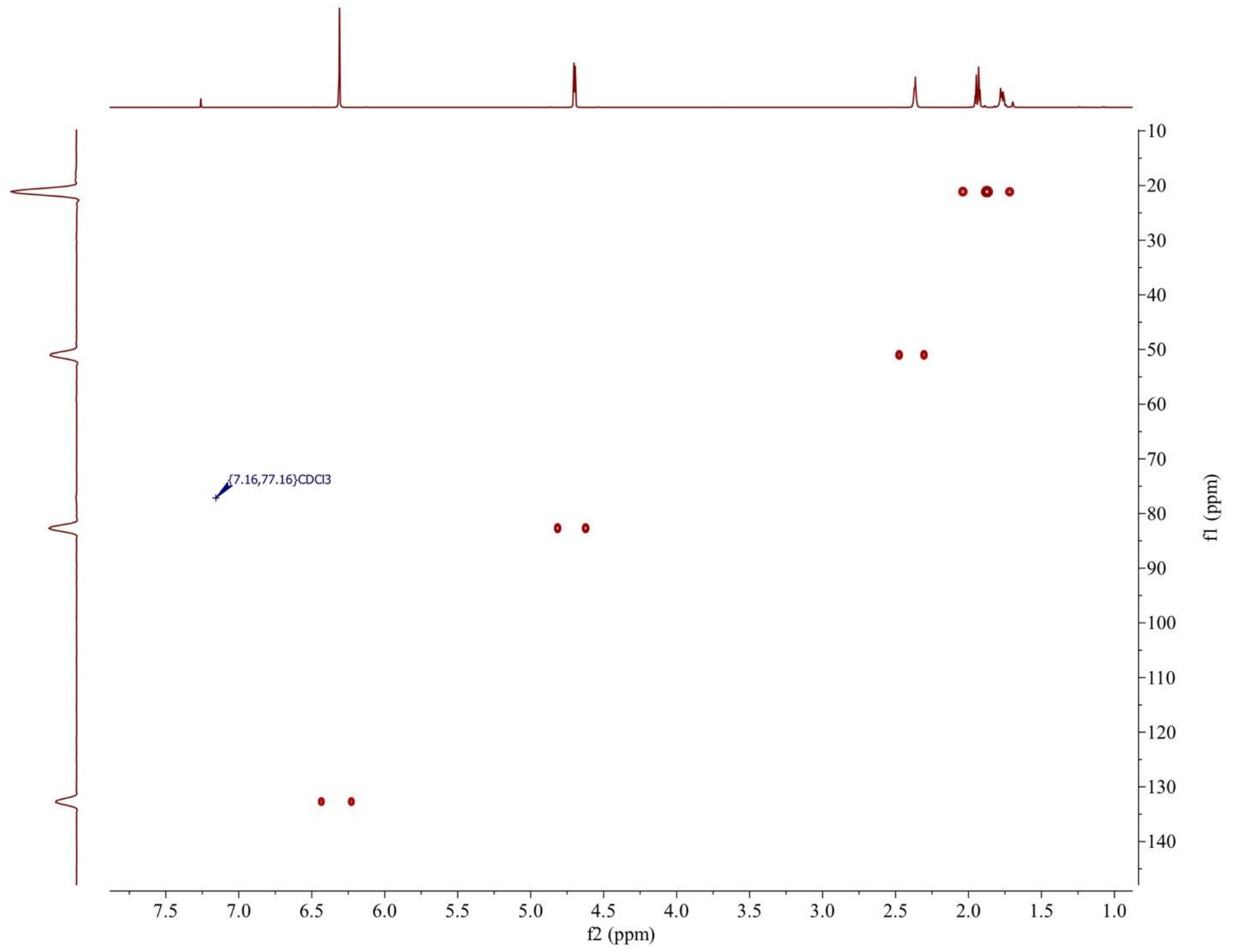

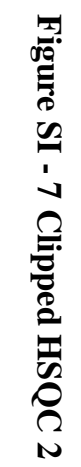




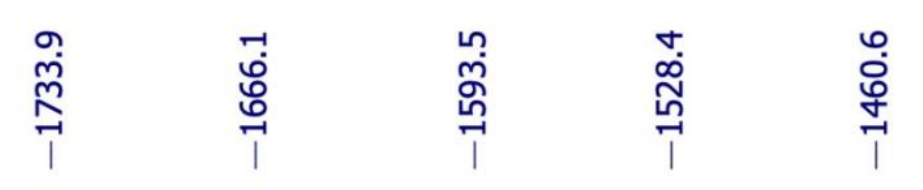

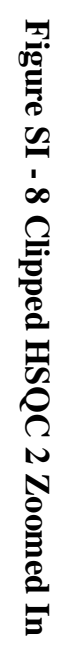
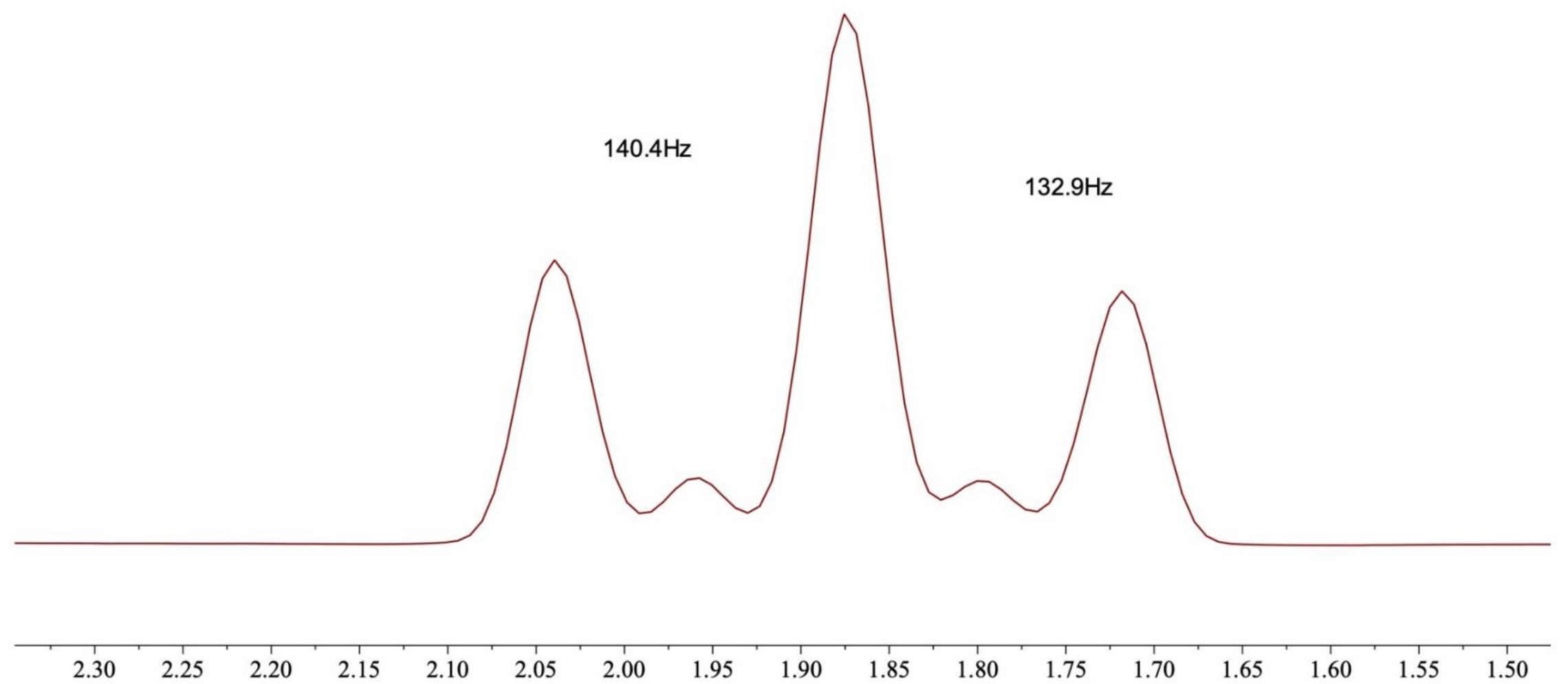
Table SI - 4 Crystallographic Data Table 2

\begin{tabular}{|c|c|}
\hline Empirical formula & $\mathrm{C}_{9} \mathrm{H}_{10} \mathrm{O}_{2}$ \\
\hline Formula weight & $150.17 \mathrm{~g} / \mathrm{mol}$ \\
\hline Temperature & $173 \mathrm{~K}$ \\
\hline Wavelength & $1.54178 \AA$ \\
\hline Crystal system & monoclinic \\
\hline Space group & $\mathrm{P} 121 / \mathrm{c} 1$ \\
\hline Unit cell dimensions & $\begin{array}{l}\mathrm{a}=6.29510(10) \AA, \alpha=90^{\circ} \\
\mathrm{b}=10.34610(10) \AA, \beta=95.0990(10)^{\circ} \\
\mathrm{c}=10.97310(10) \AA, \gamma=90^{\circ}\end{array}$ \\
\hline Volume & $711.847(15) \AA^{3}$ \\
\hline $\mathbf{Z}$ & 4 \\
\hline Density (calculated) & $1.401 \mathrm{~g} / \mathrm{cm}^{3}$ \\
\hline Absorption coefficient & $0.800 \mathrm{~mm}^{-1}$ \\
\hline $\mathbf{F}(000)$ & 320 \\
\hline$\theta_{\max }$ & $70.03^{\circ}$ \\
\hline Crystal size $\left(\mathrm{mm}^{3}\right)$ & $0.118 \times 0.130 \times 0.397 \mathrm{~mm}$ \\
\hline Exposure time & $15.52 \mathrm{hr}$ \\
\hline $\begin{array}{l}\text { Reflections } \\
\text { collected }\end{array}$ & $\begin{array}{l}7232 \\
{\left[\mathrm{R}_{\text {int }}=1.54 \%\right]}\end{array}$ \\
\hline Independent reflections & 1318 \\
\hline Completeness to $\theta$ max & $97.3 \%$ \\
\hline Min./max. transmission & 0.7420 \\
\hline Refinement method & Full matrix least squares on F2 \\
\hline Absorption correction & Numerical \\
\hline Goodness-of-fit & 1.068 \\
\hline $\begin{array}{l}\text { Final } R \text { indices } \\
{[I>\mathbf{2 \theta}(\mathbf{I})]}\end{array}$ & $5.24 \%$ \\
\hline $\mathbf{R}$ indices (all ) & $10.31 \%$ \\
\hline $\begin{array}{l}\text { Largest diff. peak } \\
\text { and hole }\end{array}$ & $0.278,-0.251 \mathrm{e}^{-} / \AA^{3}$ \\
\hline
\end{tabular}

Figure SI - 9 X-Ray crystal structure. Atomic displacement ellipsoids generated at $50 \%$ probability Recrystallized from a minimum of hot hexanes.
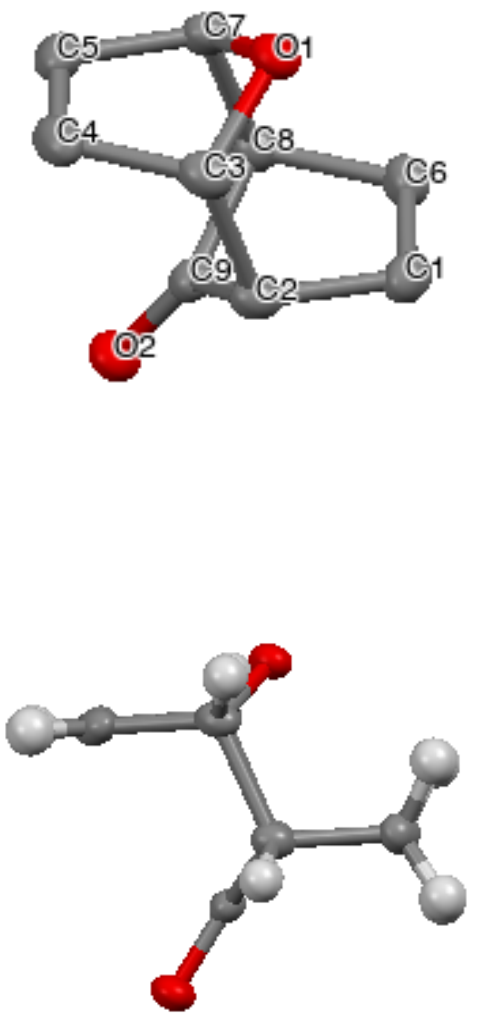
Table SI - 5 Dihedral Angles $\left(^{\circ}\right) 2$

\begin{tabular}{|c|c|c|c|c|}
\hline Number & Atom1 & Atom2 & Atom3 & Angle \\
\hline 1 & C3 & O1 & $\mathrm{C} 7$ & $101.44(9)$ \\
\hline 2 & $\mathrm{H} 1$ & $\mathrm{C} 1$ & H9 & 108.8 \\
\hline 3 & H1 & $\mathrm{C} 1$ & $\mathrm{C} 2$ & 110.7 \\
\hline 4 & $\mathrm{H} 1$ & $\mathrm{C} 1$ & C6 & 110.7 \\
\hline 5 & H9 & $\mathrm{C} 1$ & $\mathrm{C} 2$ & 110.7 \\
\hline 6 & H9 & $\mathrm{C} 1$ & C6 & 110.7 \\
\hline 7 & $\mathrm{C} 2$ & $\mathrm{C} 1$ & C6 & 105.2(1) \\
\hline 8 & $\mathrm{C} 1$ & $\mathrm{C} 2$ & H8 & 113.5 \\
\hline 9 & $\mathrm{C} 1$ & $\mathrm{C} 2$ & $\mathrm{C} 3$ & $109.4(1)$ \\
\hline 10 & $\mathrm{C} 1$ & $\mathrm{C} 2$ & C9 & $100.0(1)$ \\
\hline 11 & $\mathrm{H} 8$ & $\mathrm{C} 2$ & $\mathrm{C} 3$ & 113.5 \\
\hline 12 & $\mathrm{H} 8$ & $\mathrm{C} 2$ & C9 & 113.6 \\
\hline 13 & $\mathrm{C} 3$ & $\mathrm{C} 2$ & C9 & $105.7(1)$ \\
\hline 14 & $\mathrm{O} 1$ & $\mathrm{C} 3$ & $\mathrm{C} 2$ & $106.8(1)$ \\
\hline 15 & $\mathrm{O} 1$ & $\mathrm{C} 3$ & $\mathrm{H} 7$ & 112.2 \\
\hline 16 & $\mathrm{O} 1$ & $\mathrm{C} 3$ & $\mathrm{C} 4$ & $101.7(1)$ \\
\hline 17 & $\mathrm{C} 2$ & $\mathrm{C} 3$ & $\mathrm{H} 7$ & 112.2 \\
\hline 18 & $\mathrm{C} 2$ & $\mathrm{C} 3$ & $\mathrm{C} 4$ & 111.1(1) \\
\hline 19 & $\mathrm{H} 7$ & $\mathrm{C} 3$ & $\mathrm{C} 4$ & 112.2 \\
\hline 20 & C3 & $\mathrm{C} 4$ & H5 & 126.3 \\
\hline 21 & $\mathrm{C} 3$ & $\mathrm{C} 4$ & C5 & 107.2(1) \\
\hline 22 & H5 & $\mathrm{C} 4$ & C5 & 126.4 \\
\hline 23 & $\mathrm{C} 4$ & C5 & $\mathrm{H} 2$ & 126.3 \\
\hline 24 & $\mathrm{C} 4$ & C5 & C7 & $107.3(1)$ \\
\hline 25 & $\mathrm{H} 2$ & C5 & $\mathrm{C} 7$ & 126.3 \\
\hline 26 & $\mathrm{C} 1$ & C6 & $\mathrm{H} 10$ & 110.7 \\
\hline 27 & $\mathrm{C} 1$ & C6 & $\mathrm{H} 3$ & 110.7 \\
\hline 28 & $\mathrm{C} 1$ & C6 & $\mathrm{C} 8$ & $105.2(1)$ \\
\hline 29 & H10 & C6 & H3 & 108.8 \\
\hline 30 & $\mathrm{H} 10$ & C6 & C8 & 110.7 \\
\hline 31 & $\mathrm{H} 3$ & C6 & $\mathrm{C} 8$ & 110.7 \\
\hline 32 & $\mathrm{O} 1$ & $\mathrm{C} 7$ & C5 & 101.5(1) \\
\hline 33 & $\mathrm{O} 1$ & $\mathrm{C} 7$ & H6 & 112.4 \\
\hline 34 & $\mathrm{O} 1$ & $\mathrm{C} 7$ & C8 & 107.3(1) \\
\hline 35 & C5 & C7 & H6 & 112.4 \\
\hline 36 & C5 & $\mathrm{C} 7$ & C8 & 110.1(1) \\
\hline 37 & H6 & $\mathrm{C} 7$ & $\mathrm{C} 8$ & 112.4 \\
\hline 38 & C6 & $\mathrm{C} 8$ & C7 & 109.5(1) \\
\hline 39 & C6 & C8 & $\mathrm{H} 4$ & 113.5 \\
\hline
\end{tabular}




\begin{tabular}{|r|l|l|l|r|}
\hline $\mathbf{4 0}$ & C6 & C8 & C9 & $99.5(1)$ \\
\hline $\mathbf{4 1}$ & C7 & C8 & H4 & 113.5 \\
\hline $\mathbf{4 2}$ & C7 & C8 & C9 & $106.2(1)$ \\
\hline $\mathbf{4 3}$ & H4 & C8 & C9 & 113.5 \\
\hline $\mathbf{4 4}$ & O2 & C9 & C2 & $128.2(1)$ \\
\hline $\mathbf{4 5}$ & O2 & C9 & C8 & $128.5(1)$ \\
\hline $\mathbf{4 6}$ & C2 & C9 & C8 & $103.1(1)$ \\
\hline
\end{tabular}


Table SI - 6 Bond Length (§) 2

\begin{tabular}{|c|c|c|c|}
\hline Number & Atom1 & Atom2 & Length \\
\hline 1 & $\mathrm{O} 1$ & $\mathrm{C} 3$ & $1.433(2)$ \\
\hline 2 & $\mathrm{O} 1$ & $\mathrm{C} 7$ & $1.436(2)$ \\
\hline 3 & $\mathrm{O} 2$ & C9 & $1.210(2)$ \\
\hline 4 & $\mathrm{C} 1$ & H1 & 0.989 \\
\hline 5 & $\mathrm{C} 1$ & H9 & 0.990 \\
\hline 6 & $\mathrm{C} 1$ & $\mathrm{C} 2$ & $1.544(2)$ \\
\hline 7 & $\mathrm{C} 1$ & C6 & $1.565(2)$ \\
\hline 8 & $\mathrm{C} 2$ & $\mathrm{H} 8$ & 1.000 \\
\hline 9 & $\mathrm{C} 2$ & $\mathrm{C} 3$ & $1.570(2)$ \\
\hline 10 & $\mathrm{C} 2$ & C9 & $1.517(2)$ \\
\hline 11 & $\mathrm{C} 3$ & $\mathrm{H} 7$ & 1.000 \\
\hline 12 & $\mathrm{C} 3$ & $\mathrm{C} 4$ & $1.514(2)$ \\
\hline 13 & $\mathrm{C} 4$ & H5 & 0.950 \\
\hline 14 & $\mathrm{C} 4$ & $\mathrm{C} 5$ & $1.321(2)$ \\
\hline 15 & $\mathrm{C} 5$ & $\mathrm{H} 2$ & 0.950 \\
\hline 16 & $\mathrm{C} 5$ & $\mathrm{C} 7$ & $1.515(2)$ \\
\hline 17 & C6 & $\mathrm{H} 10$ & 0.990 \\
\hline 18 & C6 & $\mathrm{H} 3$ & 0.990 \\
\hline 19 & C6 & $\mathrm{C} 8$ & $1.545(2)$ \\
\hline 20 & $\mathrm{C} 7$ & H6 & 1.000 \\
\hline 21 & $\mathrm{C} 7$ & $\mathrm{C} 8$ & $1.573(2)$ \\
\hline 22 & $\mathrm{C} 8$ & $\mathrm{H} 4$ & 1.000 \\
\hline 23 & $\mathrm{C} 8$ & C9 & $1.515(2)$ \\
\hline
\end{tabular}


ชัญ

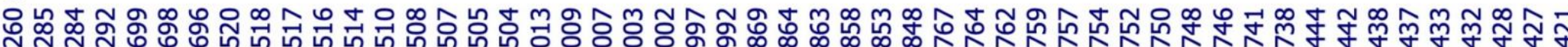
.

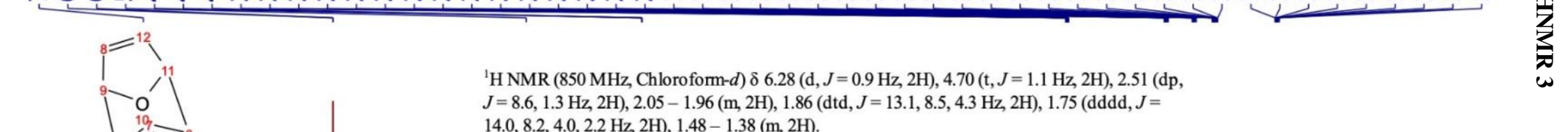




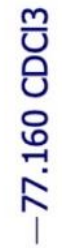

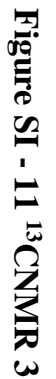
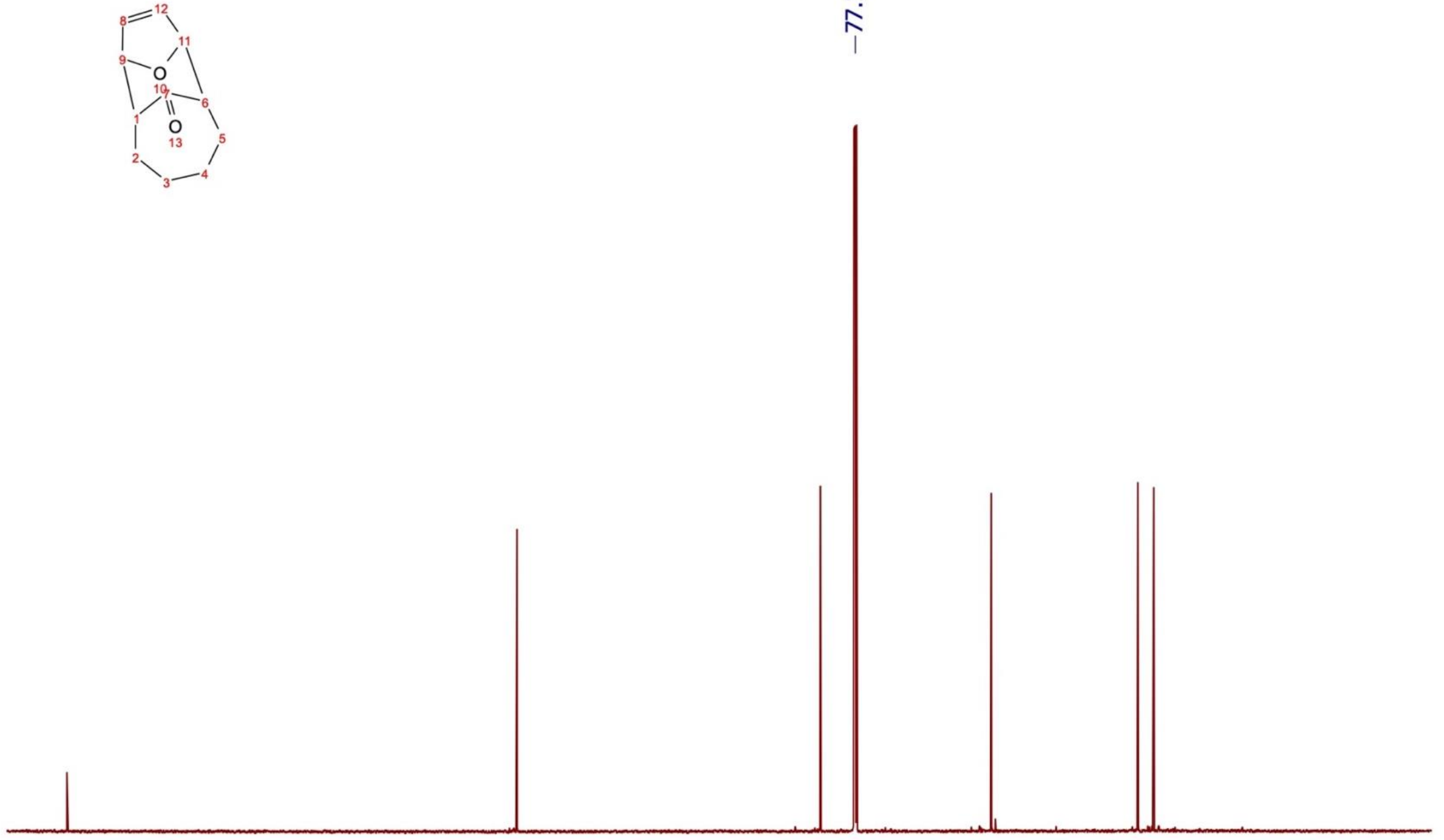

\begin{tabular}{llllllllllllllllllllllllll}
\hline 20 & 210 & 200 & 190 & 180 & 170 & 160 & 150 & 140 & 130 & 120 & 110 & 100 & 90 & 80 & 70 & 60 & 50 & 40 & 30 & 20 & 10 & 0 & -10 &
\end{tabular} 


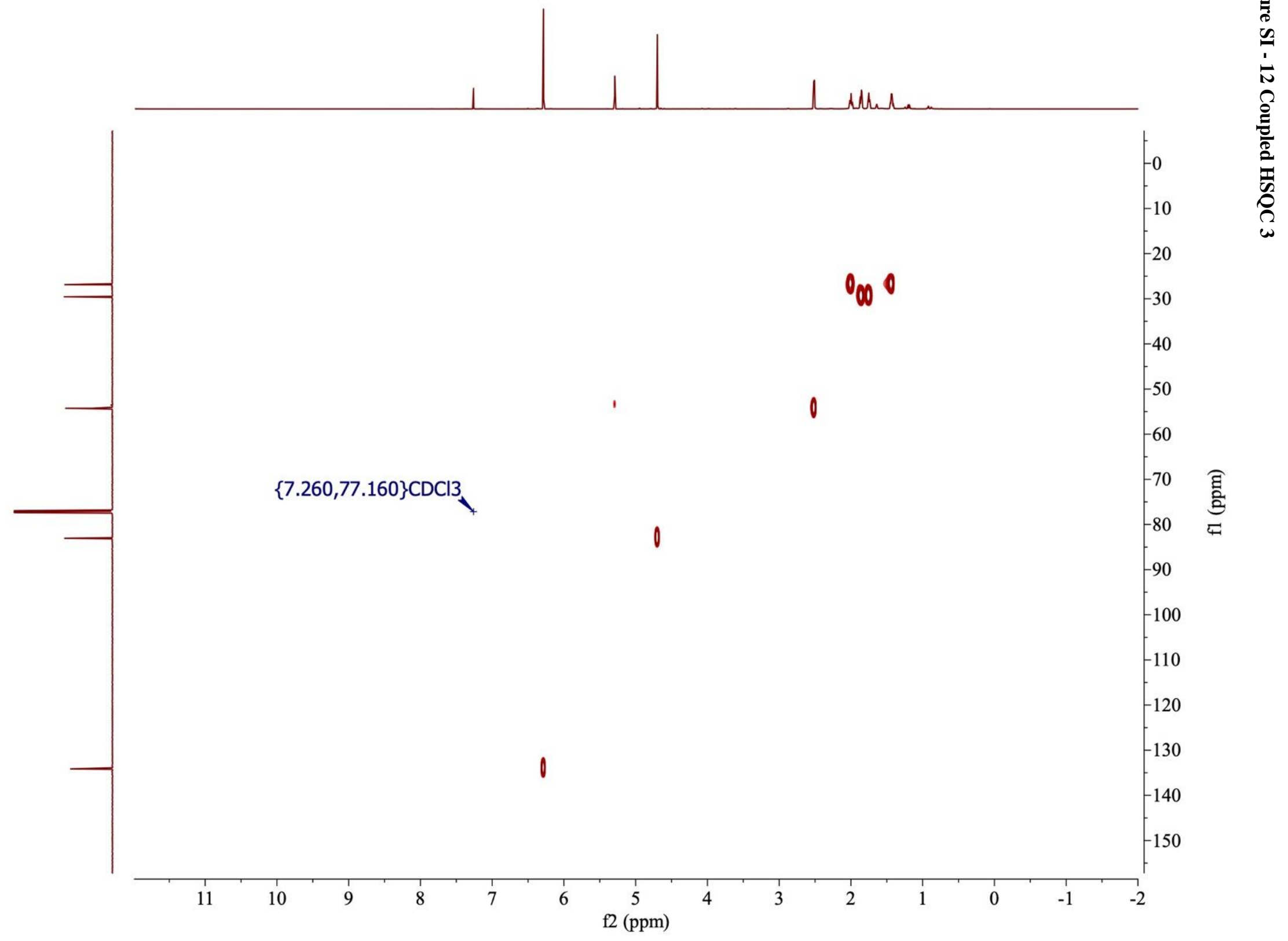




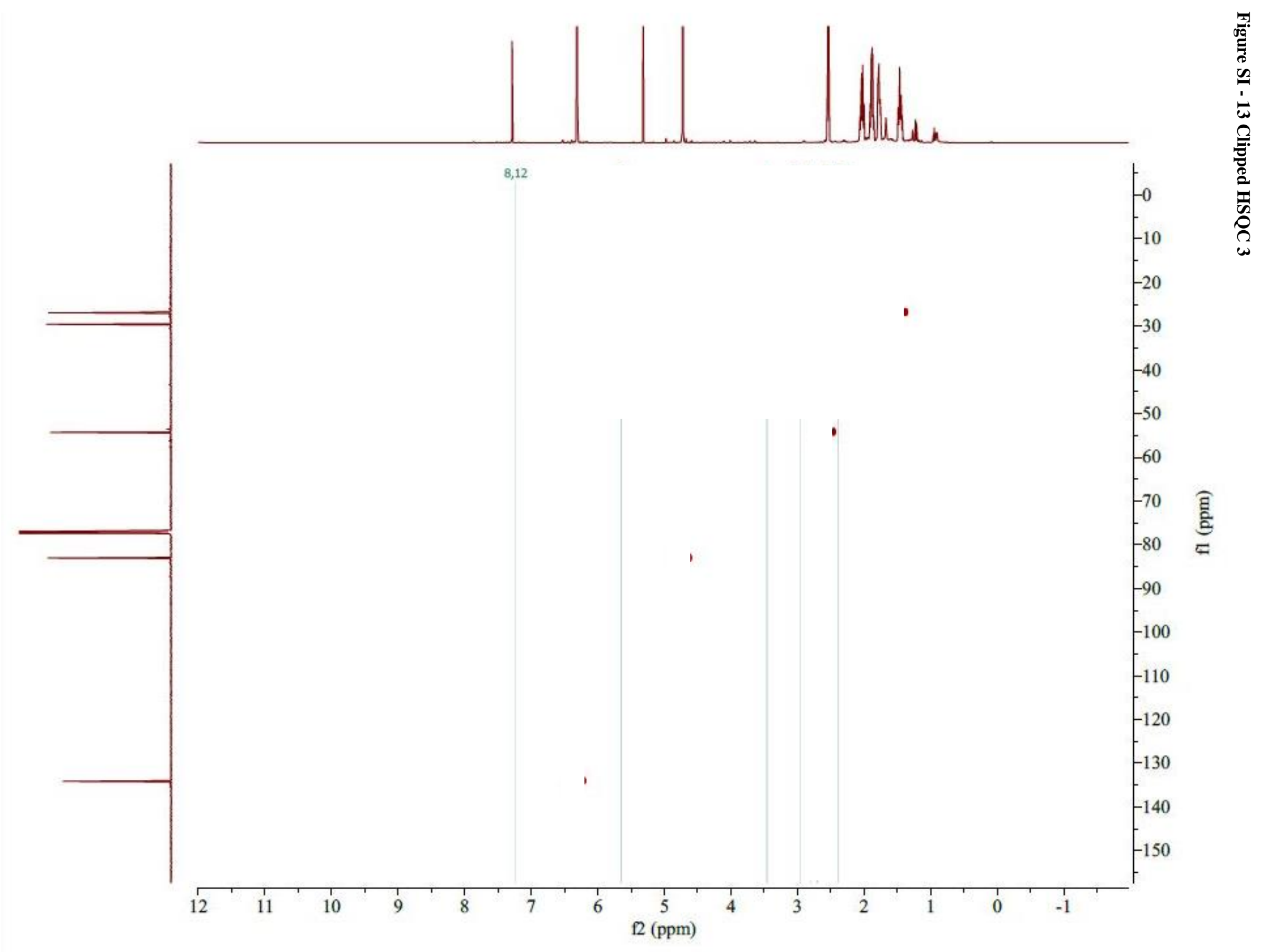




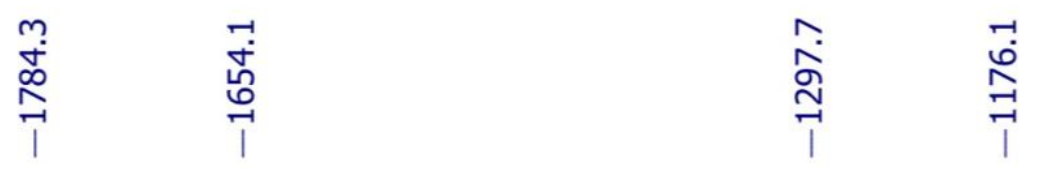

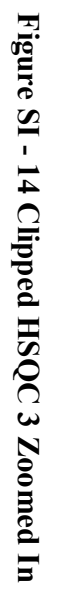

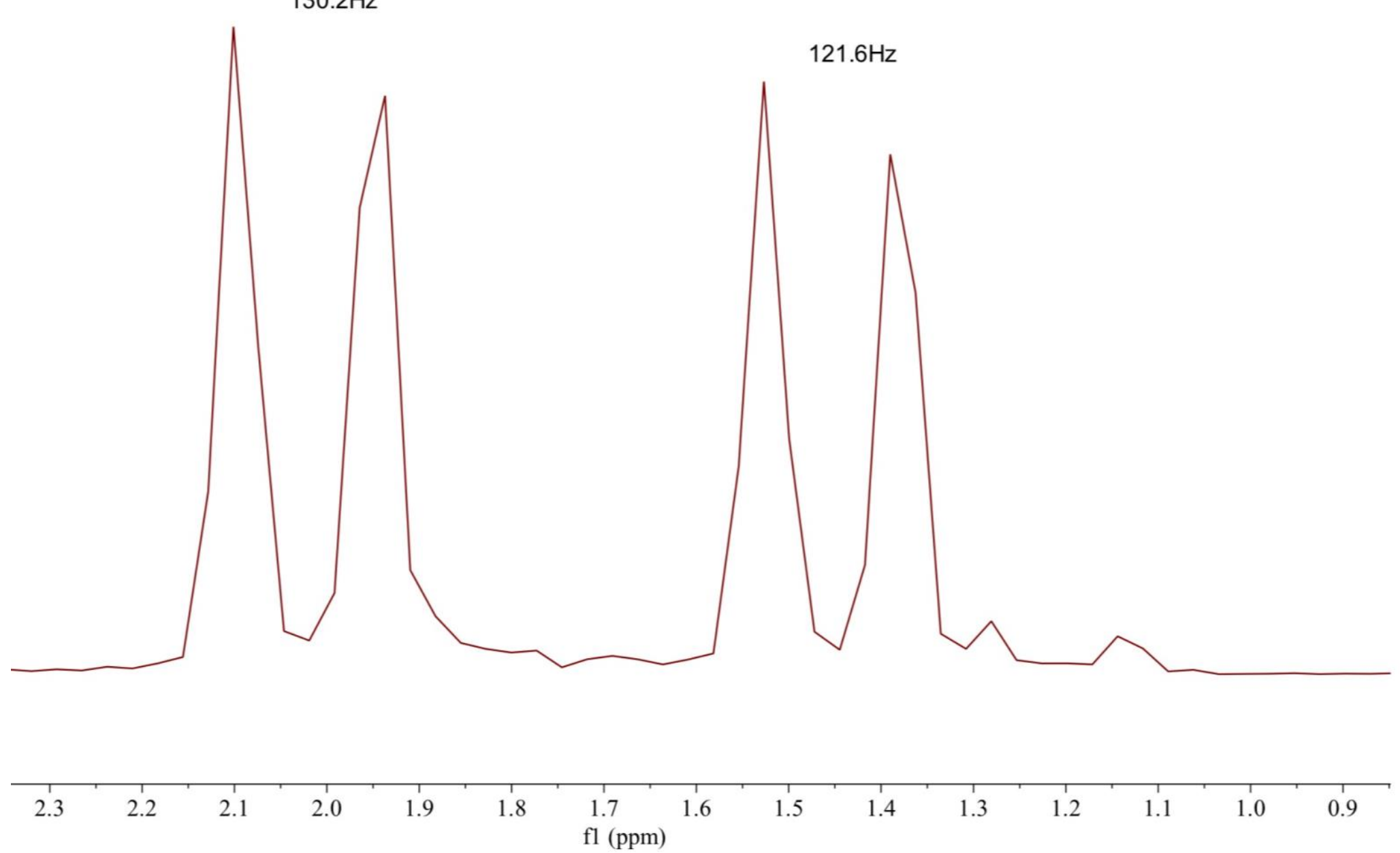




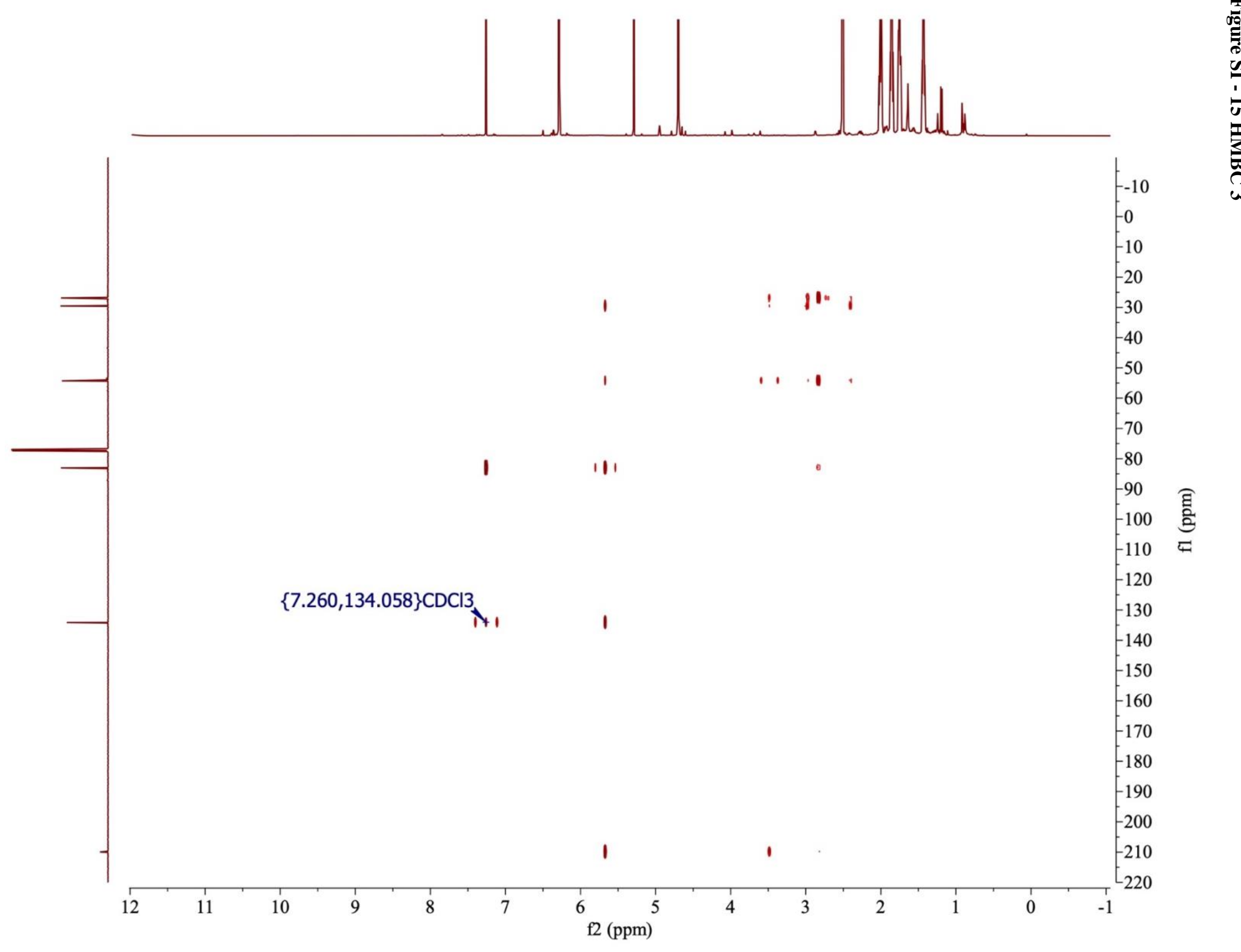

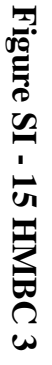




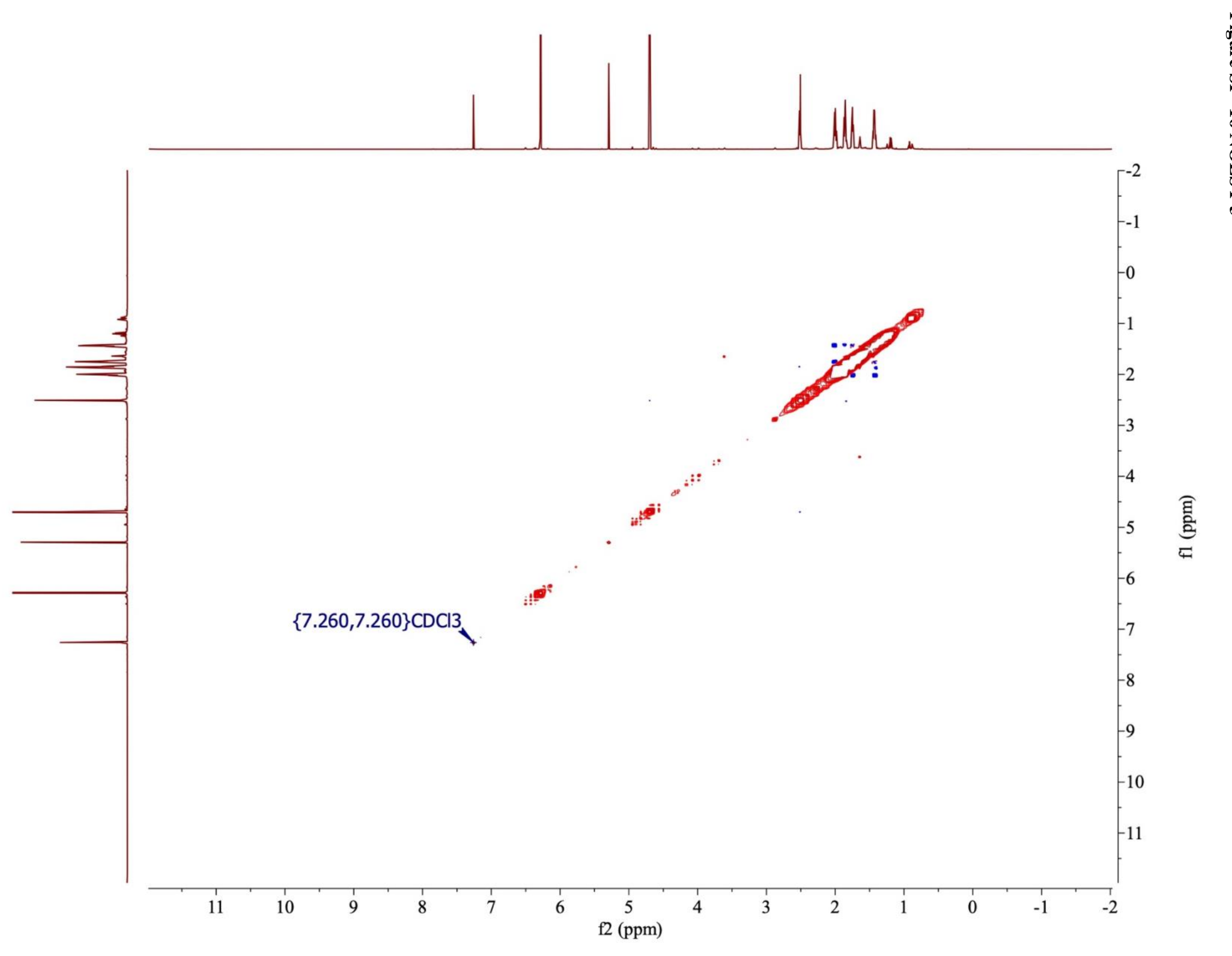

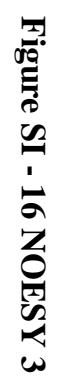




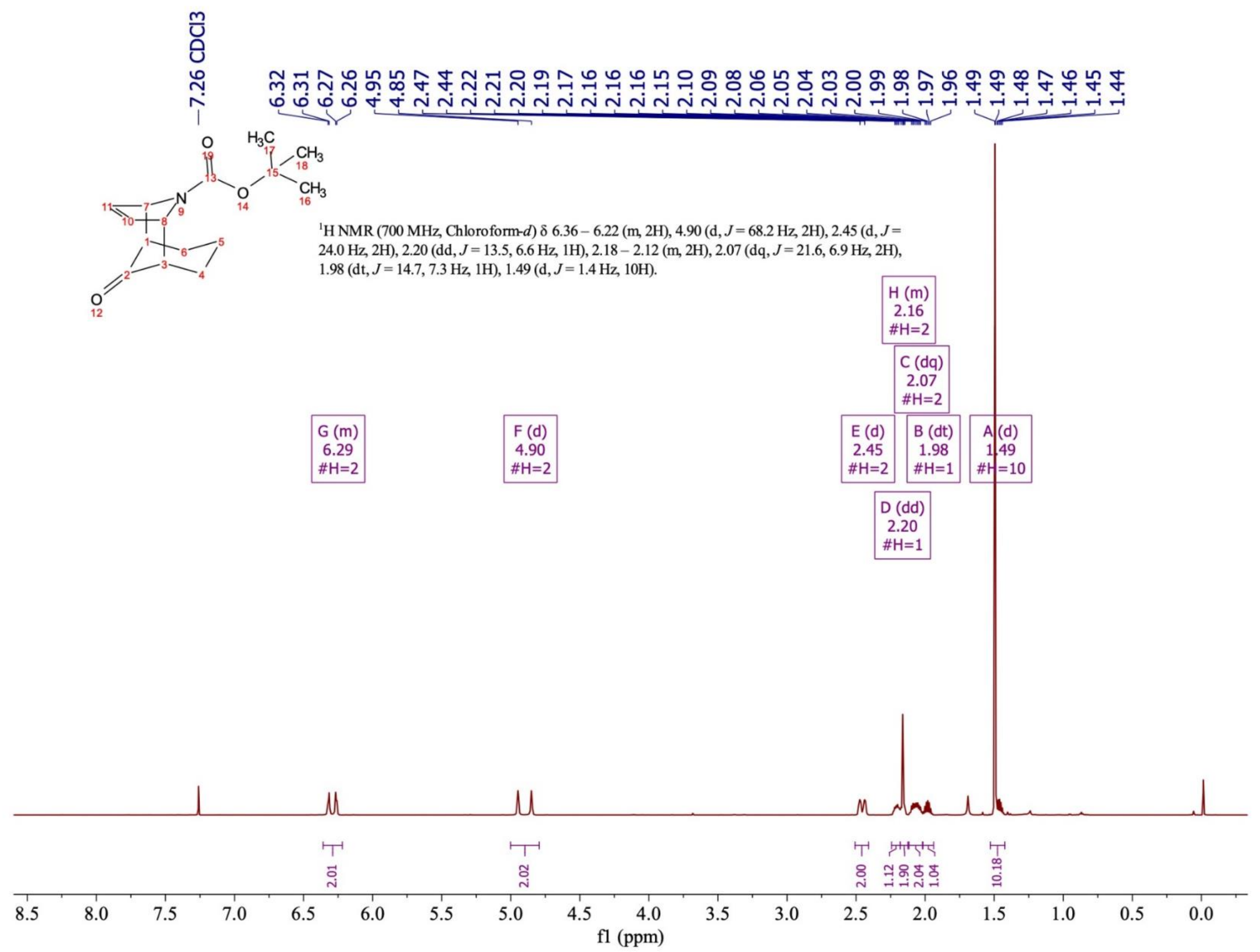



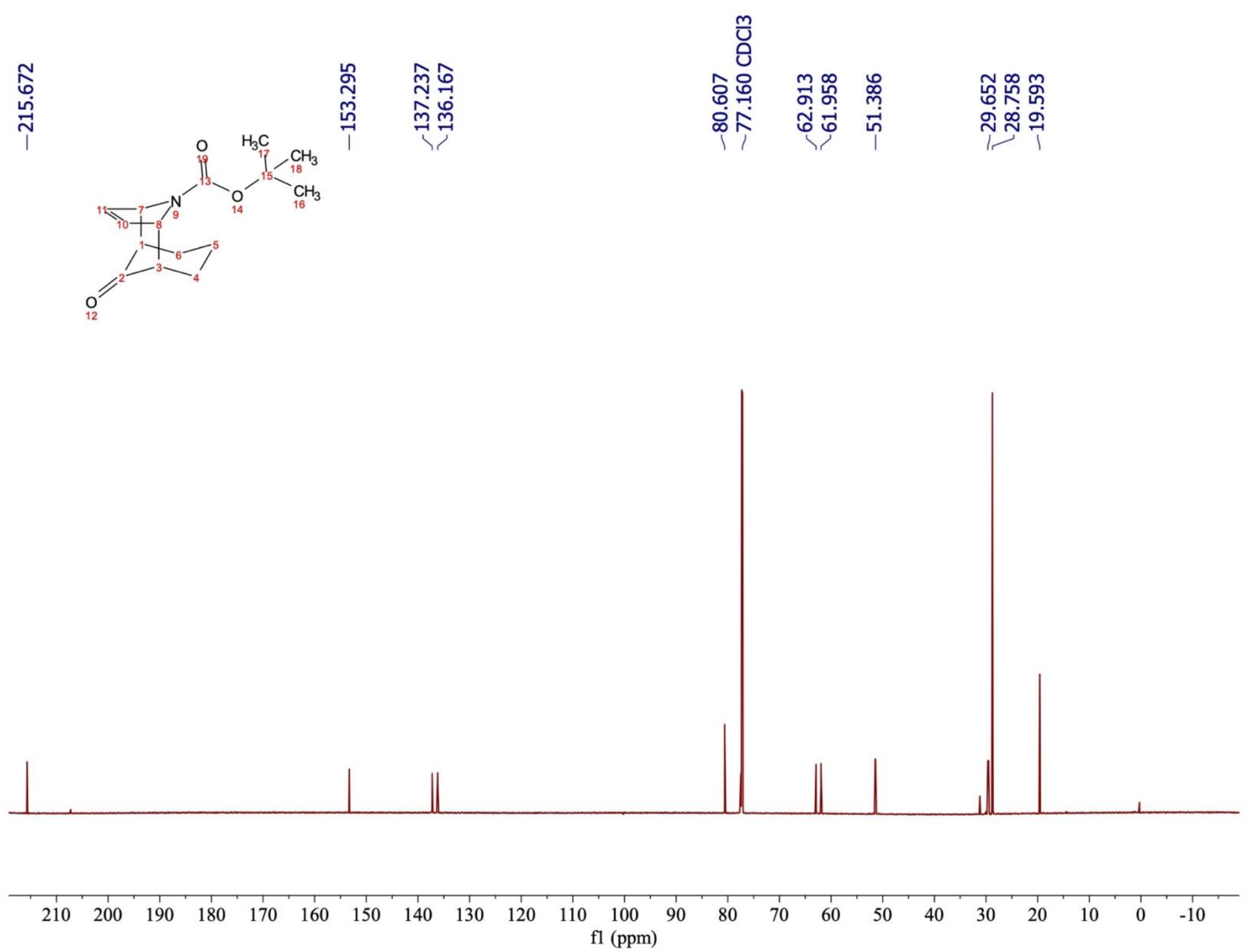


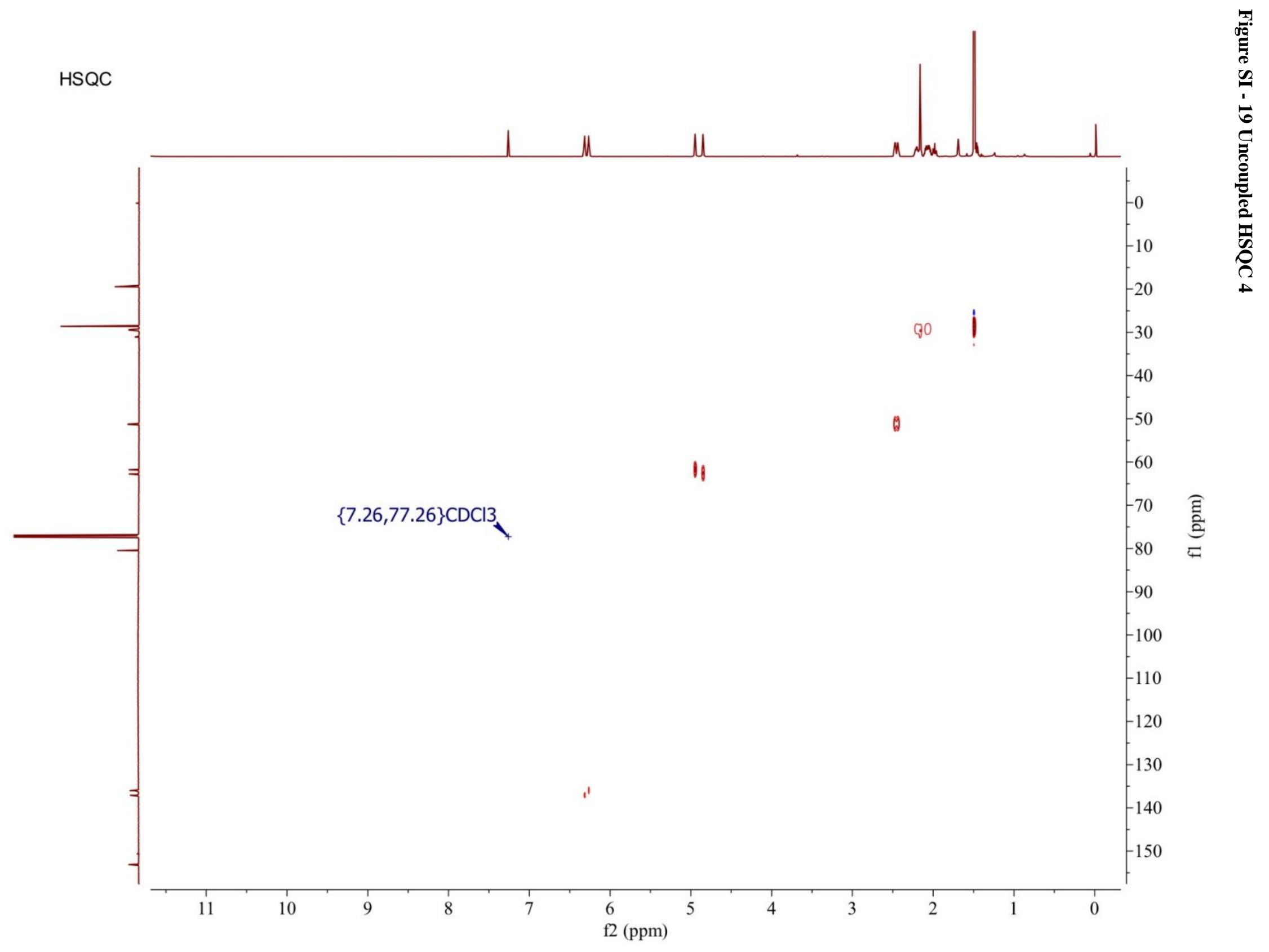




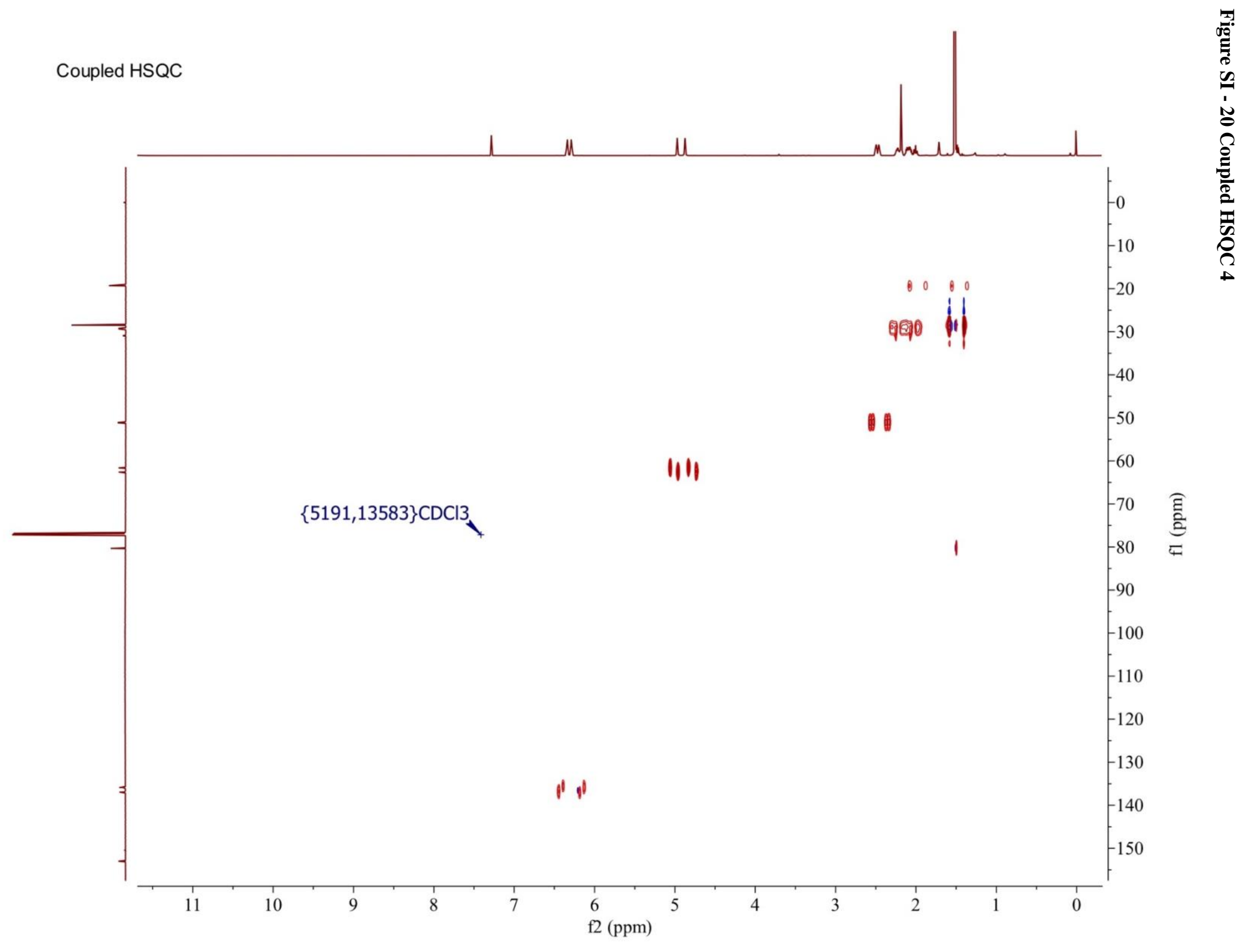




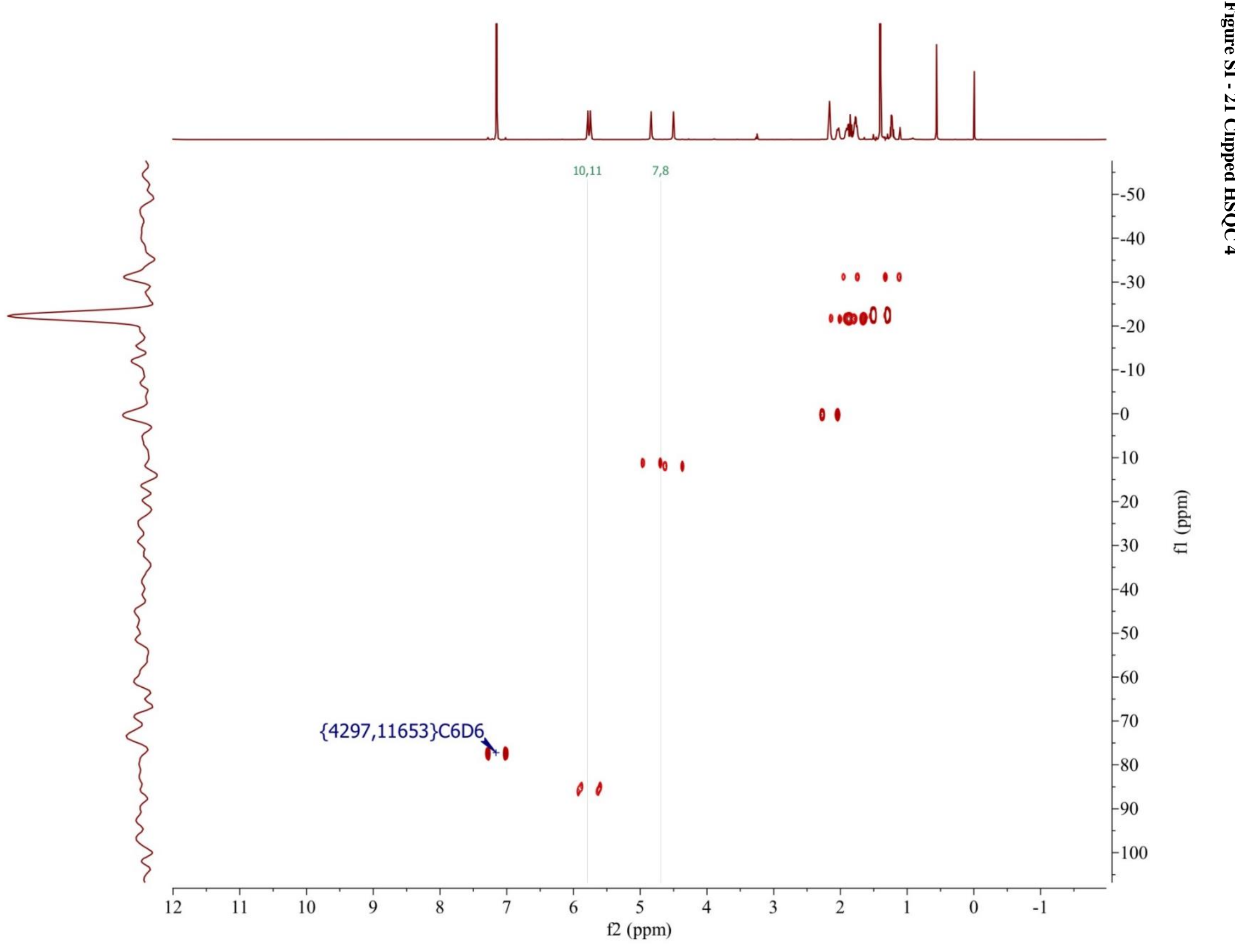

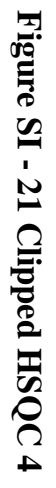




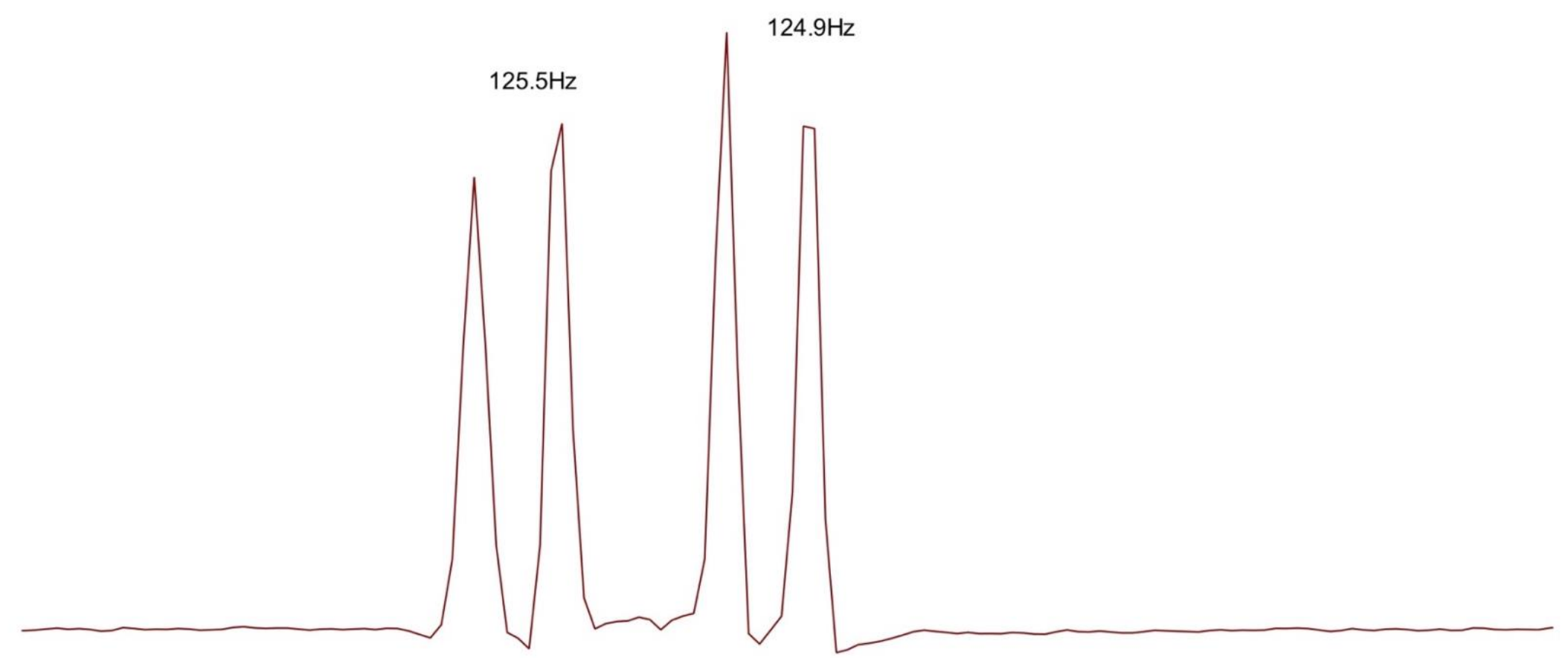

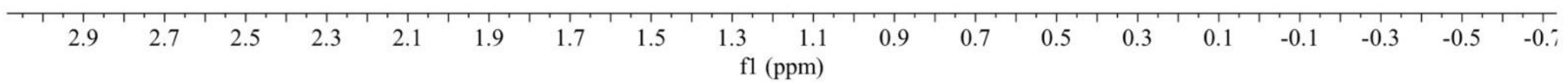




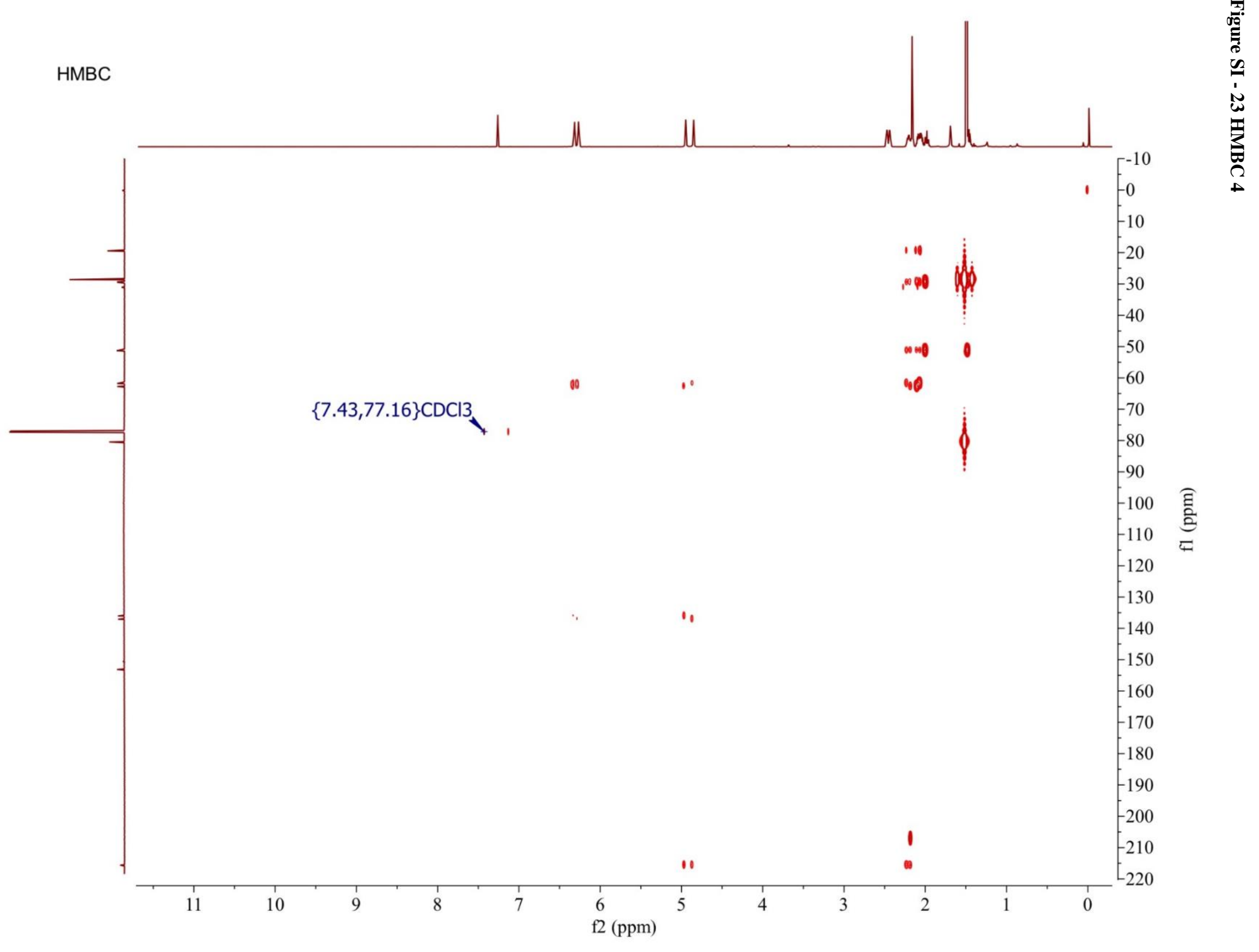




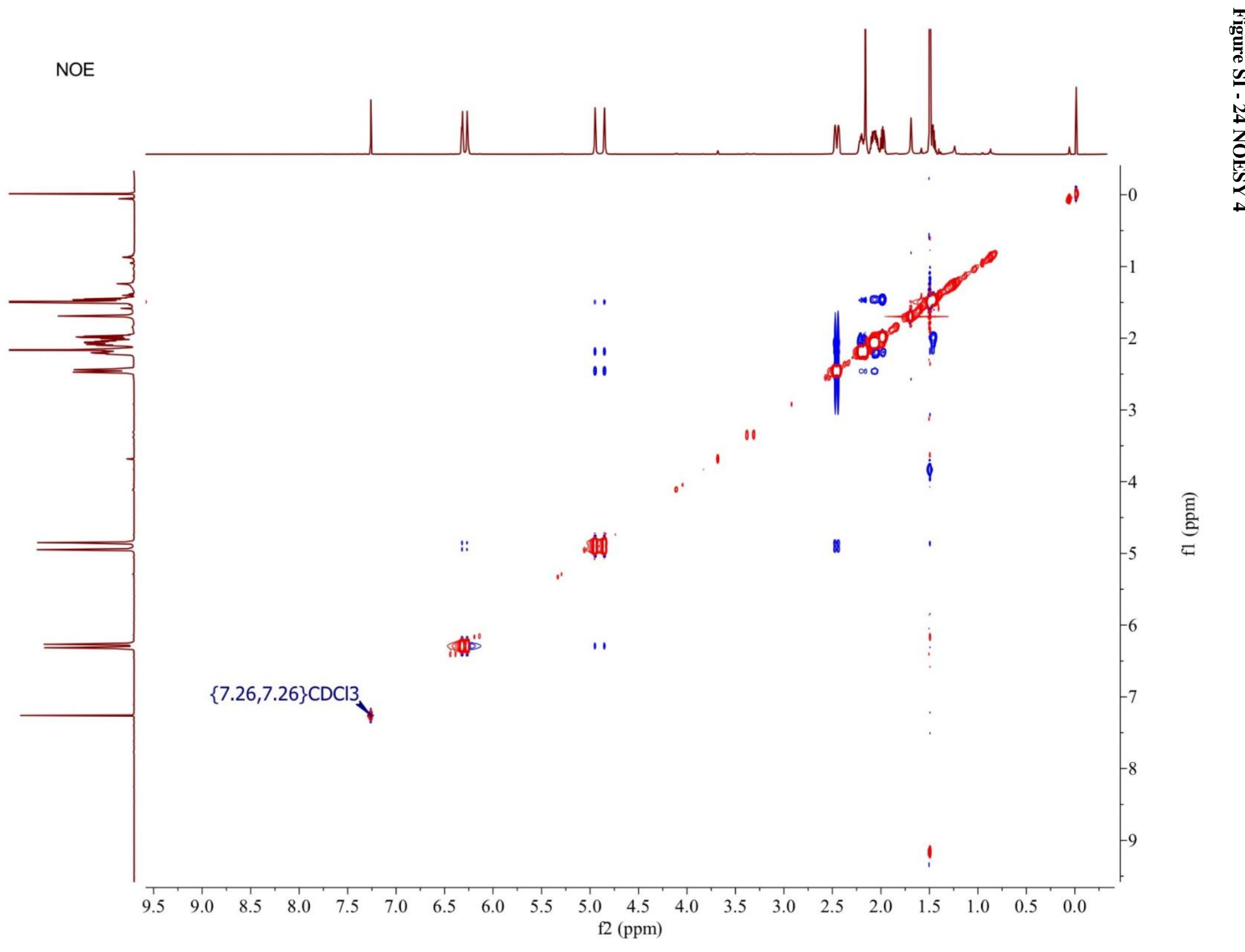



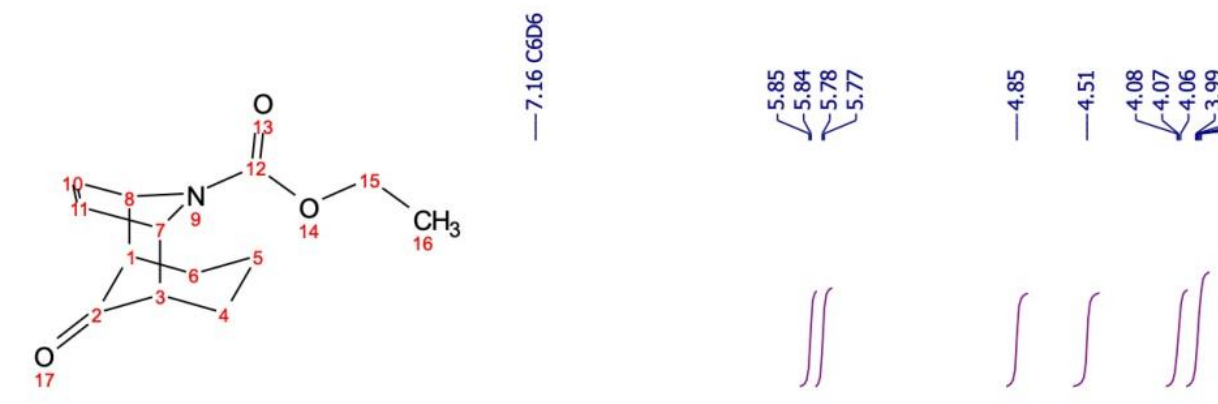

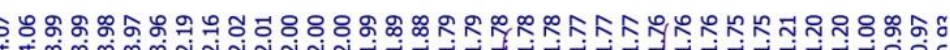

${ }^{1} \mathrm{H}$ NMR (700 MHz, Chloroform- $d$ ) $\delta 5.84(\mathrm{~d}, J=6.0 \mathrm{~Hz}, 1 \mathrm{H}), 5.78(\mathrm{~d}, J=6.1 \mathrm{~Hz}, 1 \mathrm{H}), 4.85$ (s, $1 \mathrm{H}), 4.51(\mathrm{~s}, 1 \mathrm{H}), 4.08(\mathrm{dd}, J=10.0,6.3 \mathrm{~Hz}, 1 \mathrm{H}), 4.03-3.93(\mathrm{~m}, 1 \mathrm{H}), 2.18(\mathrm{~d}, J=21.5 \mathrm{~Hz}, 2 \mathrm{H})$,

$2.09-1.95(\mathrm{~m}, 1 \mathrm{H}), 1.95-1.83(\mathrm{~m}, 1 \mathrm{H}), 1.77$ (tdd, $J=11.5,7.4,5.4 \mathrm{~Hz}, 3 \mathrm{H}), 1.26-1.14(\mathrm{~m}$,

$1 \mathrm{H}), 0.99(\mathrm{t}, J=7.1 \mathrm{~Hz}, 3 \mathrm{H})$

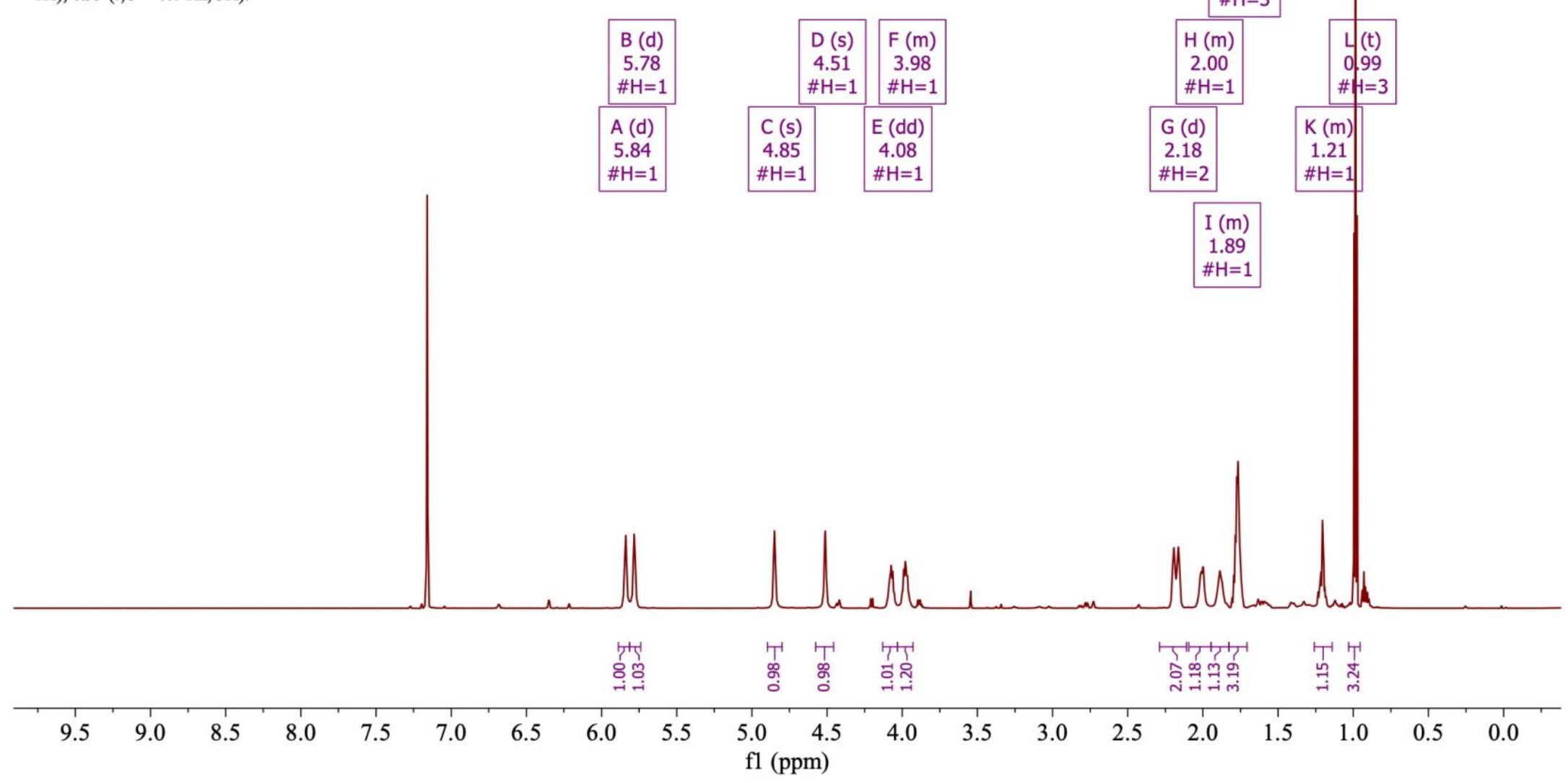



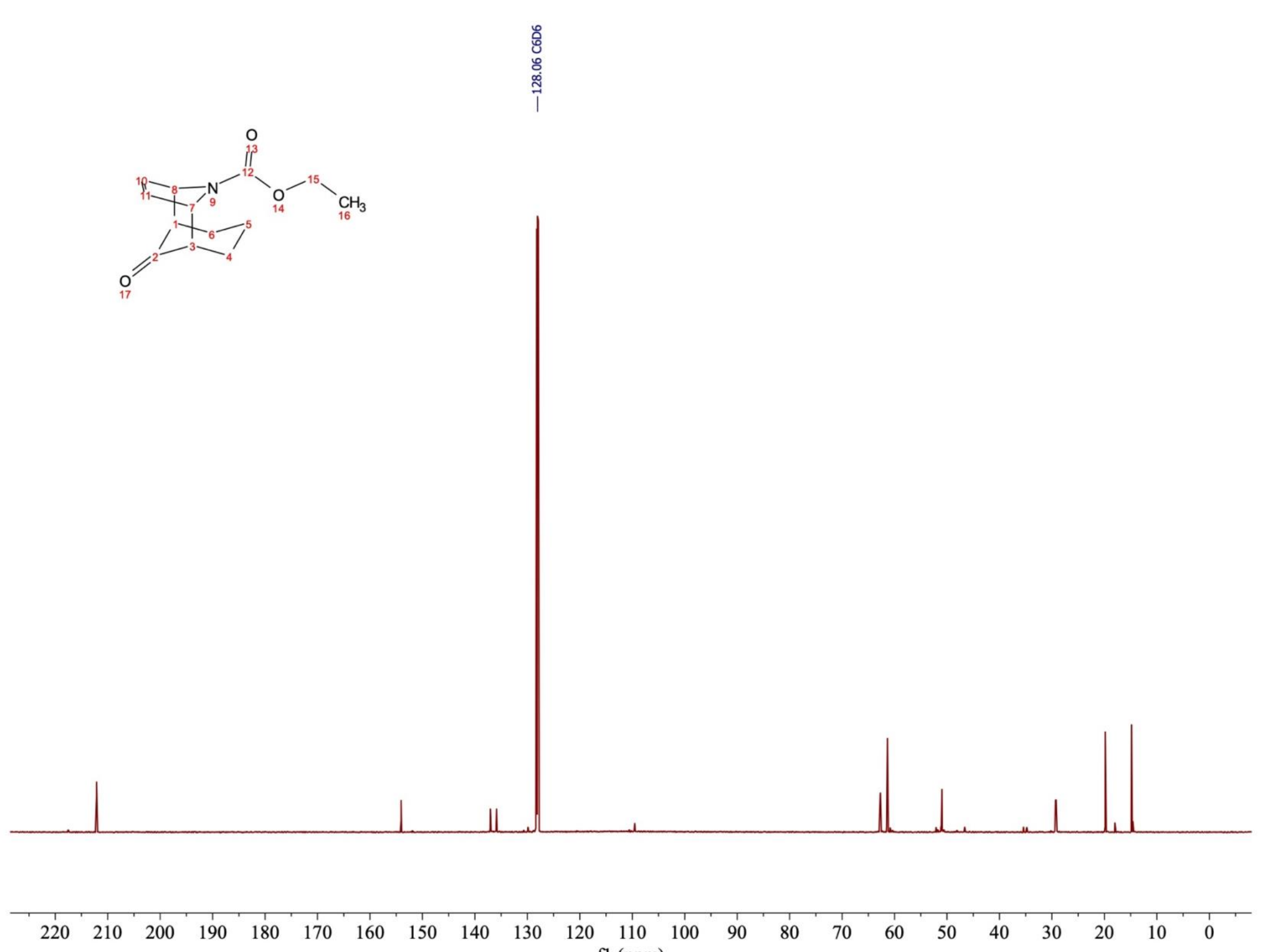


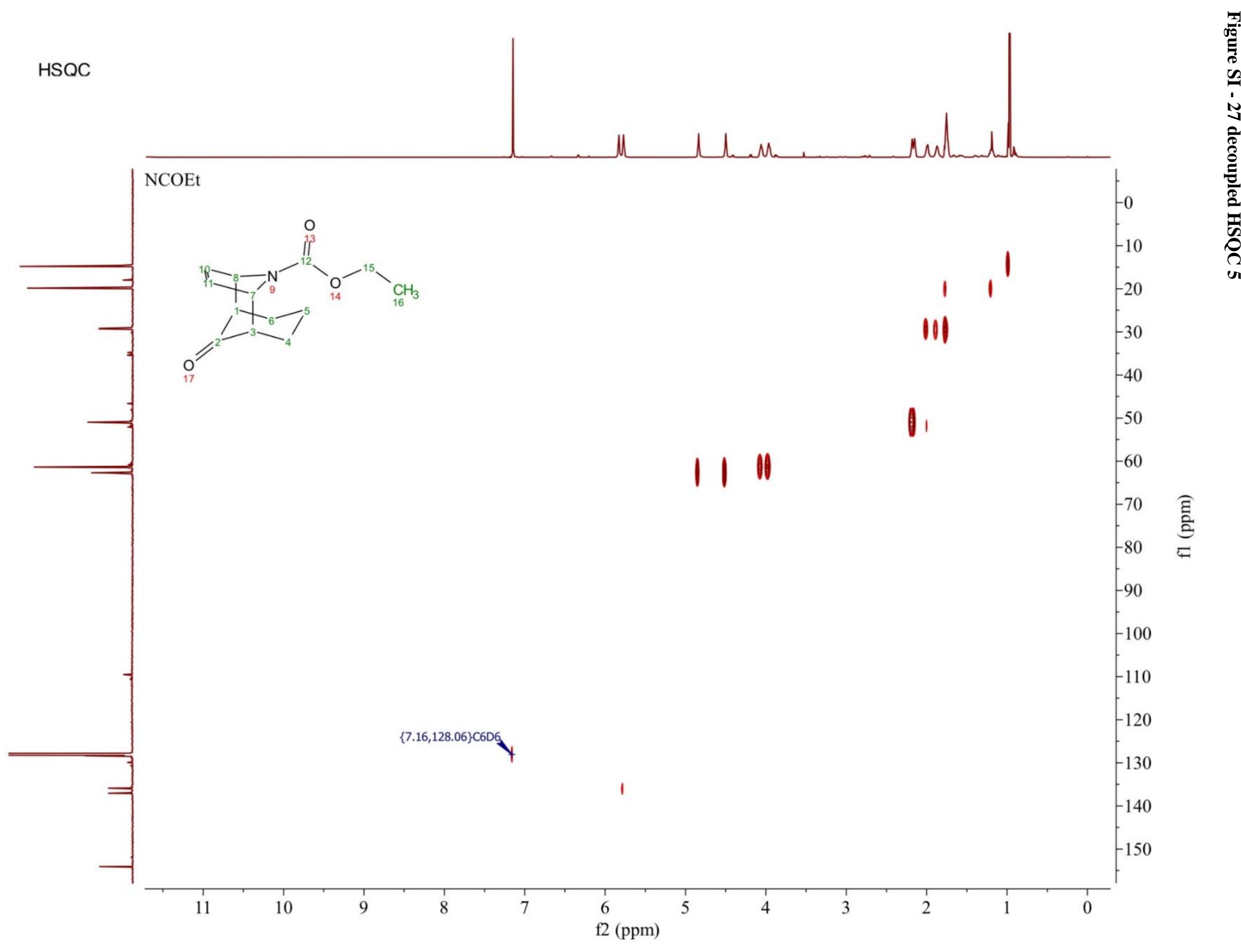




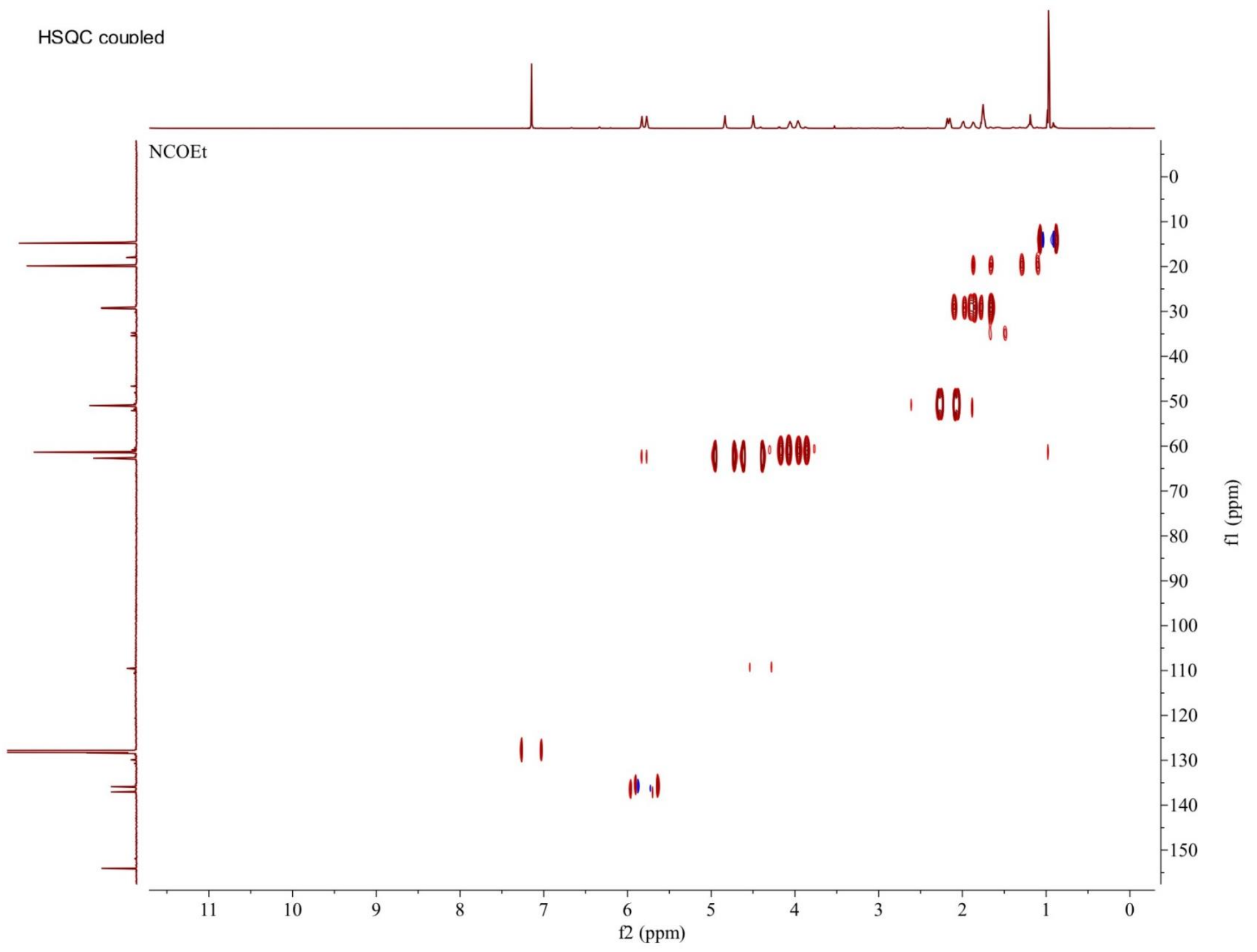




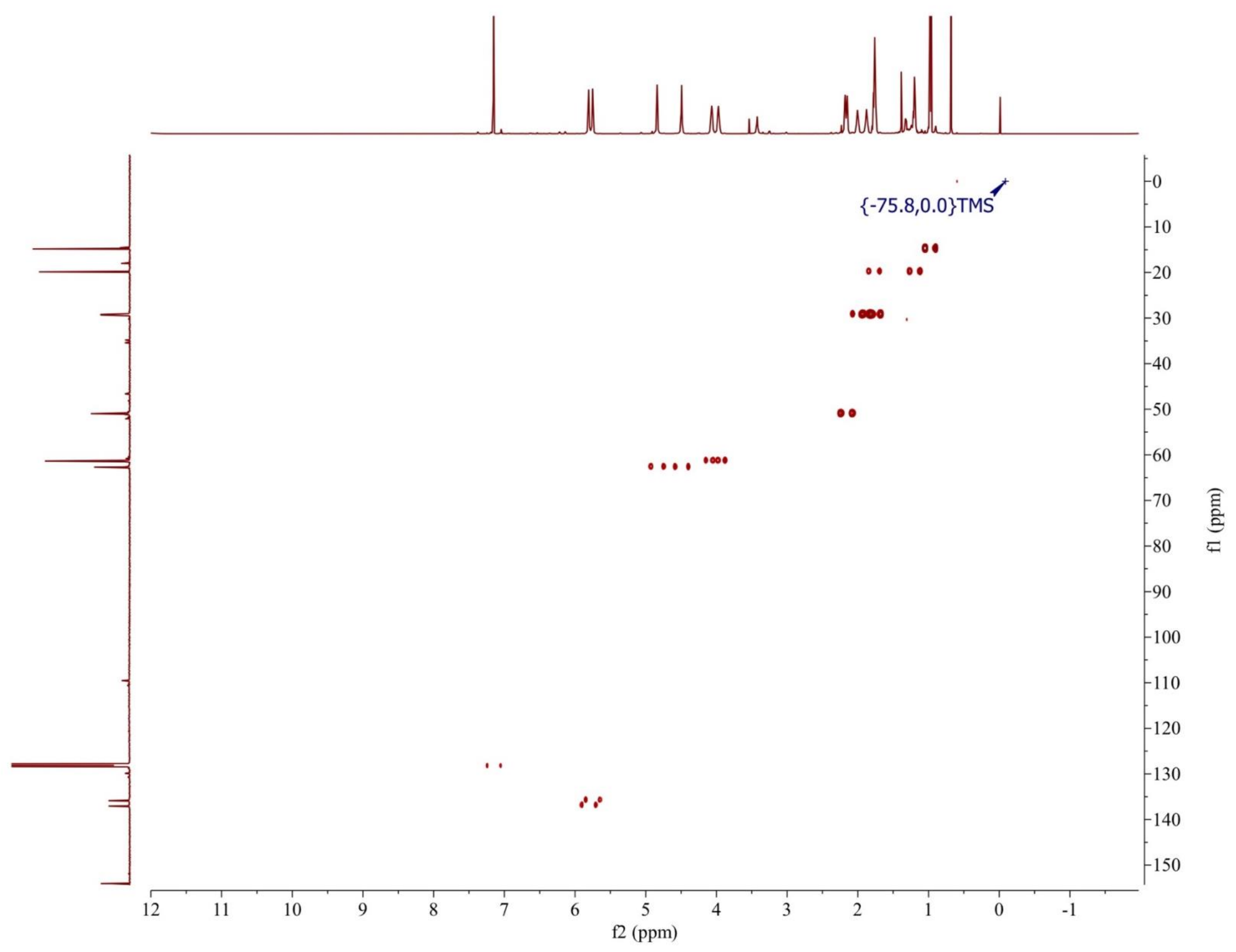




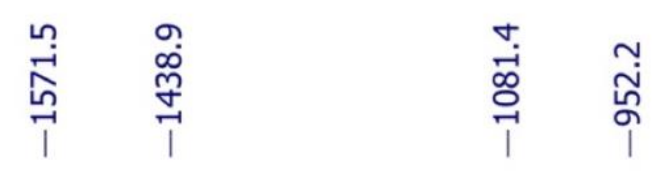

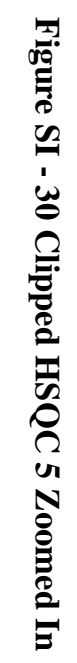

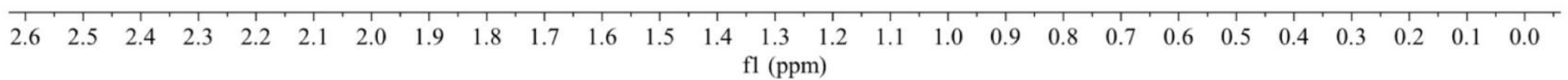




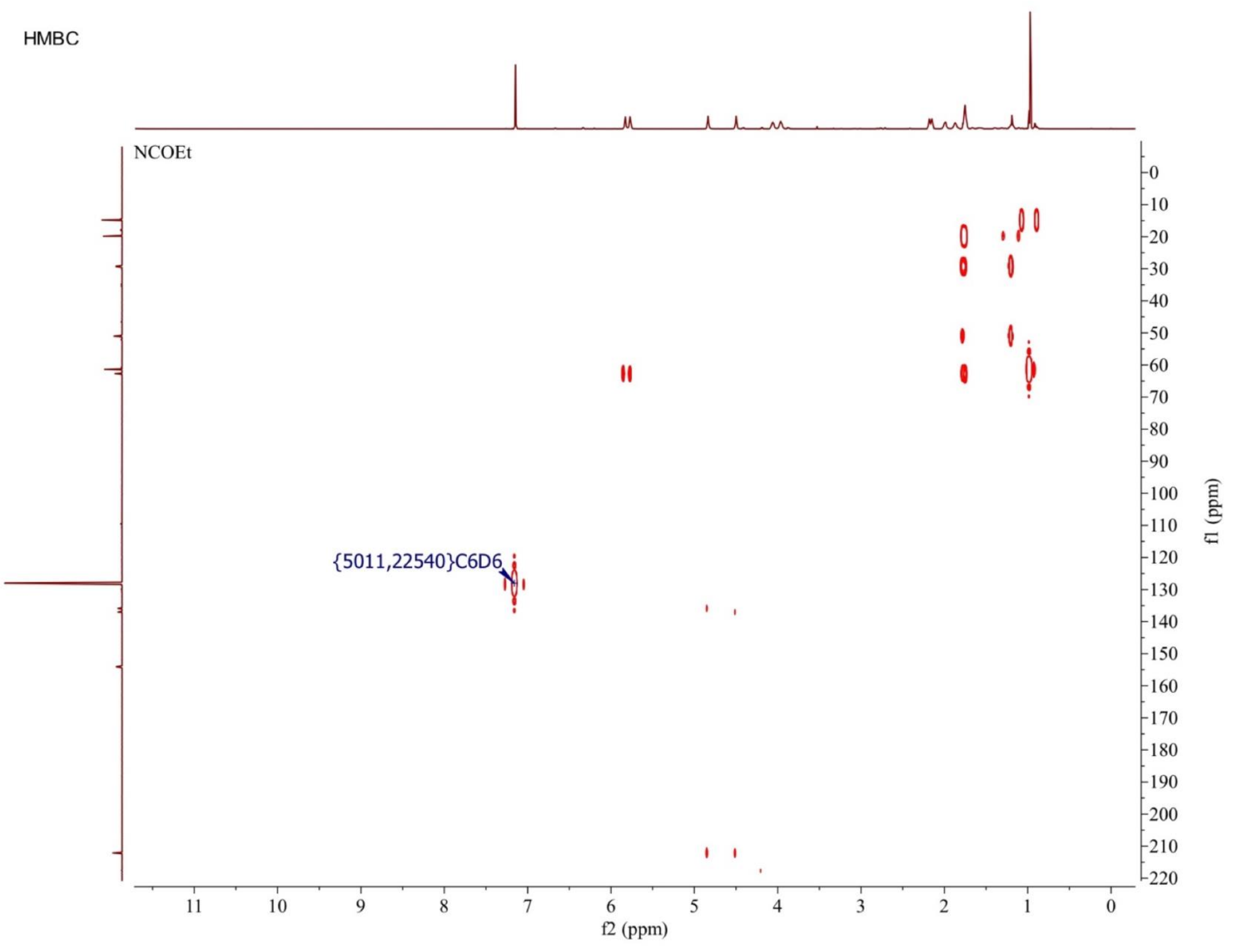




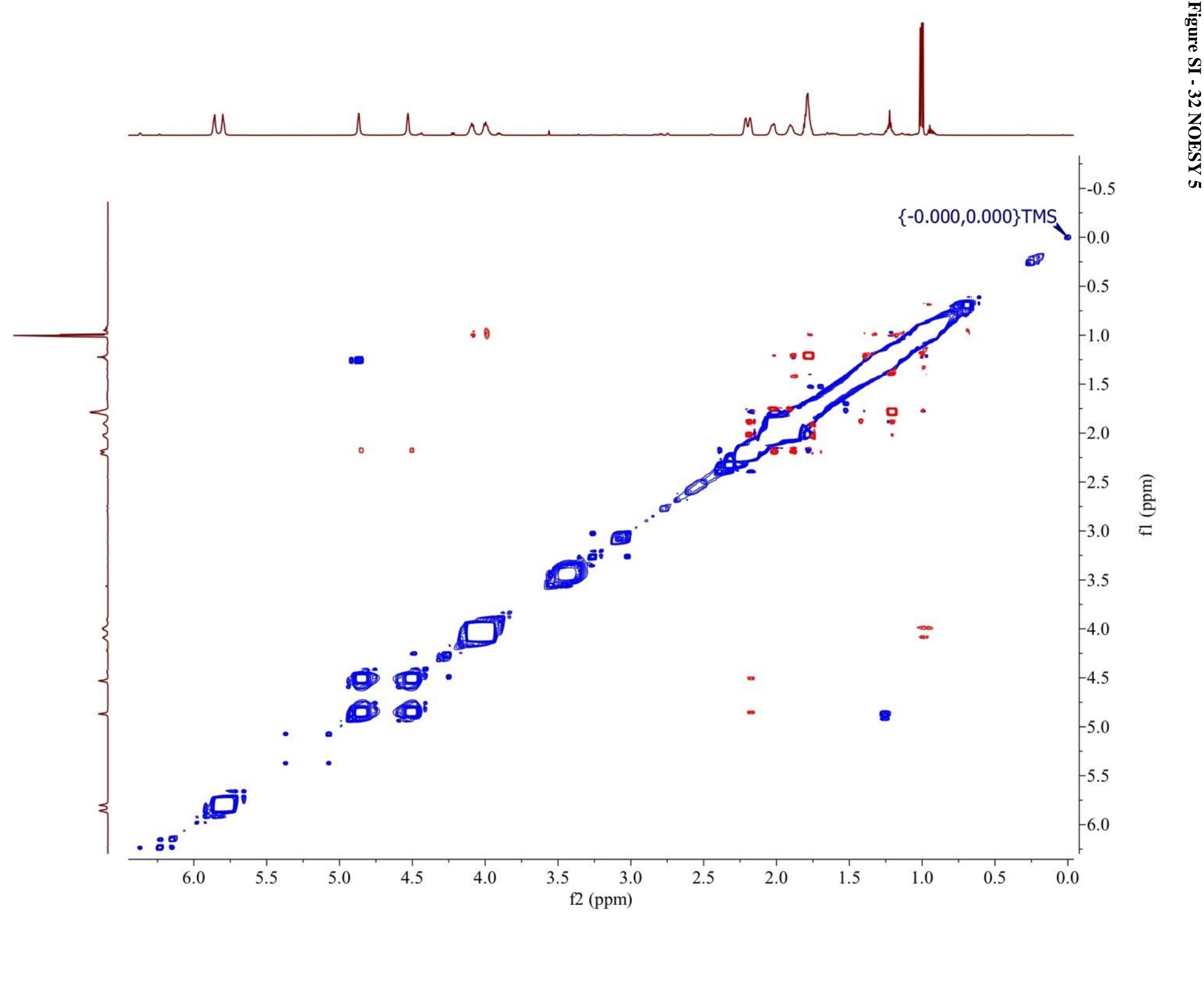




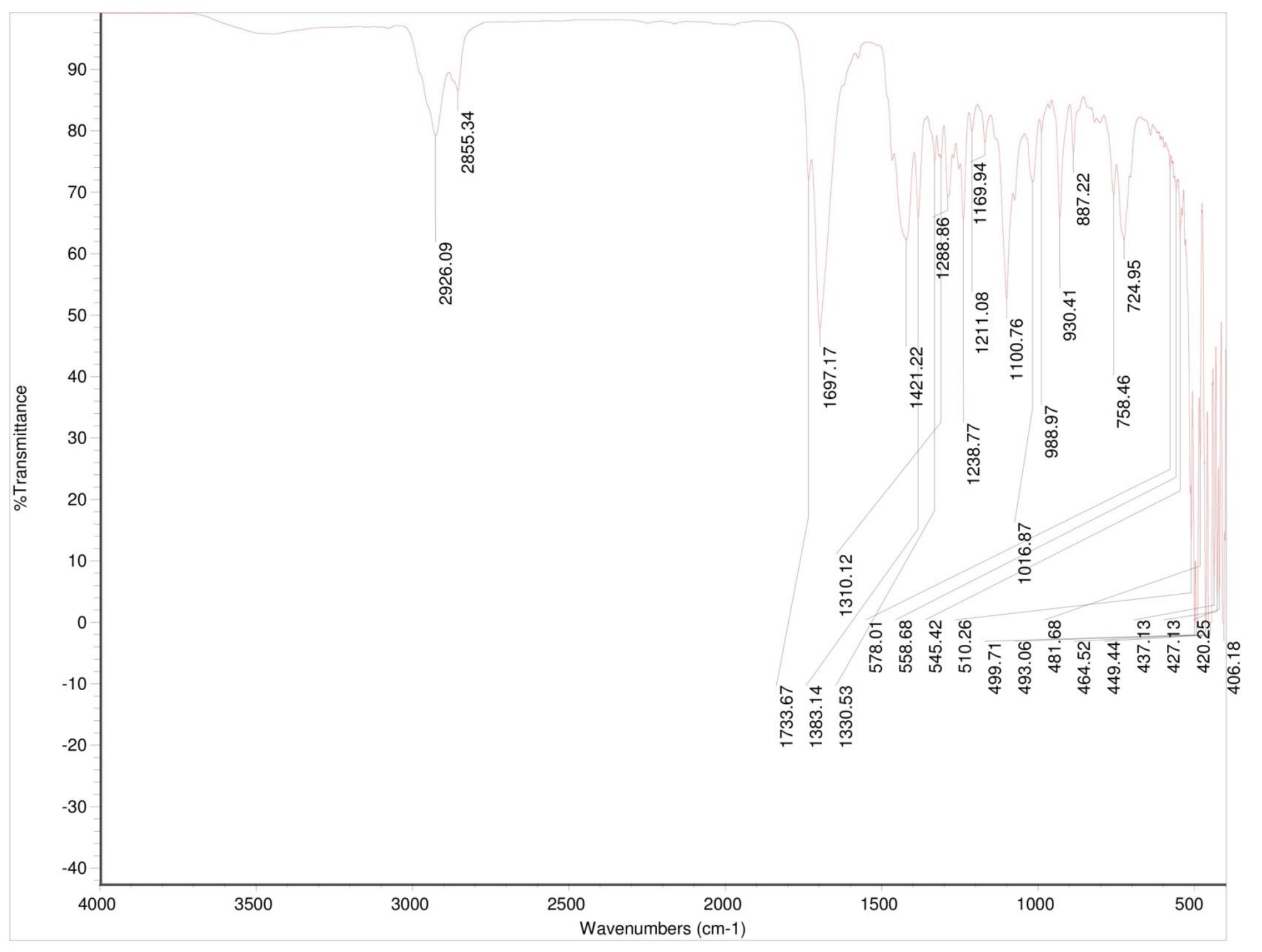


RT: 0.00 - 14.99

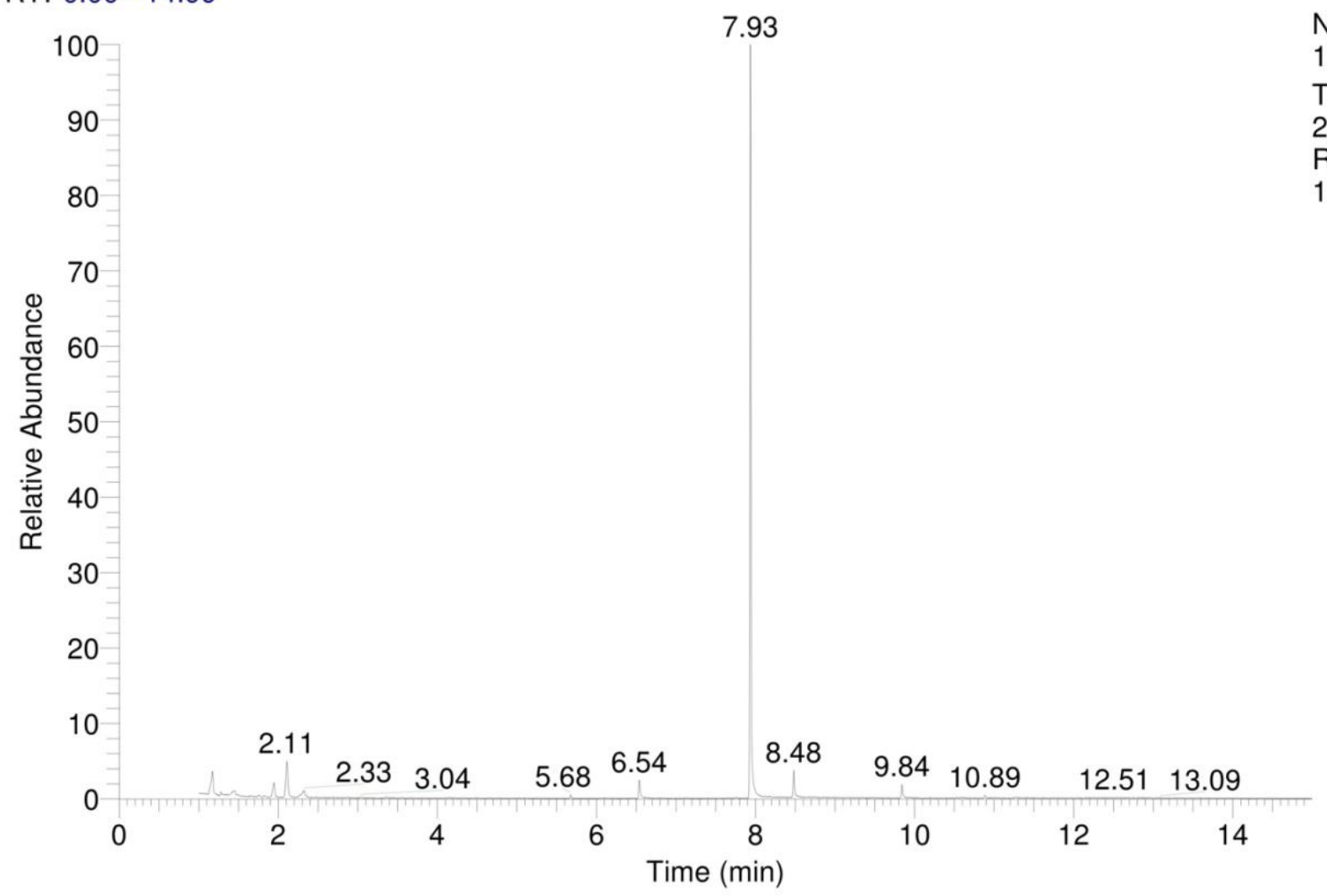

NL:

1.50E8 TIC MS 2018-4-23RLJMPW$1 \mathrm{GC} 01$

2018-4-23-RLJMPW-1GC01 \#1989-2106 RT: 7.76-8.16 AV: 118 NL: 6.79E5

$\mathrm{T}:\{0,0\}+\mathrm{c}$ El Full ms $[90.00-500.00]$

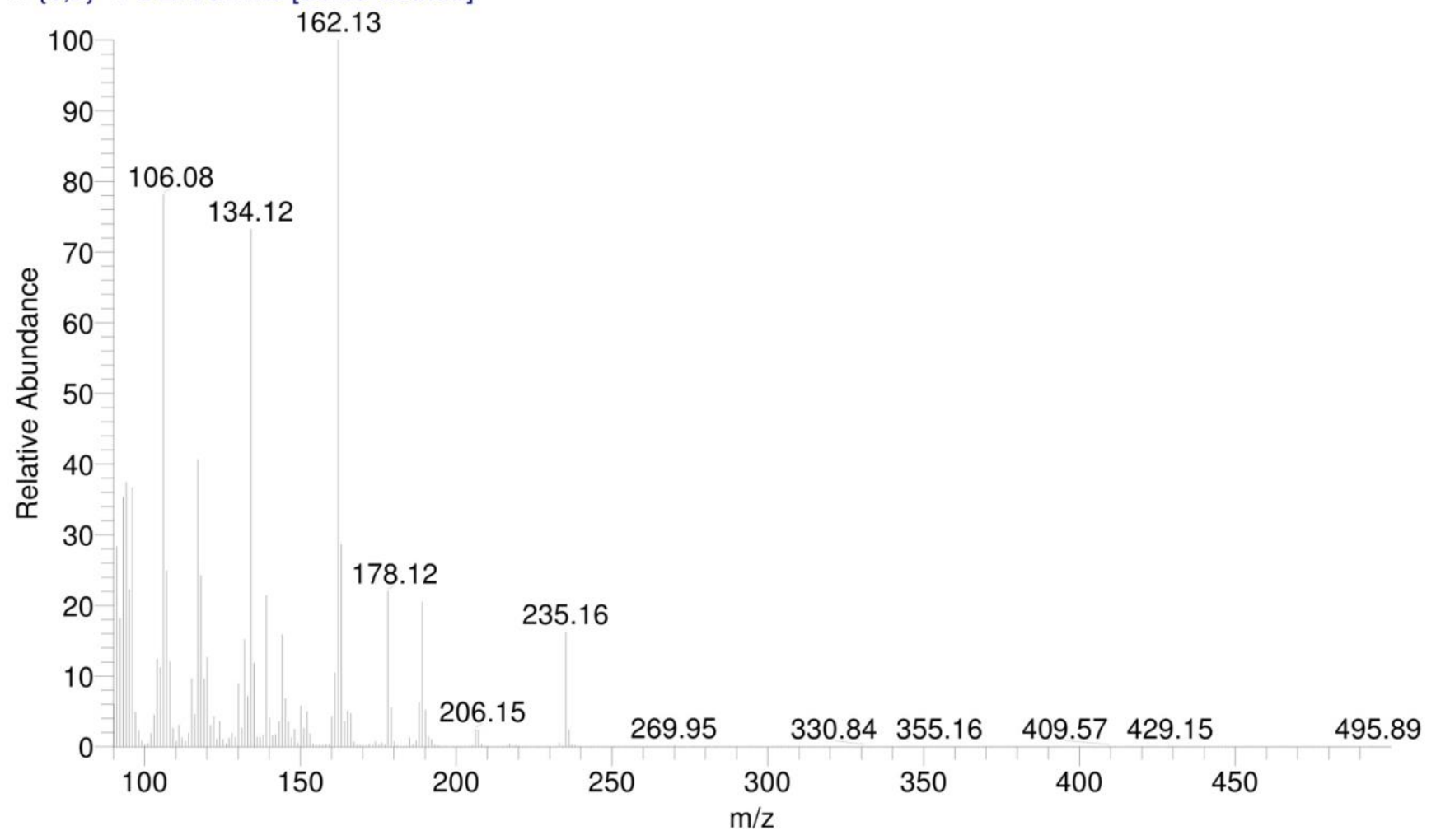


Figure SI - 35 High Resolution Mass Spectroscopy 5

Target Compound Screening Report

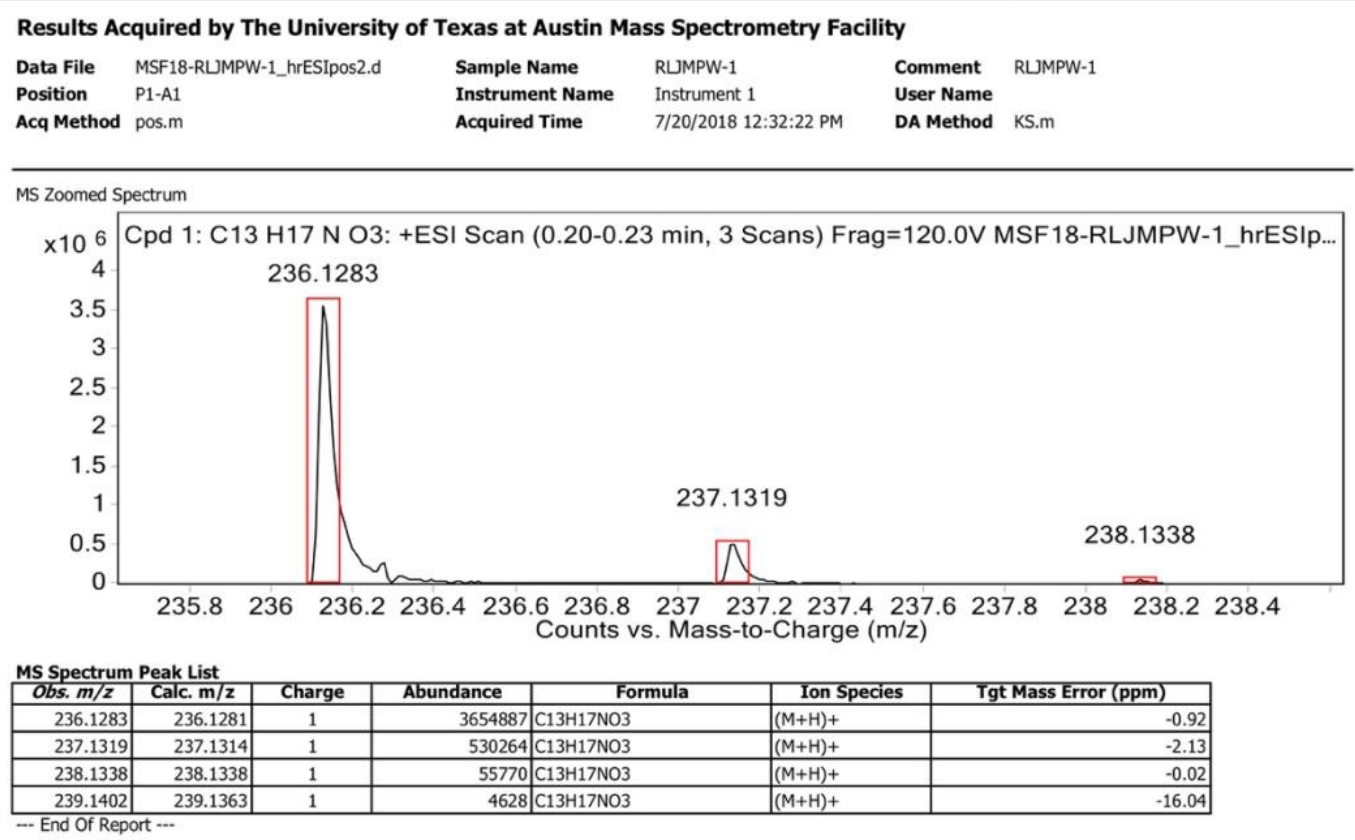


${ }^{1} \mathrm{H}$ NMR $(850 \mathrm{MHz}$, Chloroform- $d) \delta 7.69(\mathrm{~d}, J=8.4 \mathrm{~Hz}, 2 \mathrm{H}), 7.52(\mathrm{t}, J=7.4 \mathrm{~Hz}, 1 \mathrm{H}), 7.43(\mathrm{t}, J$ $=7.8 \mathrm{~Hz}, 2 \mathrm{H}), 5.75(\mathrm{~s}, 2 \mathrm{H}), 4.67(\mathrm{~s}, 2 \mathrm{H}), 2.49-2.46(\mathrm{~m}, 2 \mathrm{H}), 2.40(\mathrm{dt}, J=14.0,7.1 \mathrm{~Hz}, 2 \mathrm{H}), 2.32$ $(\mathrm{dp}, J=13.5,6.7 \mathrm{~Hz}, 1 \mathrm{H}), 2.09(\mathrm{dt}, J=14.2,7.2 \mathrm{~Hz}, 2 \mathrm{H}), 1.46(\mathrm{dp}, J=14.8,7.4 \mathrm{~Hz}, 1 \mathrm{H})$

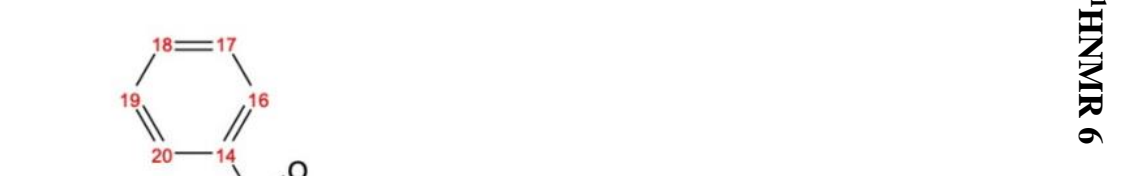


${ }^{13} \mathrm{CNMR}\left(214 \mathrm{MHz}, \mathrm{CDCl}_{3}\right) \delta 214.193,138.577,134.233,132.979,129.179,127.618,65.727$, $51.352,29.426,19.754$.

ฮั

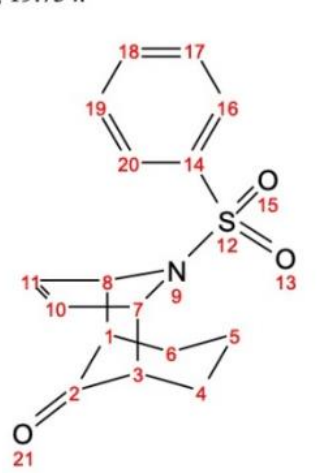

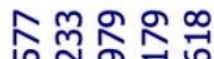

น

岗色

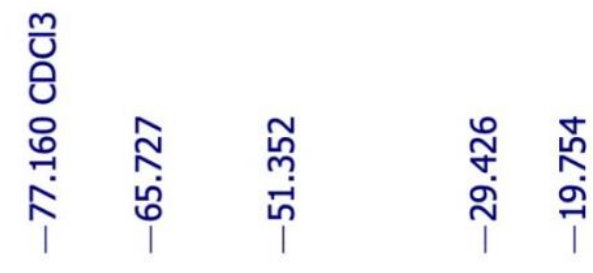

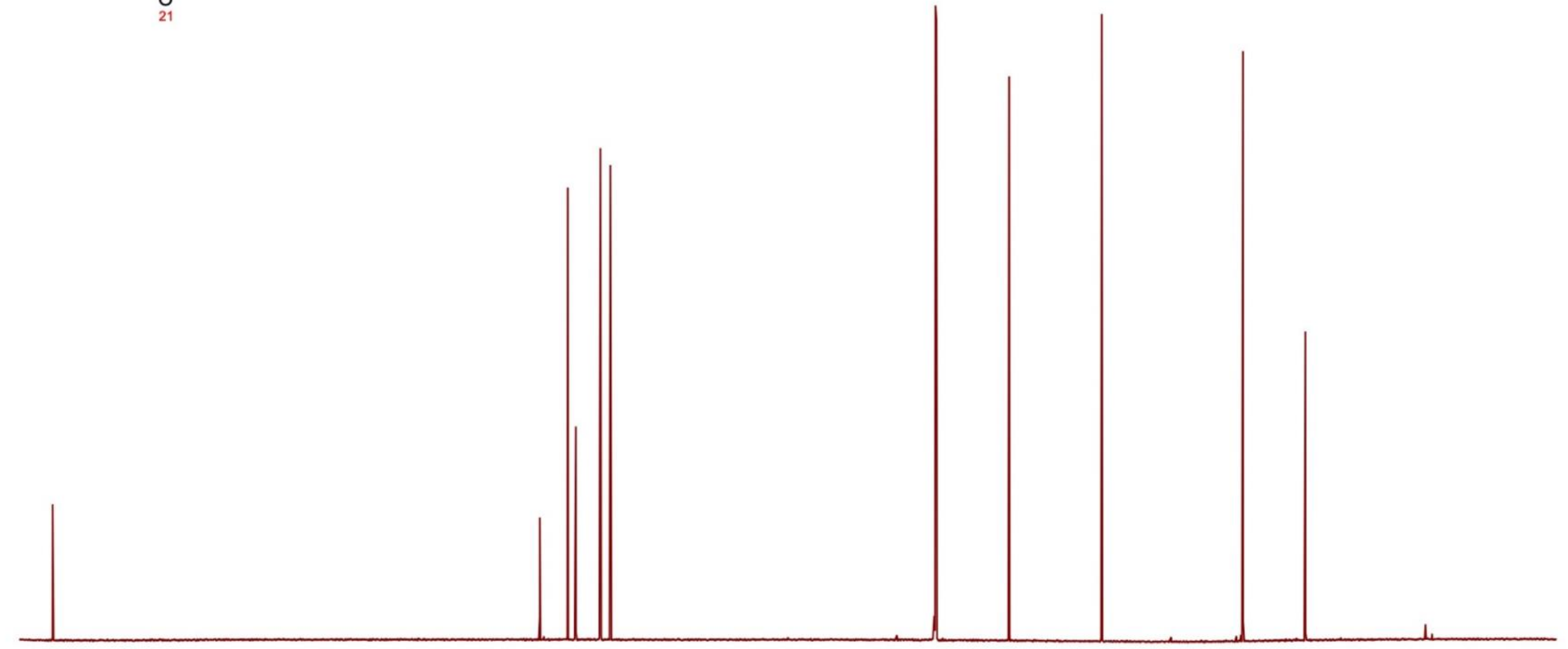

$\begin{array}{lllllll}210 & 200 & 190 & 180 & 170 & 160 & 150\end{array}$

140

120

$\begin{array}{ll}100 & 100 \\ \text { fl }(\mathrm{ppm})\end{array}$ 


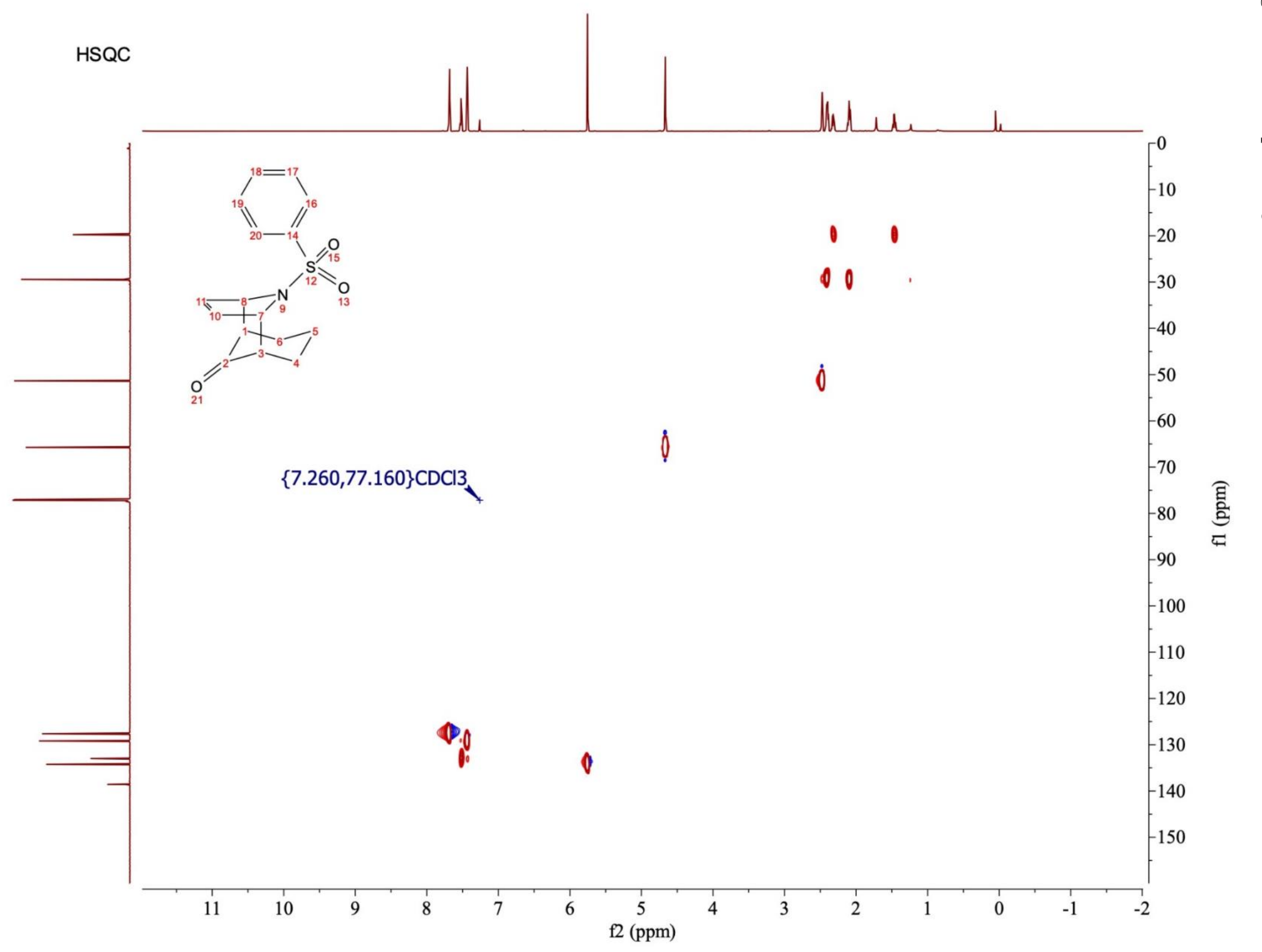




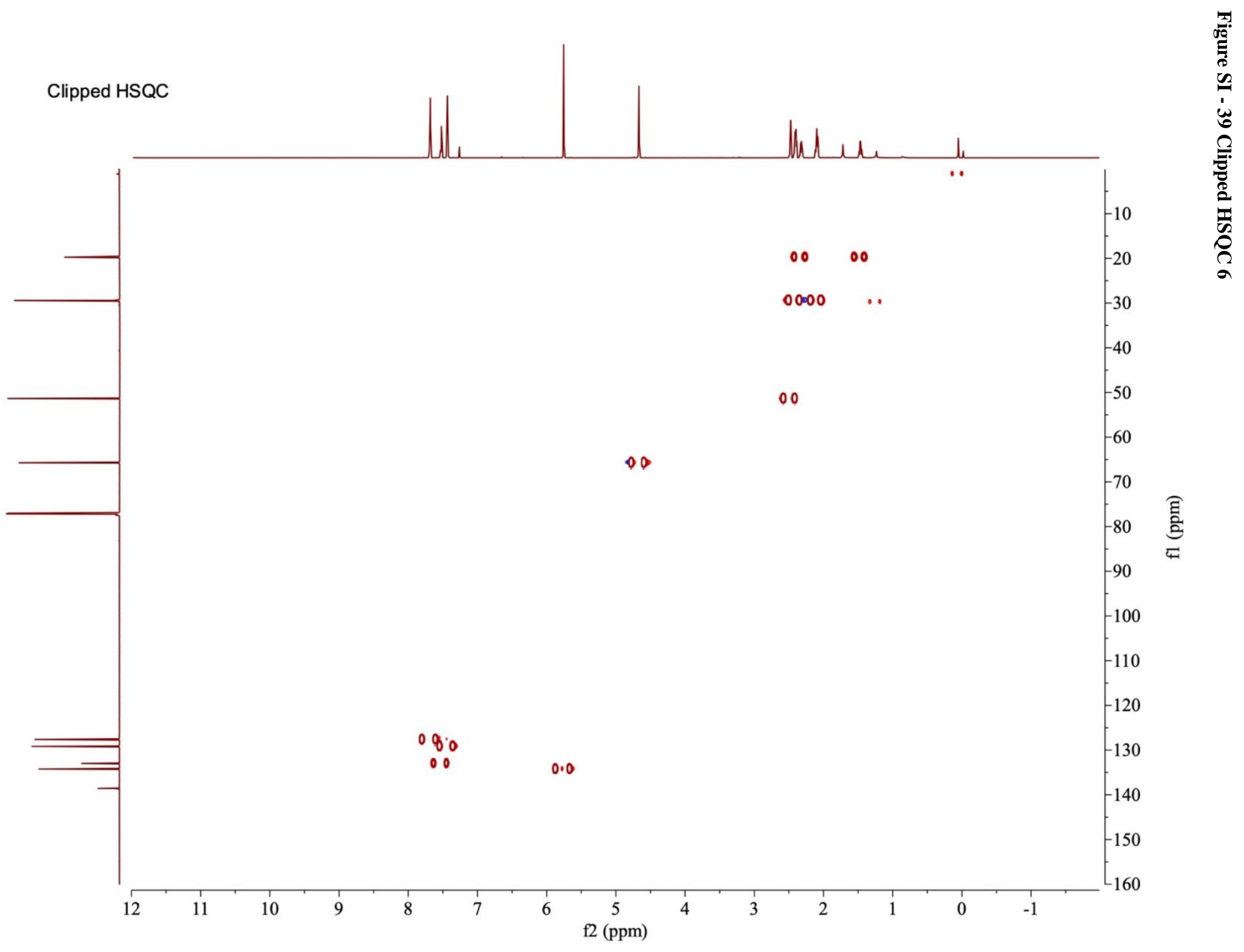




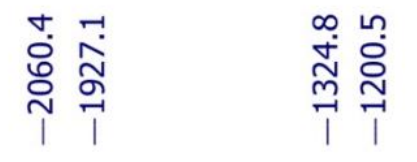

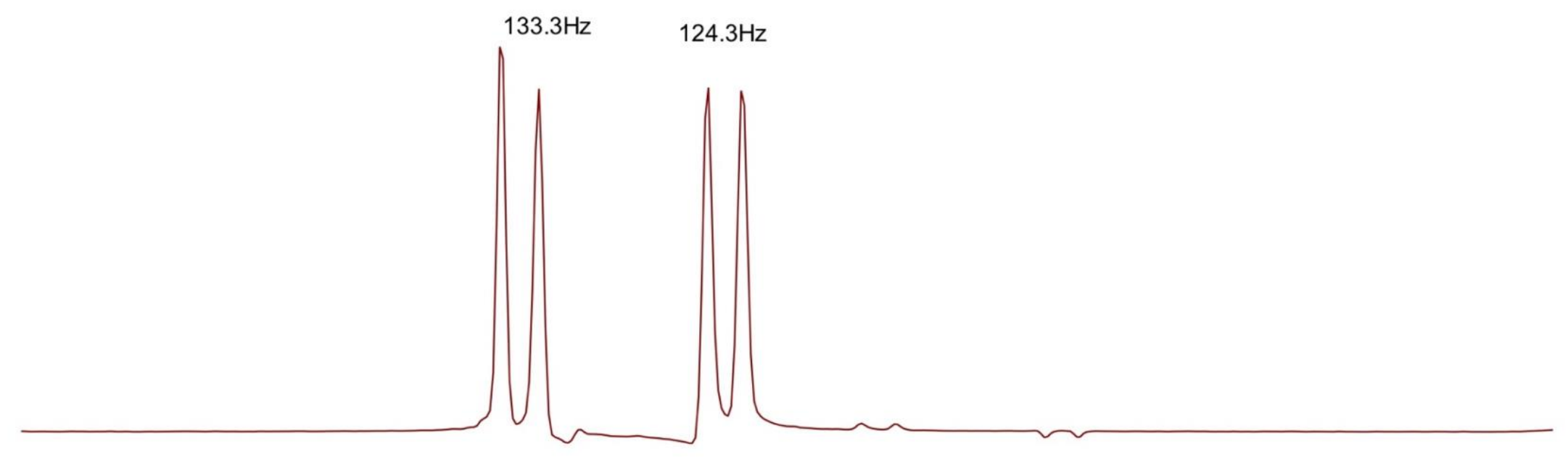

$\begin{array}{lllllllllllllllllllllllllllllllllllllllllllll}1.4 & 4.2 & 4.0 & 3.8 & 3.6 & 3.4 & 3.2 & 3.0 & 2.8 & 2.6 & 2.4 & 2.2 & 2.0 & 1.8 & 1.6 & 1.4 & 1.2 & 1.0 & 0.8 & 0.6 & 0.4 & 0.2 & 0.0 & -0.2 & -0.4 & -0.6 & -0.8 & -1.0 & -1.2 & -1.4 & -1.6 & -1.8\end{array}$ fl (ppm) 


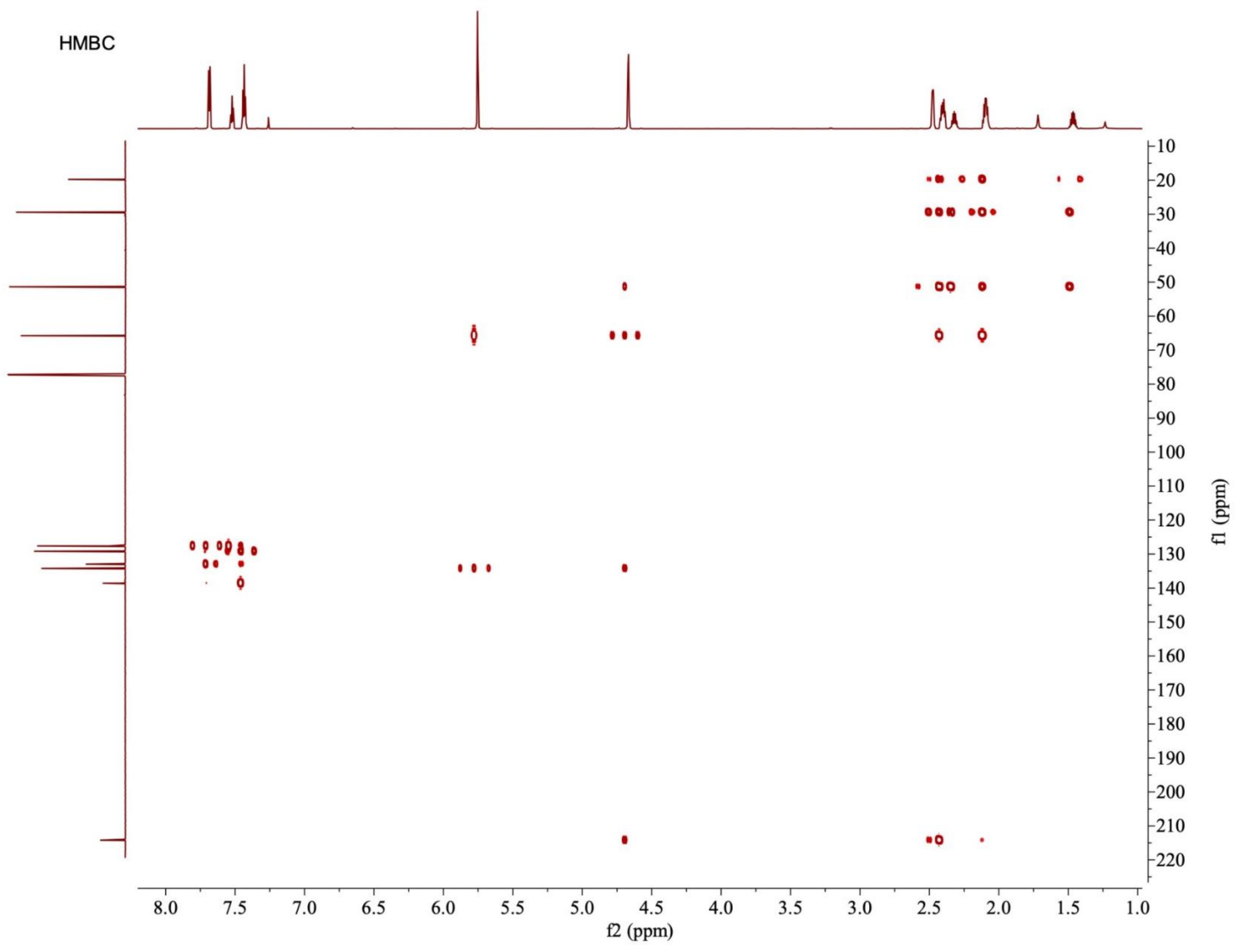




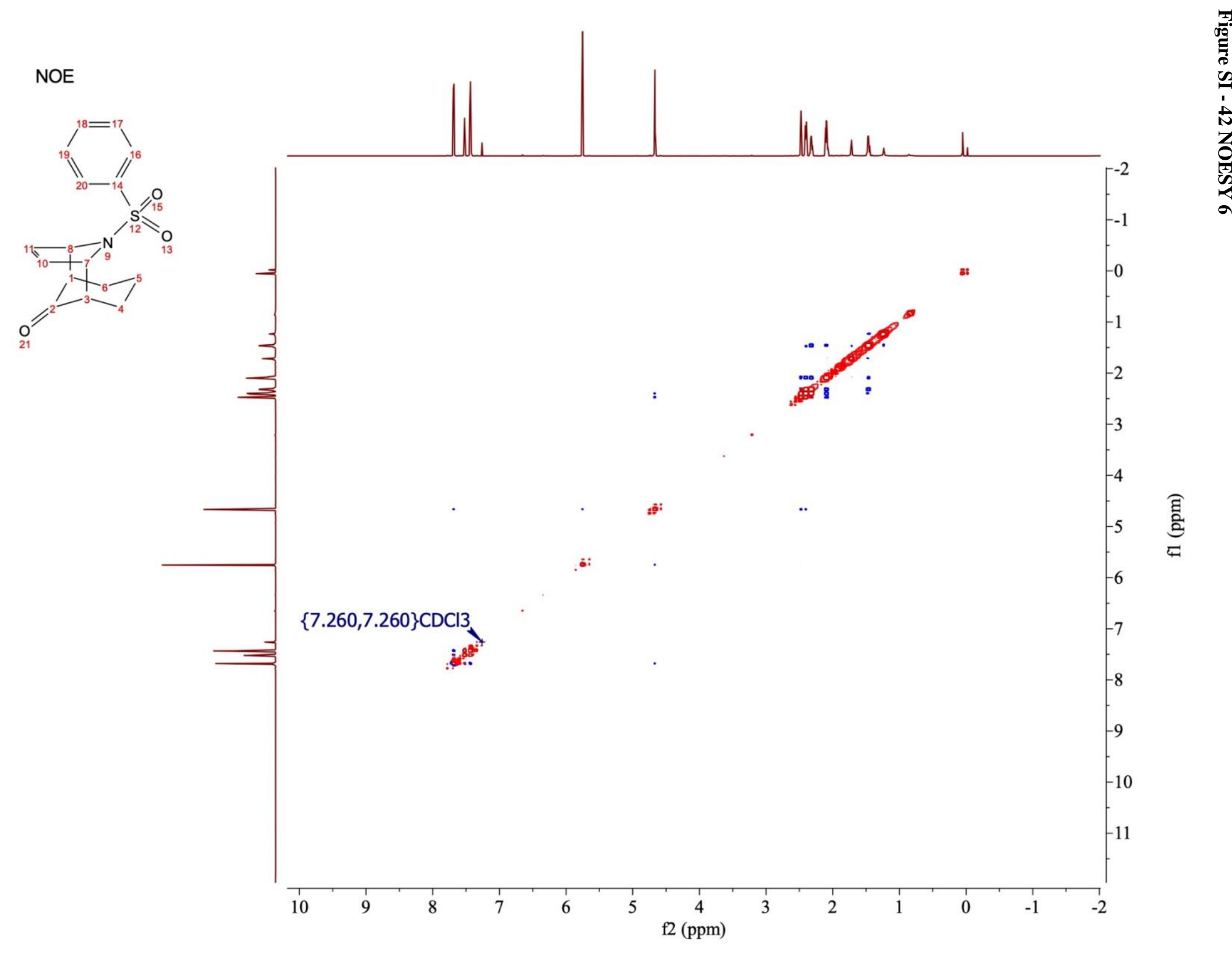




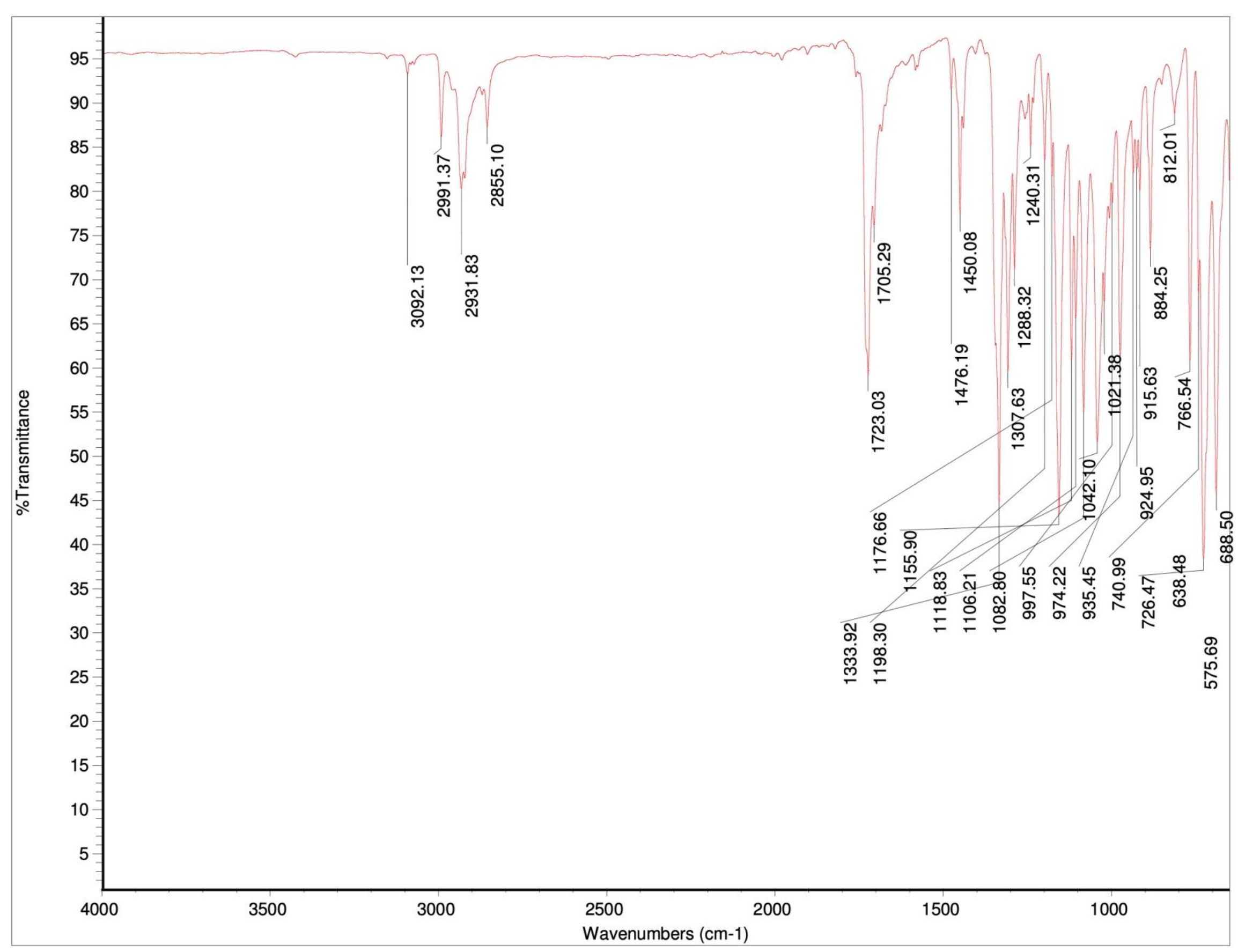


Figure SI - 44 High Resolution Mass Spectroscopy 6

Target Compound Screening Report

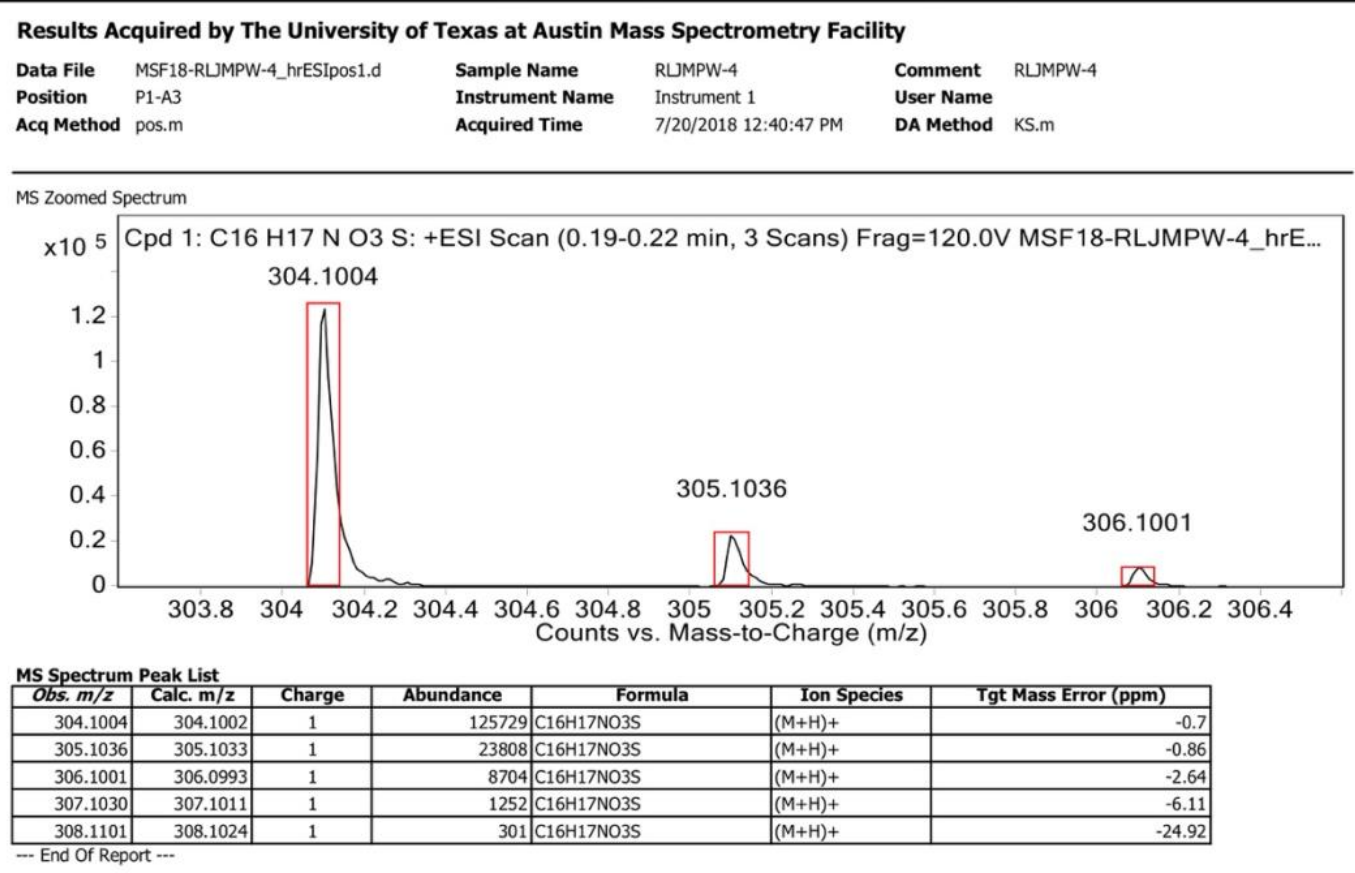


Table SI - 7 Crystallographic Data 6

\begin{tabular}{|c|c|}
\hline Empirical formula & $\mathrm{C}_{16} \mathrm{H}_{17} \mathrm{NO}_{3} \mathrm{~S}$ \\
\hline Formula weight & $303.38 \mathrm{~g} / \mathrm{mol}$ \\
\hline Temperature & $173 \mathrm{~K}$ \\
\hline Wavelength & $1.54184 \AA$ \\
\hline Crystal system & monoclinic \\
\hline Space group & $\mathrm{P} 2{ }_{1} / \mathrm{c}$ \\
\hline Unit cell dimensions & $\begin{array}{l}\mathrm{a}=15.8190(5) \AA, \alpha=90^{\circ} \\
\mathrm{b}=10.2218(3) \AA, \beta=103.292^{\circ} \\
\mathrm{c}=13.7597(5) \AA, \gamma=90^{\circ}\end{array}$ \\
\hline Volume & $1400.75 \AA^{3}$ \\
\hline $\mathbf{Z}$ & 4 \\
\hline Density (calculated) & $1.242 \mathrm{~g} / \mathrm{cm}^{3}$ \\
\hline Absorption coefficient & $0.661 \mathrm{~mm}^{-1}$ \\
\hline $\mathbf{F}(\mathbf{0 0 0})$ & 640 \\
\hline$\theta_{\max }$ & $70.85^{\circ}$ \\
\hline Crystal size $\left(\mathrm{mm}^{\mathbf{3}}\right)$ & $0.161 \times 0.218 \times 0.304 \mathrm{~mm}$ \\
\hline Exposure time & $17.42 \mathrm{hr}$ \\
\hline $\begin{array}{l}\text { Reflections } \\
\text { collected }\end{array}$ & $\begin{array}{l}9113 \\
{\left[\mathrm{R}_{\text {int }}=3.77 \%\right]}\end{array}$ \\
\hline Independent reflections & 2615 \\
\hline Completeness to $\theta \max$ & $98.7 \%$ \\
\hline Min./max. transmission & 0.738 \\
\hline Refinement method & Full matrix least squares on F2 \\
\hline Absorption correction & Numerical \\
\hline Goodness-of-fit & 1.093 \\
\hline $\begin{array}{l}\text { Final } R \text { indices } \\
{[I>\mathbf{I \theta}(\mathrm{I})]}\end{array}$ & $5.57 \%$ \\
\hline $\mathbf{R}$ indices (all) & $14.53 \%$ \\
\hline $\begin{array}{l}\text { Largest diff. peak } \\
\text { and hole }\end{array}$ & $0.710,-0.375 \mathrm{e}^{-} / \mathrm{A}^{3}$ \\
\hline
\end{tabular}

Figure SI - 45 S3 X-Ray crystal structure. Atomic displacement ellipsoids generated at $50 \%$ probability

Recrystallized from a minimum of hot hexanes.
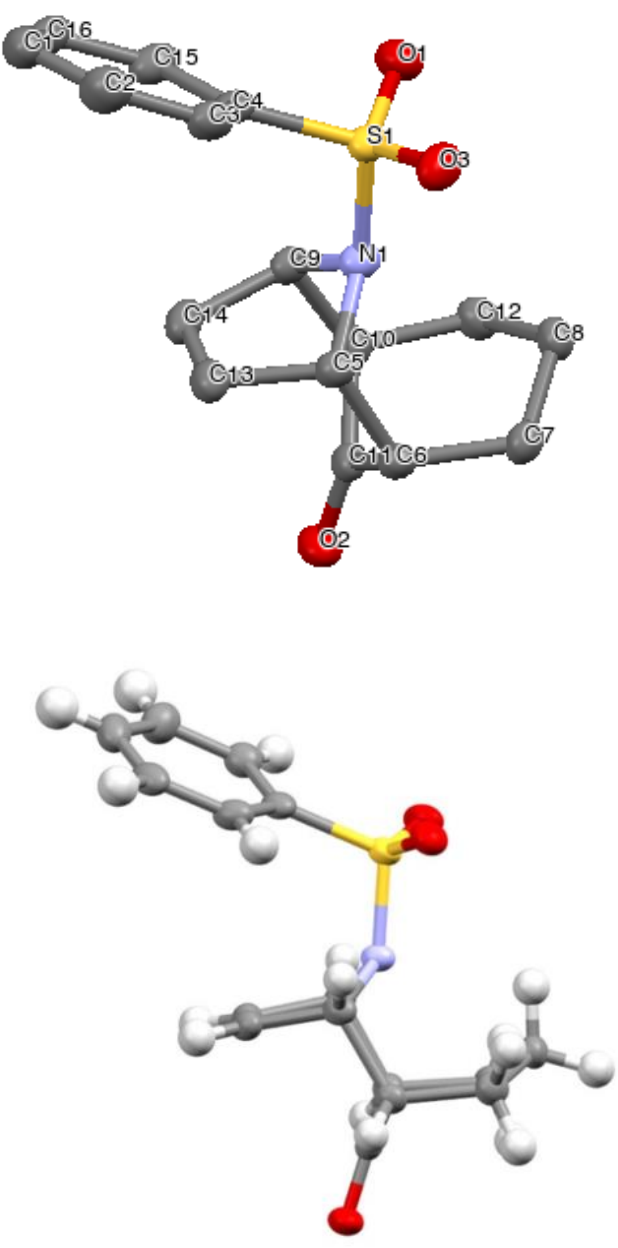
Table SI - 8 Dihedral Angles $\left({ }^{\circ}\right) 6$

\begin{tabular}{|c|c|c|c|c|}
\hline Number & Atom1 & Atom2 & Atom3 & Angle $\left(^{\circ}\right)$ \\
\hline 1 & $\mathrm{O} 1$ & S1 & $\mathrm{O} 3$ & $120.0(1)$ \\
\hline 2 & O1 & S1 & N1 & $105.0(1)$ \\
\hline 3 & $\mathrm{O} 1$ & S1 & $\mathrm{C} 4$ & $107.7(1)$ \\
\hline 4 & $\mathrm{O} 3$ & S1 & N1 & 105.6(1) \\
\hline 5 & $\mathrm{O} 3$ & S1 & C4 & $108.3(1)$ \\
\hline 6 & N1 & S1 & $\mathrm{C} 4$ & 110.1(1) \\
\hline 7 & S1 & N1 & C5 & $122.0(1)$ \\
\hline 8 & S1 & N1 & C9 & 119.1(1) \\
\hline 9 & C5 & N1 & C9 & $102.6(2)$ \\
\hline 10 & H1 & $\mathrm{C} 1$ & $\mathrm{C} 2$ & 120.0 \\
\hline 11 & H1 & $\mathrm{C} 1$ & C16 & 120.0 \\
\hline 12 & $\mathrm{C} 2$ & $\mathrm{C} 1$ & C16 & $120.0(2)$ \\
\hline 13 & $\mathrm{C} 1$ & $\mathrm{C} 2$ & H17 & 119.8 \\
\hline 14 & $\mathrm{C} 1$ & $\mathrm{C} 2$ & C3 & $120.4(2)$ \\
\hline 15 & H17 & $\mathrm{C} 2$ & $\mathrm{C} 3$ & 119.8 \\
\hline 16 & $\mathrm{C} 2$ & C3 & H16 & 120.5 \\
\hline 17 & C2 & C3 & C4 & $118.9(2)$ \\
\hline 18 & H16 & C3 & C4 & 120.6 \\
\hline 19 & S1 & $\mathrm{C} 4$ & C3 & $119.8(2)$ \\
\hline 20 & S1 & $\mathrm{C} 4$ & C15 & $118.9(2)$ \\
\hline 21 & C3 & $\mathrm{C} 4$ & C15 & $121.4(2)$ \\
\hline 22 & N1 & C5 & H8 & 113.2 \\
\hline 23 & N1 & C5 & C6 & $106.6(2)$ \\
\hline 24 & N1 & C5 & $\mathrm{C} 13$ & $101.7(2)$ \\
\hline 25 & $\mathrm{H} 8$ & C5 & C6 & 113.2 \\
\hline 26 & $\mathrm{H} 8$ & C5 & $\mathrm{C} 13$ & 113.2 \\
\hline 27 & C6 & C5 & $\mathrm{C} 13$ & $108.1(2)$ \\
\hline 28 & C5 & C6 & H11 & 109.3 \\
\hline 29 & C5 & C6 & C7 & $115.4(2)$ \\
\hline 30 & C5 & C6 & C11 & $108.1(2)$ \\
\hline 31 & H11 & C6 & $\mathrm{C} 7$ & 109.3 \\
\hline 32 & H11 & C6 & C11 & 109.3 \\
\hline 33 & C7 & C6 & C11 & $105.2(2)$ \\
\hline 34 & C6 & C7 & $\mathrm{H} 7$ & 108.6 \\
\hline 35 & C6 & C7 & H6 & 108.6 \\
\hline 36 & C6 & C7 & C8 & $114.7(2)$ \\
\hline 37 & $\mathrm{H} 7$ & C7 & H6 & 107.6 \\
\hline 38 & $\mathrm{H} 7$ & C7 & C8 & 108.6 \\
\hline 39 & H6 & C7 & C8 & 108.6 \\
\hline
\end{tabular}




\begin{tabular}{|c|c|c|c|c|}
\hline 40 & C7 & $\mathrm{C} 8$ & H5 & 108.6 \\
\hline 41 & $\mathrm{C} 7$ & $\mathrm{C} 8$ & $\mathrm{H} 2$ & 108.7 \\
\hline 42 & C7 & C8 & $\mathrm{C} 12$ & $114.5(2)$ \\
\hline 43 & H5 & $\mathrm{C} 8$ & $\mathrm{H} 2$ & 107.5 \\
\hline 44 & H5 & $\mathrm{C} 8$ & $\mathrm{C} 12$ & 108.7 \\
\hline 45 & $\mathrm{H} 2$ & C8 & C12 & 108.6 \\
\hline 46 & N1 & C9 & H13 & 113.1 \\
\hline 47 & N1 & C9 & $\mathrm{C} 10$ & $105.6(2)$ \\
\hline 48 & N1 & C9 & C14 & $101.6(2)$ \\
\hline 49 & H13 & C9 & C10 & 113.1 \\
\hline 50 & H13 & C9 & C14 & 113.0 \\
\hline 51 & $\mathrm{C} 10$ & C9 & C14 & $109.7(2)$ \\
\hline 52 & C9 & $\mathrm{C} 10$ & H12 & 109.6 \\
\hline 53 & C9 & $\mathrm{C} 10$ & C11 & $107.2(2)$ \\
\hline 54 & C9 & $\mathrm{C} 10$ & $\mathrm{C} 12$ & $114.7(2)$ \\
\hline 55 & H12 & $\mathrm{C} 10$ & $\mathrm{C} 11$ & 109.6 \\
\hline 56 & H12 & $\mathrm{C} 10$ & C12 & 109.7 \\
\hline 57 & C11 & $\mathrm{C} 10$ & C12 & $105.8(2)$ \\
\hline 58 & $\mathrm{O} 2$ & C11 & C6 & 124.1(2) \\
\hline 59 & $\mathrm{O} 2$ & C11 & C10 & $124.3(2)$ \\
\hline 60 & C6 & C11 & C10 & $111.4(2)$ \\
\hline 61 & C8 & $\mathrm{C} 12$ & C10 & $115.6(2)$ \\
\hline 62 & C8 & $\mathrm{C} 12$ & $\mathrm{H} 4$ & 108.4 \\
\hline 63 & C8 & $\mathrm{C} 12$ & $\mathrm{H} 3$ & 108.4 \\
\hline 64 & C10 & $\mathrm{C} 12$ & $\mathrm{H} 4$ & 108.4 \\
\hline 65 & C10 & $\mathrm{C} 12$ & $\mathrm{H} 3$ & 108.4 \\
\hline 66 & $\mathrm{H} 4$ & $\mathrm{C} 12$ & $\mathrm{H} 3$ & 107.4 \\
\hline 67 & $\mathrm{C} 5$ & $\mathrm{C} 13$ & H10 & 125.7 \\
\hline 68 & $\mathrm{C} 5$ & $\mathrm{C} 13$ & C14 & $108.7(2)$ \\
\hline 69 & H10 & $\mathrm{C} 13$ & C14 & 125.7 \\
\hline 70 & C9 & C14 & C13 & $108.9(2)$ \\
\hline 71 & C9 & $\mathrm{C} 14$ & H9 & 125.5 \\
\hline 72 & $\mathrm{C} 13$ & C14 & H9 & 125.6 \\
\hline 73 & $\mathrm{C} 4$ & $\mathrm{C} 15$ & H15 & 120.5 \\
\hline 74 & C4 & $\mathrm{C} 15$ & C16 & $119.0(2)$ \\
\hline 75 & H15 & $\mathrm{C} 15$ & C16 & 120.5 \\
\hline 76 & $\mathrm{C} 1$ & C16 & C15 & $120.4(2)$ \\
\hline 77 & $\mathrm{C} 1$ & C16 & H14 & 119.8 \\
\hline 78 & C15 & C16 & H14 & 119.8 \\
\hline
\end{tabular}


Table SI - 9 Bond Length (Å) 6

\begin{tabular}{|c|c|c|c|}
\hline Number & Atom1 & Atom2 & Length \\
\hline 1 & $\mathrm{~S} 1$ & $\mathrm{O} 1$ & $1.430(2)$ \\
\hline 2 & $\mathrm{~S} 1$ & $\mathrm{O} 3$ & $1.432(2)$ \\
\hline 3 & $\mathrm{~S} 1$ & N1 & $1.638(2)$ \\
\hline 4 & $\mathrm{~S} 1$ & $\mathrm{C} 4$ & $1.767(3)$ \\
\hline 5 & $\mathrm{O} 2$ & $\mathrm{C} 11$ & $1.212(3)$ \\
\hline 6 & N1 & $\mathrm{C} 5$ & $1.476(2)$ \\
\hline 7 & N1 & C9 & $1.477(3)$ \\
\hline 8 & $\mathrm{C} 1$ & $\mathrm{H} 1$ & 0.950 \\
\hline 9 & $\mathrm{C} 1$ & $\mathrm{C} 2$ & $1.392(4)$ \\
\hline 10 & $\mathrm{C} 1$ & $\mathrm{C} 16$ & $1.381(4)$ \\
\hline 11 & $\mathrm{C} 2$ & H17 & 0.950 \\
\hline 12 & $\mathrm{C} 2$ & $\mathrm{C} 3$ & $1.386(4)$ \\
\hline 13 & $\mathrm{C} 3$ & H16 & 0.950 \\
\hline 14 & $\mathrm{C} 3$ & $\mathrm{C} 4$ & $1.386(4)$ \\
\hline 15 & $\mathrm{C} 4$ & $\mathrm{C} 15$ & $1.390(4)$ \\
\hline 16 & $\mathrm{C} 5$ & H8 & 1.000 \\
\hline 17 & $\mathrm{C} 5$ & C6 & $1.558(3)$ \\
\hline 18 & $\mathrm{C} 5$ & $\mathrm{C} 13$ & $1.522(3)$ \\
\hline 19 & C6 & H11 & 1.000 \\
\hline 20 & C6 & $\mathrm{C} 7$ & $1.540(3)$ \\
\hline 21 & C6 & $\mathrm{C} 11$ & $1.518(3)$ \\
\hline 22 & $\mathrm{C} 7$ & $\mathrm{H} 7$ & 0.989 \\
\hline 23 & $\mathrm{C} 7$ & H6 & 0.990 \\
\hline 24 & $\mathrm{C} 7$ & $\mathrm{C} 8$ & $1.541(3)$ \\
\hline 25 & $\mathrm{C} 8$ & H5 & 0.990 \\
\hline 26 & $\mathrm{C} 8$ & $\mathrm{H} 2$ & 0.990 \\
\hline 27 & $\mathrm{C} 8$ & $\mathrm{C} 12$ & $1.543(4)$ \\
\hline 28 & C9 & $\mathrm{H} 13$ & 1.000 \\
\hline 29 & C9 & $\mathrm{C} 10$ & $1.557(4)$ \\
\hline 30 & C9 & C14 & $1.524(3)$ \\
\hline 31 & $\mathrm{C} 10$ & $\mathrm{H} 12$ & 1.000 \\
\hline 32 & $\mathrm{C} 10$ & $\mathrm{C} 11$ & $1.514(3)$ \\
\hline 33 & $\mathrm{C} 10$ & $\mathrm{C} 12$ & $1.553(3)$ \\
\hline 34 & $\mathrm{C} 12$ & $\mathrm{H} 4$ & 0.991 \\
\hline 35 & $\mathrm{C} 12$ & H3 & 0.990 \\
\hline 36 & $\mathrm{C} 13$ & $\mathrm{H} 10$ & 0.950 \\
\hline 37 & $\mathrm{C} 13$ & C14 & $1.325(3)$ \\
\hline 38 & $\mathrm{C} 14$ & H9 & 0.950 \\
\hline 39 & $\mathrm{C} 15$ & H15 & 0.950 \\
\hline 40 & $\mathrm{C} 15$ & C16 & $1.387(4)$ \\
\hline 41 & C16 & H14 & 0.950 \\
\hline
\end{tabular}




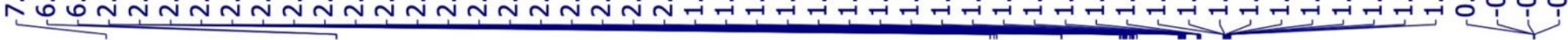

'H NMR $(850 \mathrm{MHz}$, Chloroform- $d$ ) $\delta 6.19-6.04(\mathrm{~m}, 2 \mathrm{H}), 2.78(\mathrm{~s}, 2 \mathrm{H}), 2.75(\mathrm{~d}, J=11.5 \mathrm{~Hz}$,

$1 \mathrm{H}), 2.44-2.39(\mathrm{~m}, 2 \mathrm{H}), 2.13-2.07(\mathrm{~m}, 2 \mathrm{H}), 2.07-2.02(\mathrm{~m}, 2 \mathrm{H}), 1.80(\mathrm{dtt}, J=13.9,7.0,3.5 \mathrm{~Hz}$

$1 \mathrm{H}), 1.74-1.68(\mathrm{~m}, 1 \mathrm{H}), 1.57$ (dddt, $J=14.9,11.0,7.2,3.4 \mathrm{~Hz}, 1 \mathrm{H})$.
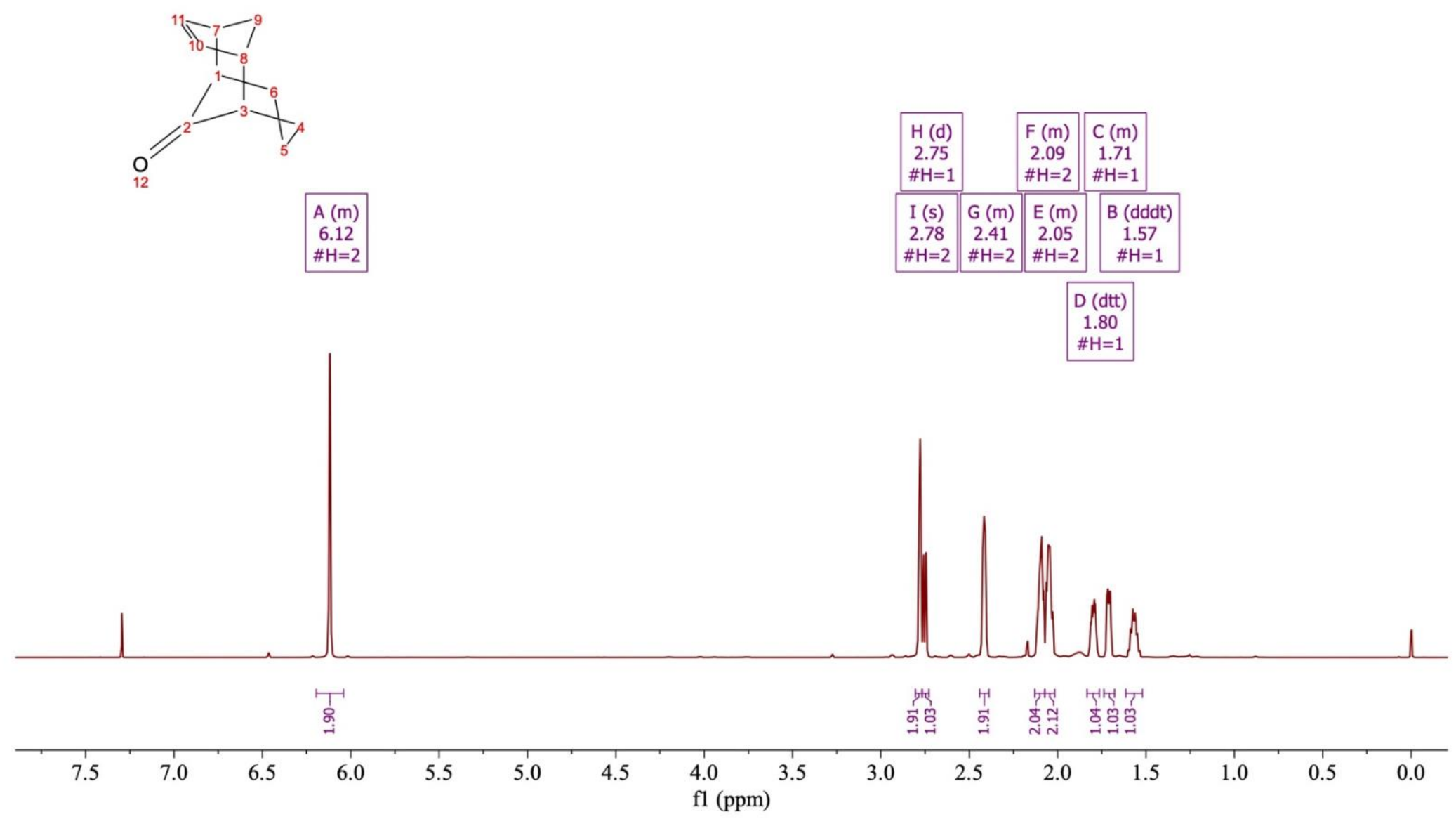

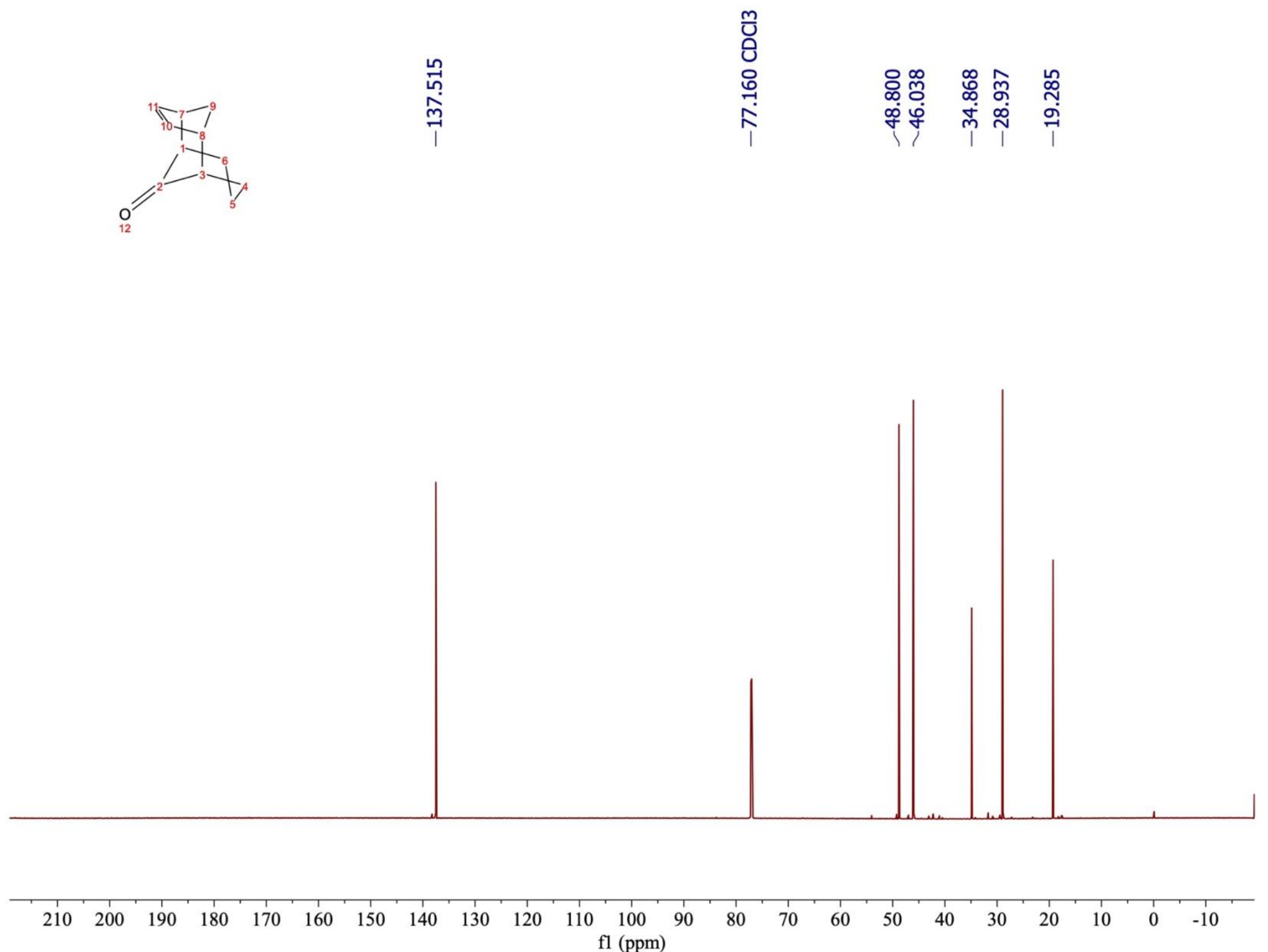


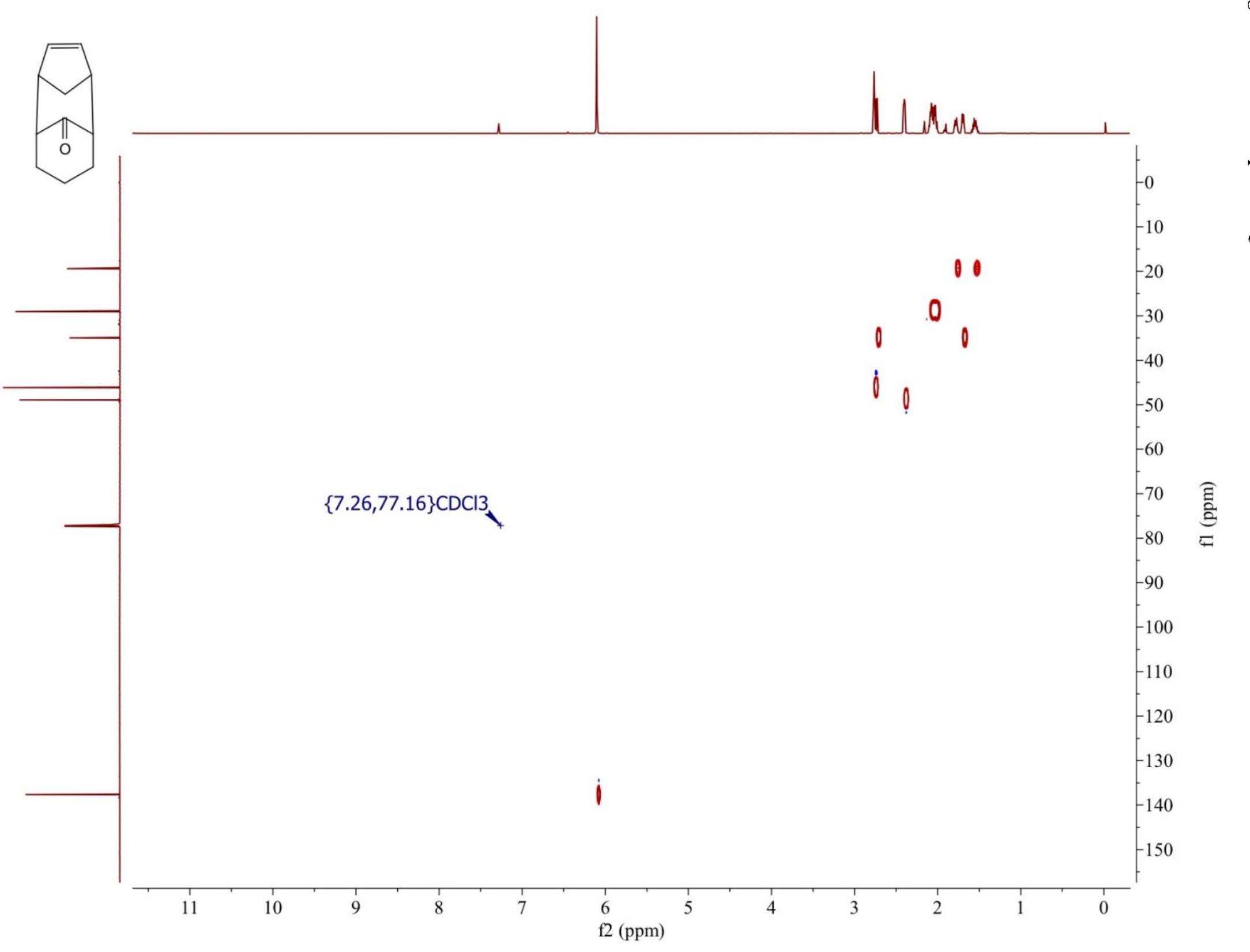

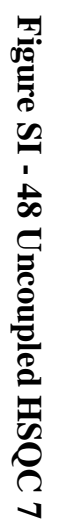




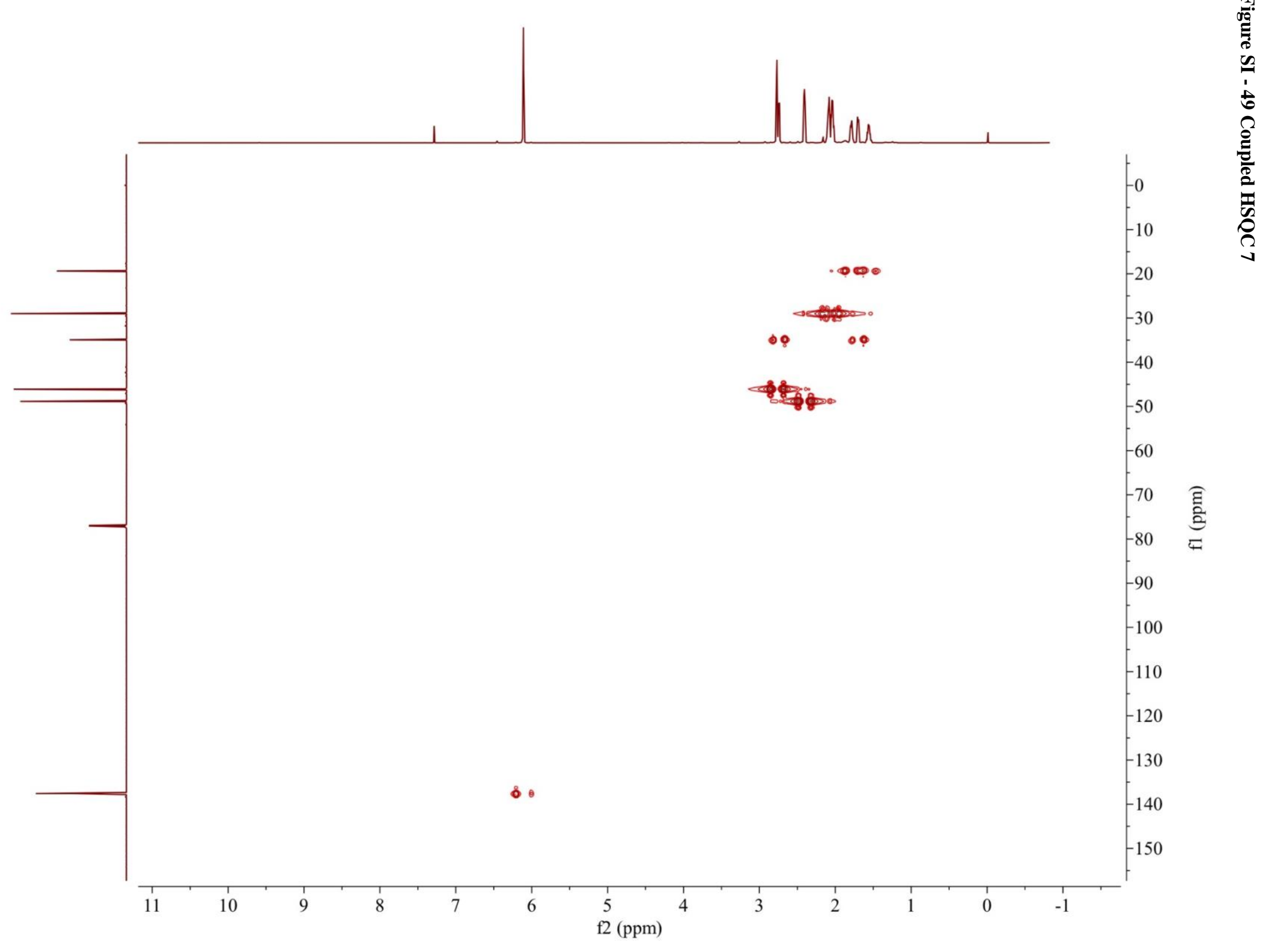




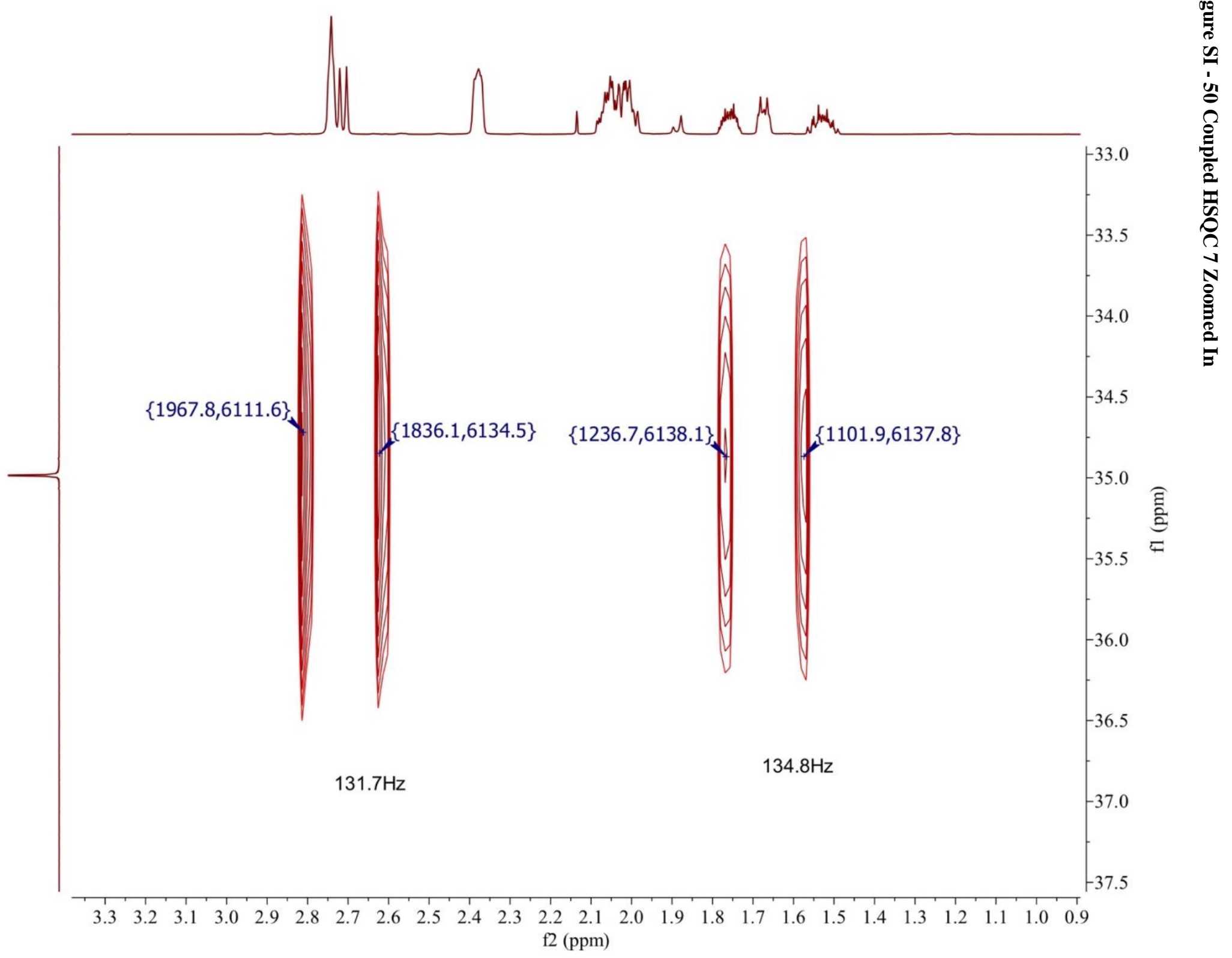

ปิ 


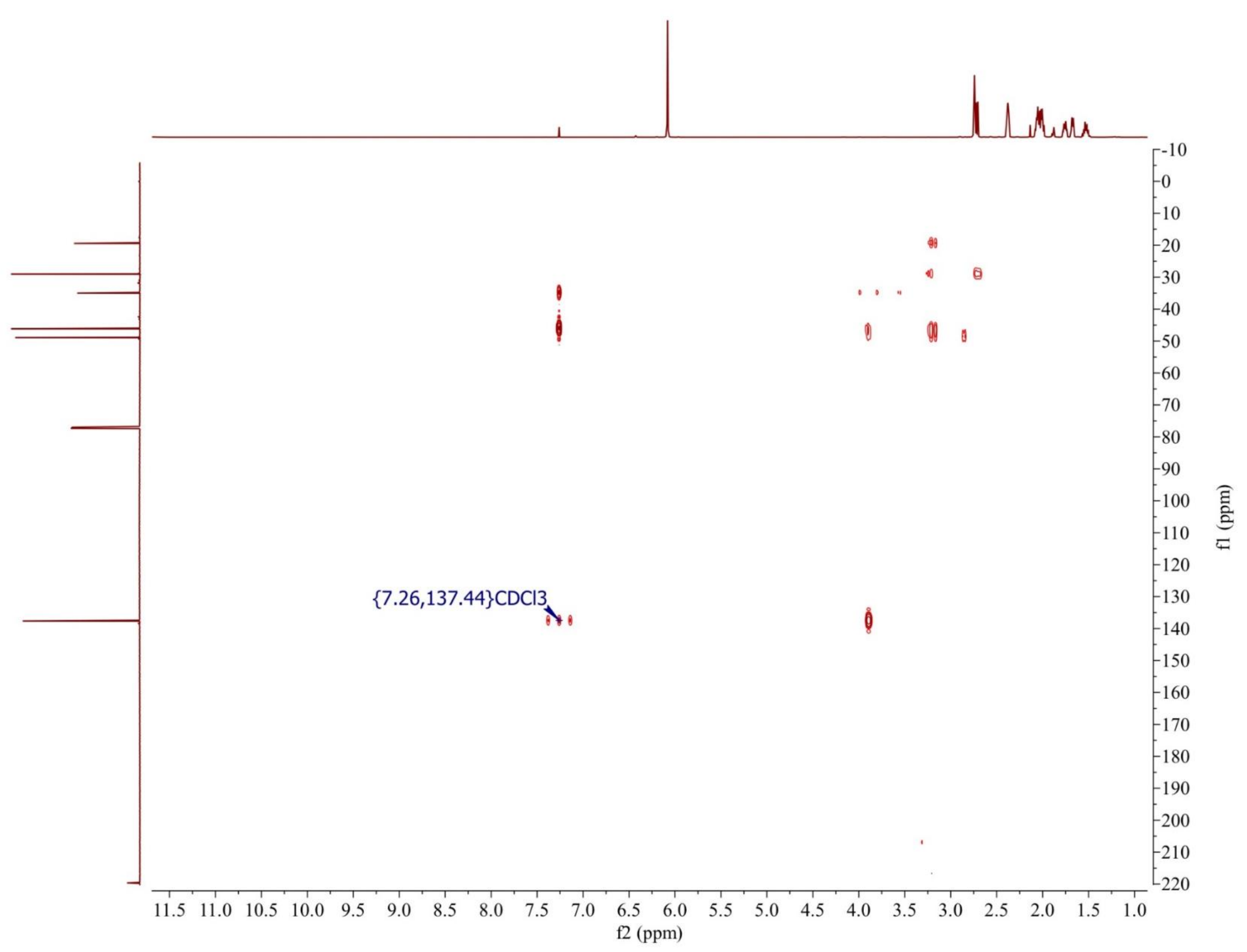




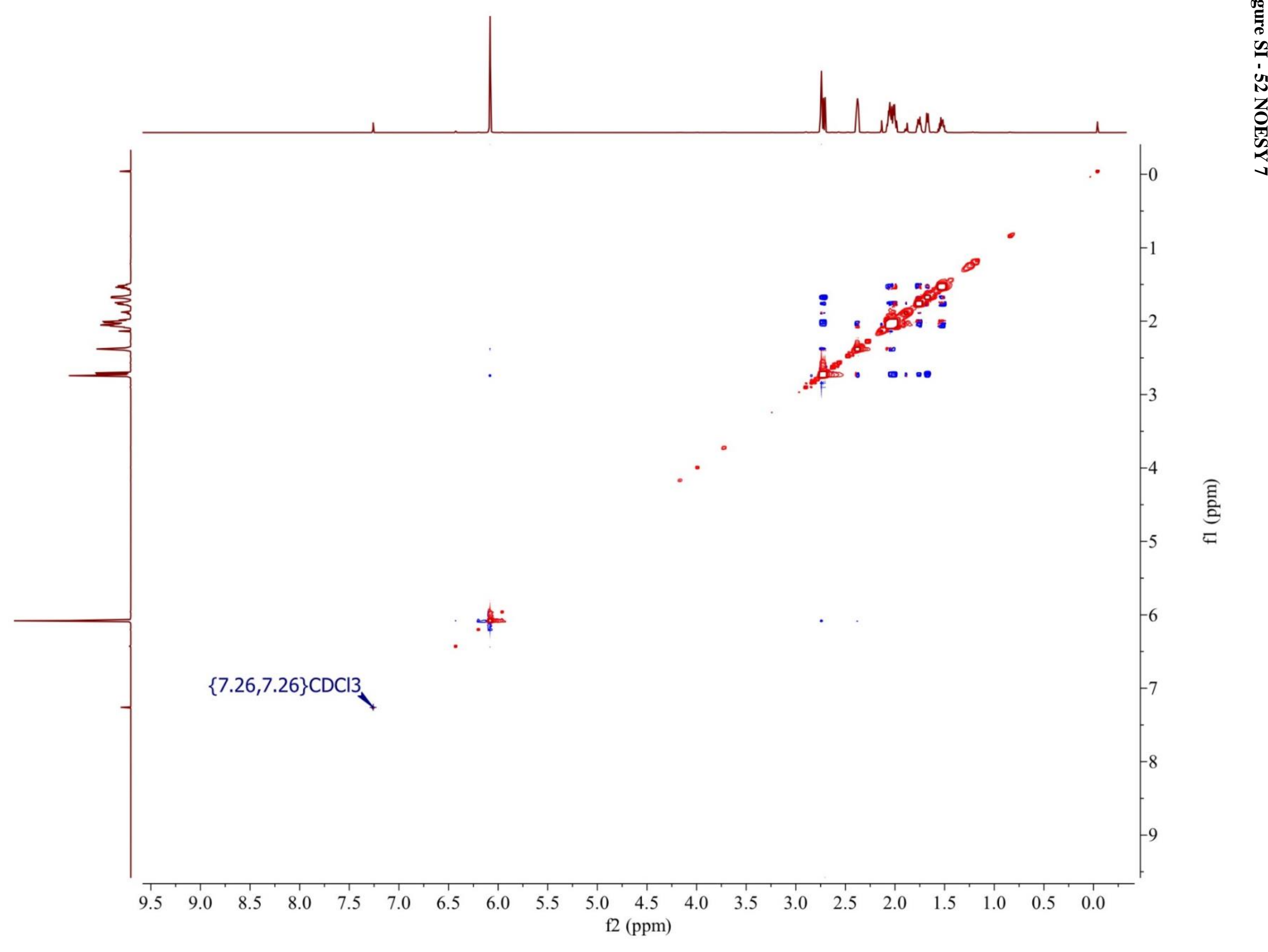


Table SI - 10 Crystallographic Data 7

\begin{tabular}{|c|c|}
\hline Empirical formula & $\mathrm{C}_{11} \mathrm{H}_{14} \mathrm{O}$ \\
\hline Formula weight & $162.23 \mathrm{~g} / \mathrm{mol}$ \\
\hline Temperature & $173 \mathrm{~K}$ \\
\hline Wavelength & $1.54184 \AA$ \\
\hline Crystal system & orthorhombic \\
\hline Space group & $\mathrm{Pccn}$ \\
\hline Unit cell dimensions & $\begin{array}{l}\mathrm{a}=20.0831(9) \AA, \alpha=90^{\circ} \\
\mathrm{b}=7.6632(3) \AA, \beta=90^{\circ} \\
\mathrm{c}=10.9517(5) \AA, \gamma=90^{\circ}\end{array}$ \\
\hline Volume & $1685.48 \AA^{3}$ \\
\hline $\mathbf{Z}$ & 8 \\
\hline Density (calculated) & $1.279 \mathrm{~g} / \mathrm{cm}^{3}$ \\
\hline Absorption coefficient & $0.618 \mathrm{~mm}^{-1}$ \\
\hline $\mathbf{F}(\mathbf{0 0 0})$ & 704 \\
\hline$\theta_{\max }$ & $70.04^{\circ}$ \\
\hline Crystal size $\left(\mathrm{mm}^{3}\right)$ & $0.242 \times 0.345 \times 0.387 \mathrm{~mm}$ \\
\hline Exposure time & $13.68 \mathrm{hr}$ \\
\hline $\begin{array}{l}\text { Reflections } \\
\text { collected }\end{array}$ & $\begin{array}{l}12917 \\
{\left[\mathrm{R}_{\text {int }}=1.85 \%\right]}\end{array}$ \\
\hline Independent reflections & 1589 \\
\hline Completeness to $\theta \max$ & $98.9 \%$ \\
\hline Min./max. transmission & 0.7960 \\
\hline Refinement method & Full matrix least squares on $\mathrm{F} 2$ \\
\hline Absorption correction & Numerical \\
\hline Goodness-of-fit & 1.098 \\
\hline $\begin{array}{l}\text { Final } R \text { indices } \\
{[I>2 \theta(I)]}\end{array}$ & $5.24 \%$ \\
\hline $\mathbf{R}$ indices (all) & $13.21 \%$ \\
\hline $\begin{array}{l}\text { Largest diff. peak } \\
\text { and hole }\end{array}$ & $0.235,-0.529 \mathrm{e}^{-} / \AA^{3}$ \\
\hline
\end{tabular}

Figure SI - 53 X-Ray crystal structure 7. Atomic displacement ellipsoids generated at $50 \%$ probability Recrystallized from a minimum of hot hexanes.
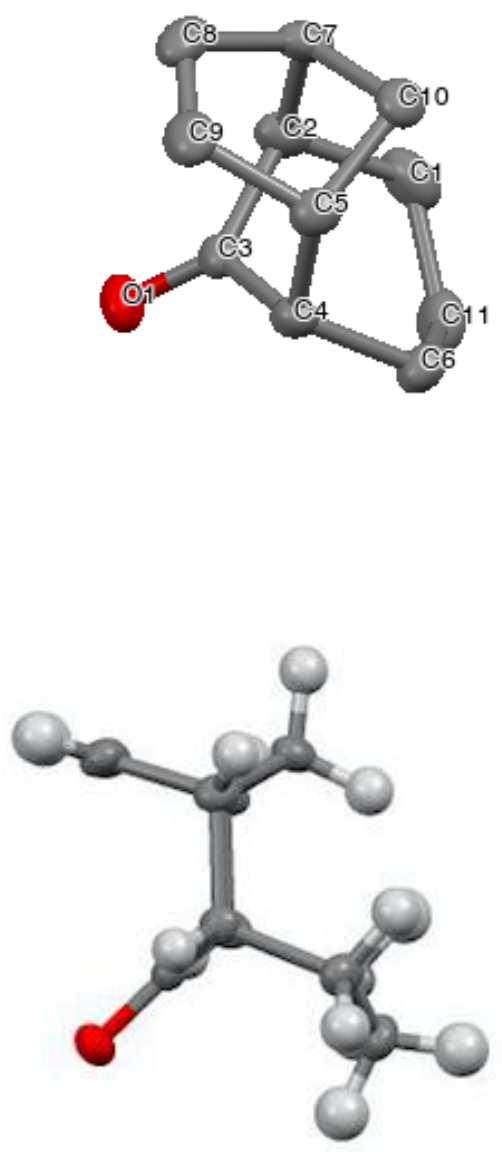
Table SI - 11 Dihedral Angles $\left({ }^{\circ}\right) 7$

\begin{tabular}{|c|c|c|c|c|}
\hline Number & Atom1 & Atom2 & Atom3 & Angle \\
\hline 1 & $\mathrm{H} 1$ & $\mathrm{C} 1$ & H11 & 107.9 \\
\hline 2 & $\mathrm{H} 1$ & $\mathrm{C} 1$ & $\mathrm{C} 2$ & 109.0 \\
\hline 3 & $\mathrm{H} 1$ & $\mathrm{C} 1$ & C11 & 109.0 \\
\hline 4 & H11 & $\mathrm{C} 1$ & $\mathrm{C} 2$ & 109.0 \\
\hline 5 & H11 & $\mathrm{C} 1$ & C11 & 109.0 \\
\hline 6 & $\mathrm{C} 2$ & $\mathrm{C} 1$ & C11 & $112.9(1)$ \\
\hline 7 & $\mathrm{C} 1$ & $\mathrm{C} 2$ & H12 & 109.7 \\
\hline 8 & $\mathrm{C} 1$ & $\mathrm{C} 2$ & $\mathrm{C} 3$ & $106.2(1)$ \\
\hline 9 & $\mathrm{C} 1$ & $\mathrm{C} 2$ & $\mathrm{C} 7$ & 113.7(1) \\
\hline 10 & $\mathrm{H} 12$ & $\mathrm{C} 2$ & $\mathrm{C} 3$ & 109.7 \\
\hline 11 & $\mathrm{H} 12$ & $\mathrm{C} 2$ & C7 & 109.7 \\
\hline 12 & $\mathrm{C} 3$ & $\mathrm{C} 2$ & C7 & $107.9(1)$ \\
\hline 13 & $\mathrm{O} 1$ & $\mathrm{C} 3$ & $\mathrm{C} 2$ & $123.9(1)$ \\
\hline 14 & $\mathrm{O} 1$ & $\mathrm{C} 3$ & $\mathrm{C} 4$ & $123.8(1)$ \\
\hline 15 & $\mathrm{C} 2$ & $\mathrm{C} 3$ & $\mathrm{C} 4$ & $112.2(1)$ \\
\hline 16 & $\mathrm{C} 3$ & $\mathrm{C} 4$ & H13 & 109.6 \\
\hline 17 & $\mathrm{C} 3$ & $\mathrm{C} 4$ & $\mathrm{C} 5$ & $107.8(1)$ \\
\hline 18 & $\mathrm{C} 3$ & $\mathrm{C} 4$ & C6 & $106.5(1)$ \\
\hline 19 & $\mathrm{H} 13$ & $\mathrm{C} 4$ & C5 & 109.7 \\
\hline 20 & H13 & $\mathrm{C} 4$ & C6 & 109.7 \\
\hline 21 & $\mathrm{C} 5$ & $\mathrm{C} 4$ & C6 & $113.5(1)$ \\
\hline 22 & $\mathrm{C} 4$ & $\mathrm{C} 5$ & $\mathrm{H} 2$ & 112.6 \\
\hline 23 & $\mathrm{C} 4$ & $\mathrm{C} 5$ & C9 & $108.6(1)$ \\
\hline 24 & $\mathrm{C} 4$ & $\mathrm{C} 5$ & $\mathrm{C} 10$ & 109.9(1) \\
\hline 25 & $\mathrm{H} 2$ & $\mathrm{C} 5$ & C9 & 112.6 \\
\hline 26 & $\mathrm{H} 2$ & $\mathrm{C} 5$ & $\mathrm{C} 10$ & 112.5 \\
\hline 27 & C9 & $\mathrm{C} 5$ & $\mathrm{C} 10$ & $99.9(1)$ \\
\hline 28 & $\mathrm{C} 4$ & C6 & H14 & 109.1 \\
\hline 29 & $\mathrm{C} 4$ & C6 & $\mathrm{H} 3$ & 109.1 \\
\hline 30 & $\mathrm{C} 4$ & C6 & $\mathrm{C} 11$ & $112.5(1)$ \\
\hline 31 & H14 & C6 & $\mathrm{H} 3$ & 107.8 \\
\hline 32 & $\mathrm{H} 14$ & C6 & C11 & 109.1 \\
\hline 33 & $\mathrm{H} 3$ & C6 & C11 & 109.2 \\
\hline 34 & $\mathrm{C} 2$ & $\mathrm{C} 7$ & $\mathrm{H} 8$ & 112.7 \\
\hline 35 & $\mathrm{C} 2$ & $\mathrm{C} 7$ & $\mathrm{C} 8$ & $108.8(1)$ \\
\hline 36 & $\mathrm{C} 2$ & $\mathrm{C} 7$ & $\mathrm{C} 10$ & $109.4(1)$ \\
\hline 37 & $\mathrm{H} 8$ & $\mathrm{C} 7$ & $\mathrm{C} 8$ & 112.8 \\
\hline 38 & $\mathrm{H} 8$ & $\mathrm{C} 7$ & $\mathrm{C} 10$ & 112.8 \\
\hline 39 & $\mathrm{C} 8$ & $\mathrm{C} 7$ & $\mathrm{C} 10$ & $99.6(1)$ \\
\hline
\end{tabular}




\begin{tabular}{|c|c|c|c|c|}
\hline 40 & $\mathrm{C} 7$ & $\mathrm{C} 8$ & $\mathrm{H} 7$ & 125.3 \\
\hline 41 & $\mathrm{C} 7$ & $\mathrm{C} 8$ & C9 & 109.4(1) \\
\hline 42 & H7 & $\mathrm{C} 8$ & C9 & 125.4 \\
\hline 43 & C5 & C9 & $\mathrm{C} 8$ & 109.7(1) \\
\hline 44 & C5 & C9 & H6 & 125.2 \\
\hline 45 & $\mathrm{C} 8$ & C9 & H6 & 125.1 \\
\hline 46 & C5 & $\mathrm{C} 10$ & C7 & $99.4(1)$ \\
\hline 47 & $\mathrm{C} 5$ & $\mathrm{C} 10$ & H5 & 111.9 \\
\hline 48 & $\mathrm{C} 5$ & $\mathrm{C} 10$ & $\mathrm{H} 4$ & 111.9 \\
\hline 49 & C7 & $\mathrm{C} 10$ & H5 & 112.0 \\
\hline 50 & C7 & $\mathrm{C} 10$ & $\mathrm{H} 4$ & 111.9 \\
\hline 51 & H5 & $\mathrm{C} 10$ & H4 & 109.6 \\
\hline 52 & $\mathrm{C} 1$ & $\mathrm{C} 11$ & C6 & $115.4(1)$ \\
\hline 53 & $\mathrm{C} 1$ & C11 & $\mathrm{H} 10$ & 108.4 \\
\hline 54 & $\mathrm{C} 1$ & C11 & H9 & 108.4 \\
\hline 55 & C6 & C11 & H10 & 108.4 \\
\hline 56 & C6 & C11 & H9 & 108.4 \\
\hline 57 & H10 & C11 & H9 & 107.5 \\
\hline
\end{tabular}


Table SI - 12 Bond Length (̊̊) 7

\begin{tabular}{|c|c|c|c|}
\hline Number & Atom1 & Atom2 & Length \\
\hline 1 & $\mathrm{O} 1$ & $\mathrm{C} 3$ & $1.214(2)$ \\
\hline 2 & $\mathrm{C} 1$ & $\mathrm{H} 1$ & 0.989 \\
\hline 3 & $\mathrm{C} 1$ & H11 & 0.990 \\
\hline 4 & $\mathrm{C} 1$ & $\mathrm{C} 2$ & $1.555(2)$ \\
\hline 5 & $\mathrm{C} 1$ & C11 & $1.534(2)$ \\
\hline 6 & $\mathrm{C} 2$ & H12 & 0.999 \\
\hline 7 & $\mathrm{C} 2$ & $\mathrm{C} 3$ & $1.511(2)$ \\
\hline 8 & $\mathrm{C} 2$ & $\mathrm{C} 7$ & $1.564(2)$ \\
\hline 9 & $\mathrm{C} 3$ & $\mathrm{C} 4$ & $1.509(2)$ \\
\hline 10 & C4 & H13 & 0.999 \\
\hline 11 & $\mathrm{C} 4$ & $\mathrm{C} 5$ & $1.562(2)$ \\
\hline 12 & $\mathrm{C} 4$ & C6 & $1.554(2)$ \\
\hline 13 & C5 & $\mathrm{H} 2$ & 1.000 \\
\hline 14 & $\mathrm{C} 5$ & C9 & $1.517(2)$ \\
\hline 15 & C5 & $\mathrm{C} 10$ & $1.529(2)$ \\
\hline 16 & C6 & H14 & 0.990 \\
\hline 17 & C6 & H3 & 0.990 \\
\hline 18 & C6 & C11 & $1.540(2)$ \\
\hline 19 & $\mathrm{C} 7$ & H8 & 1.000 \\
\hline 20 & $\mathrm{C} 7$ & $\mathrm{C} 8$ & $1.522(2)$ \\
\hline 21 & C7 & $\mathrm{C} 10$ & $1.536(2)$ \\
\hline 22 & $\mathrm{C} 8$ & $\mathrm{H} 7$ & 0.950 \\
\hline 23 & $\mathrm{C} 8$ & C9 & $1.320(2)$ \\
\hline 24 & C9 & H6 & 0.949 \\
\hline 25 & $\mathrm{C} 10$ & H5 & 0.990 \\
\hline 26 & $\mathrm{C} 10$ & $\mathrm{H} 4$ & 0.990 \\
\hline 27 & $\mathrm{C} 11$ & $\mathrm{H} 10$ & 0.990 \\
\hline 28 & C11 & H9 & 0.990 \\
\hline
\end{tabular}




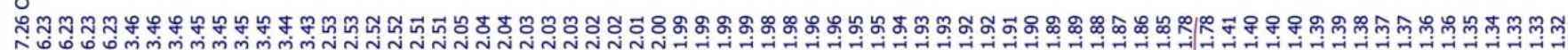

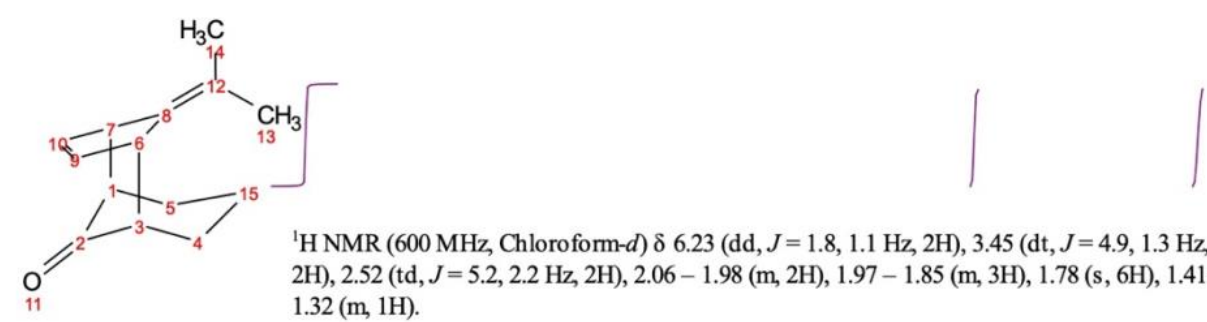
$2 \mathrm{H}), 2.52(\mathrm{td}, J=5.2,2.2 \mathrm{~Hz}, 2 \mathrm{H}), 2.06-1.98(\mathrm{~m}, 2 \mathrm{H}), 1.97-1.85(\mathrm{~m}, 3 \mathrm{H}), 1.78(\mathrm{~s}, 6 \mathrm{H}), 1.41$ $1.32(\mathrm{~m}, 1 \mathrm{H})$

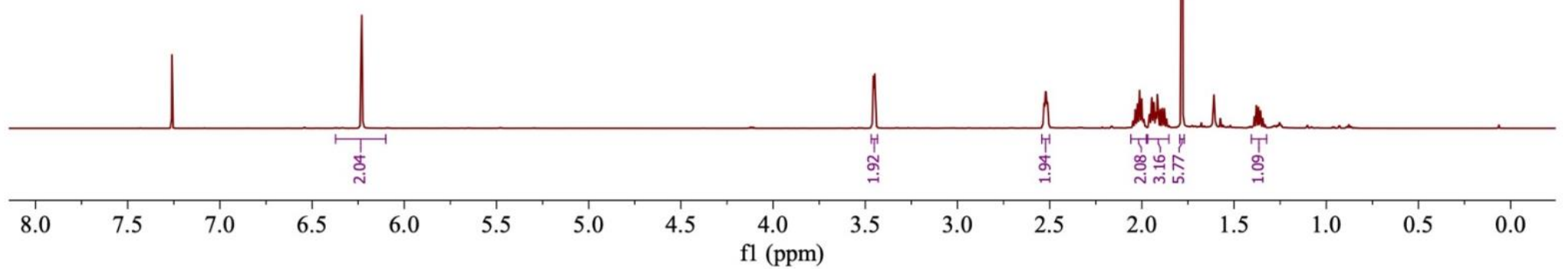




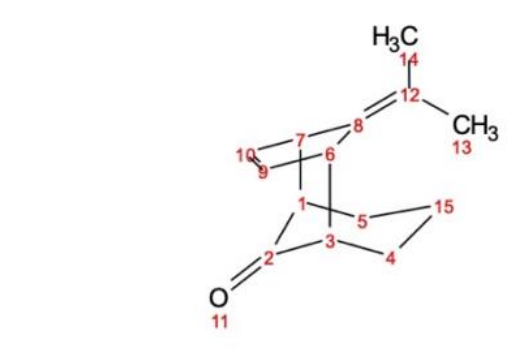




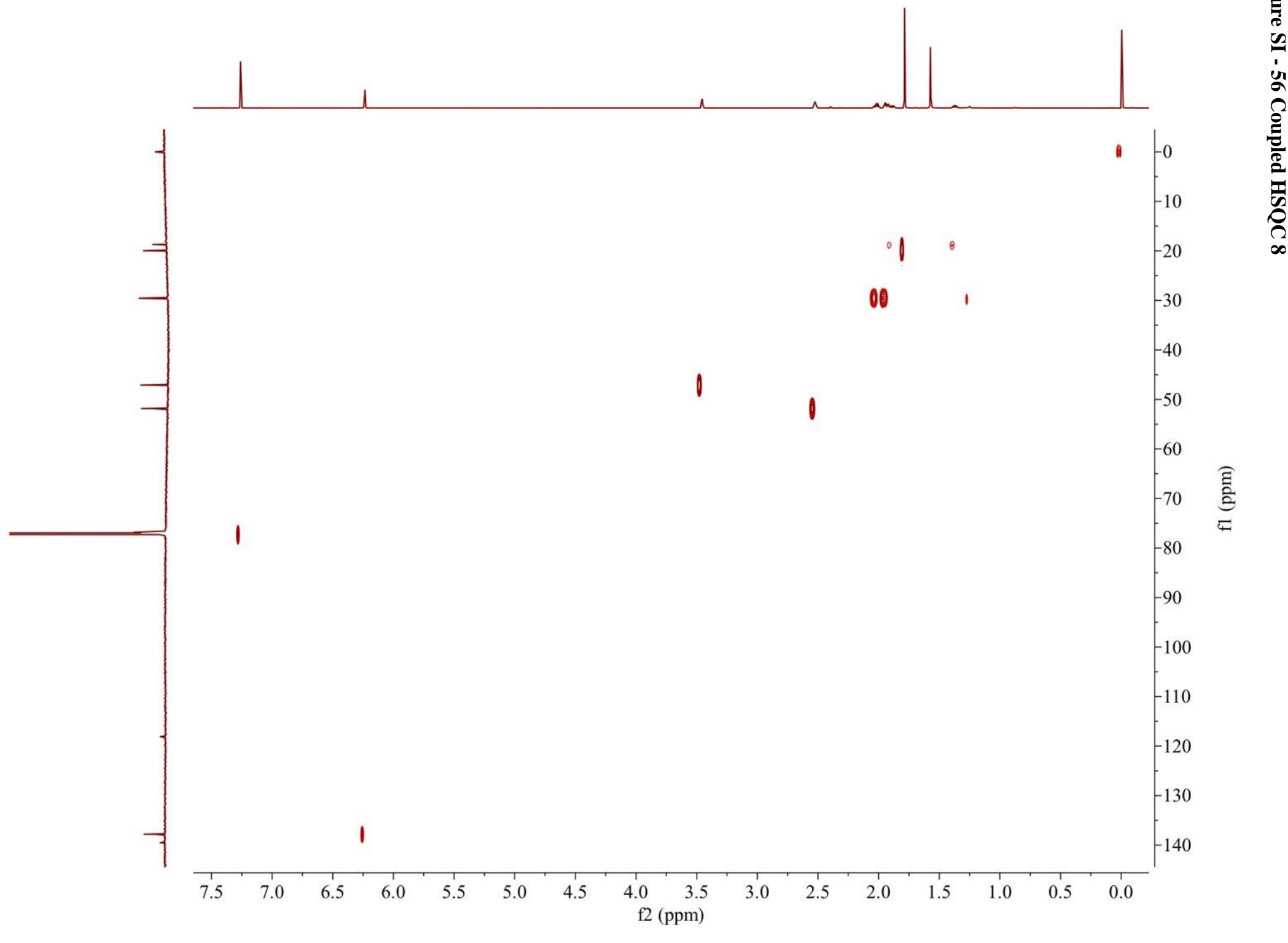




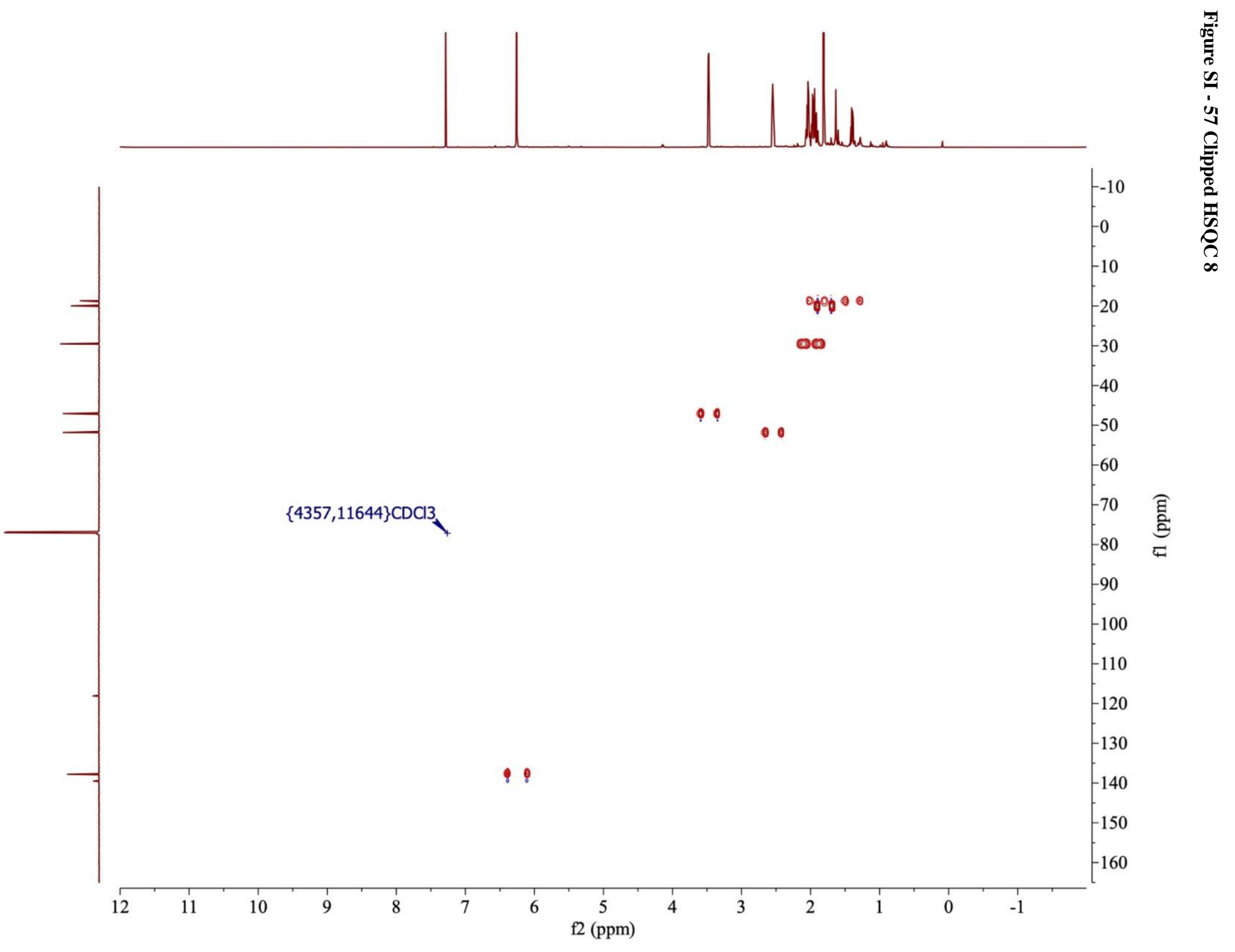




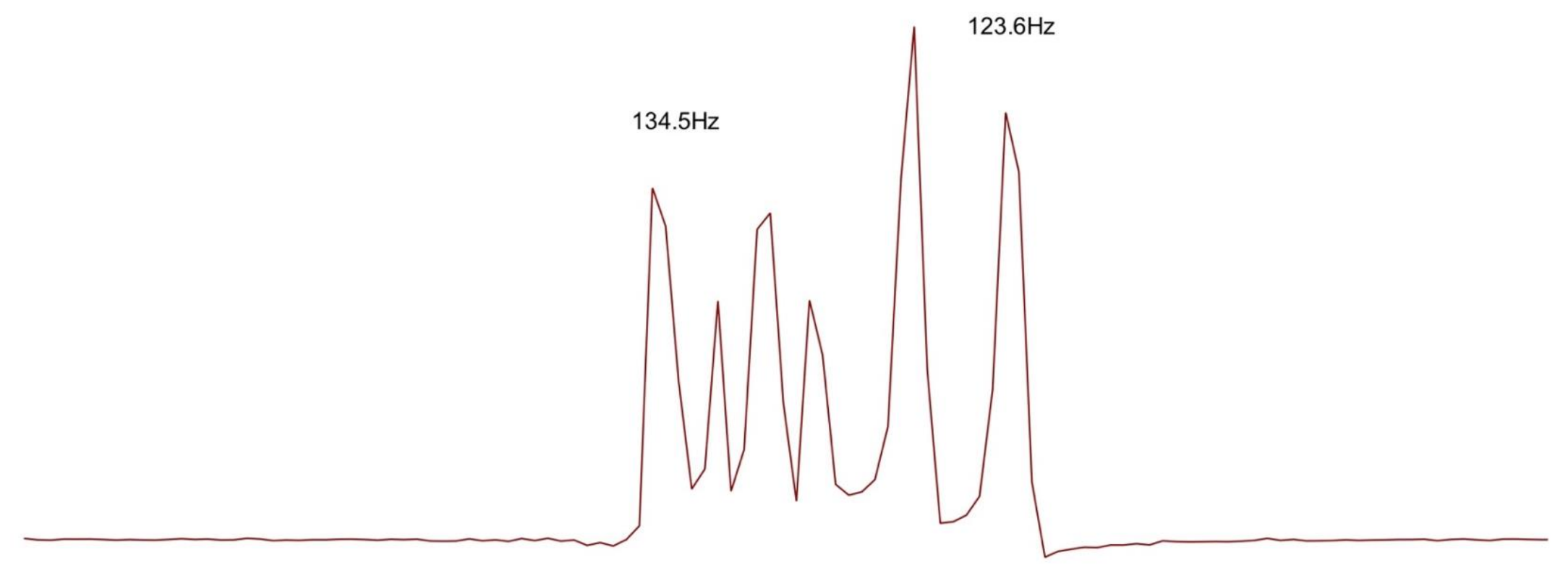




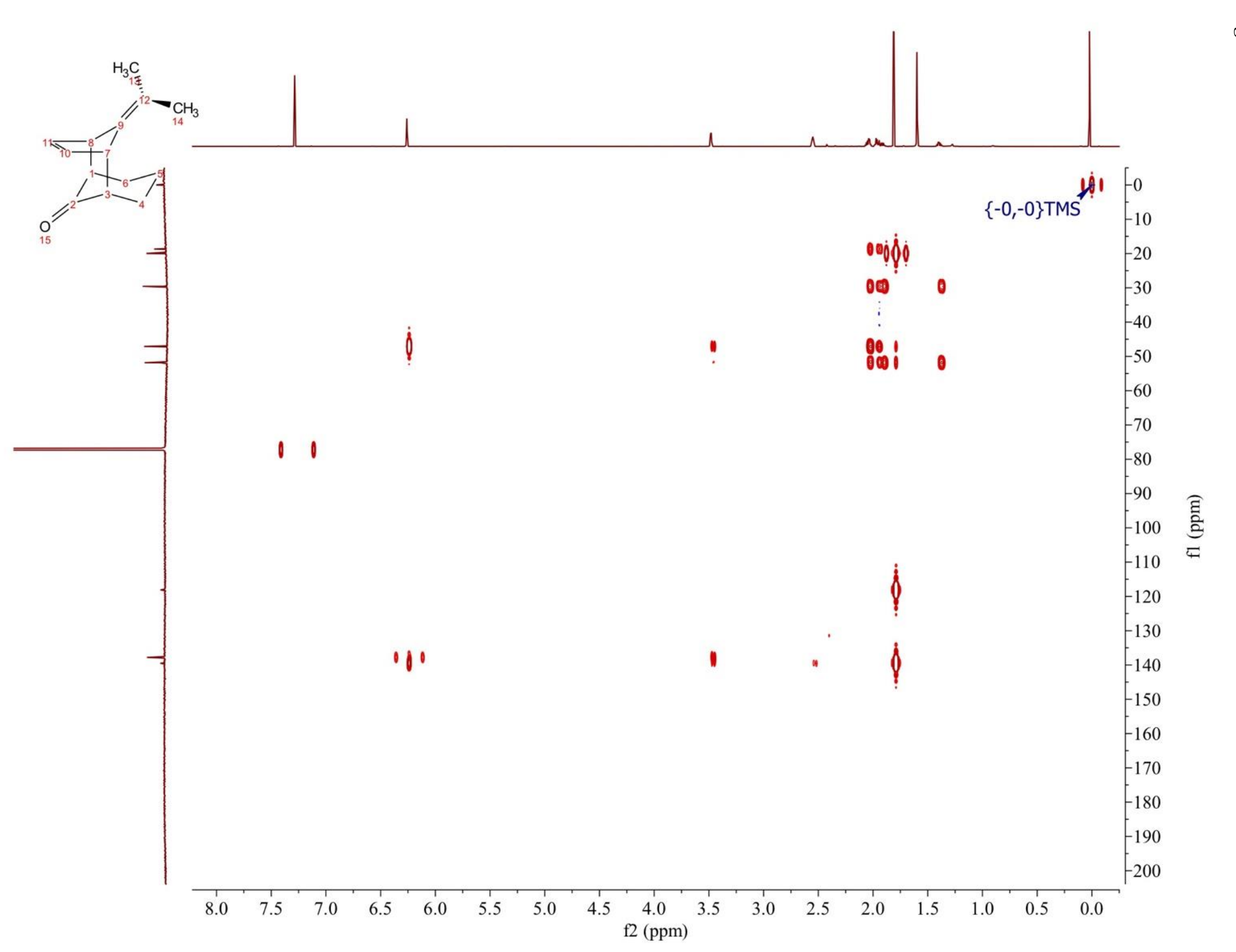




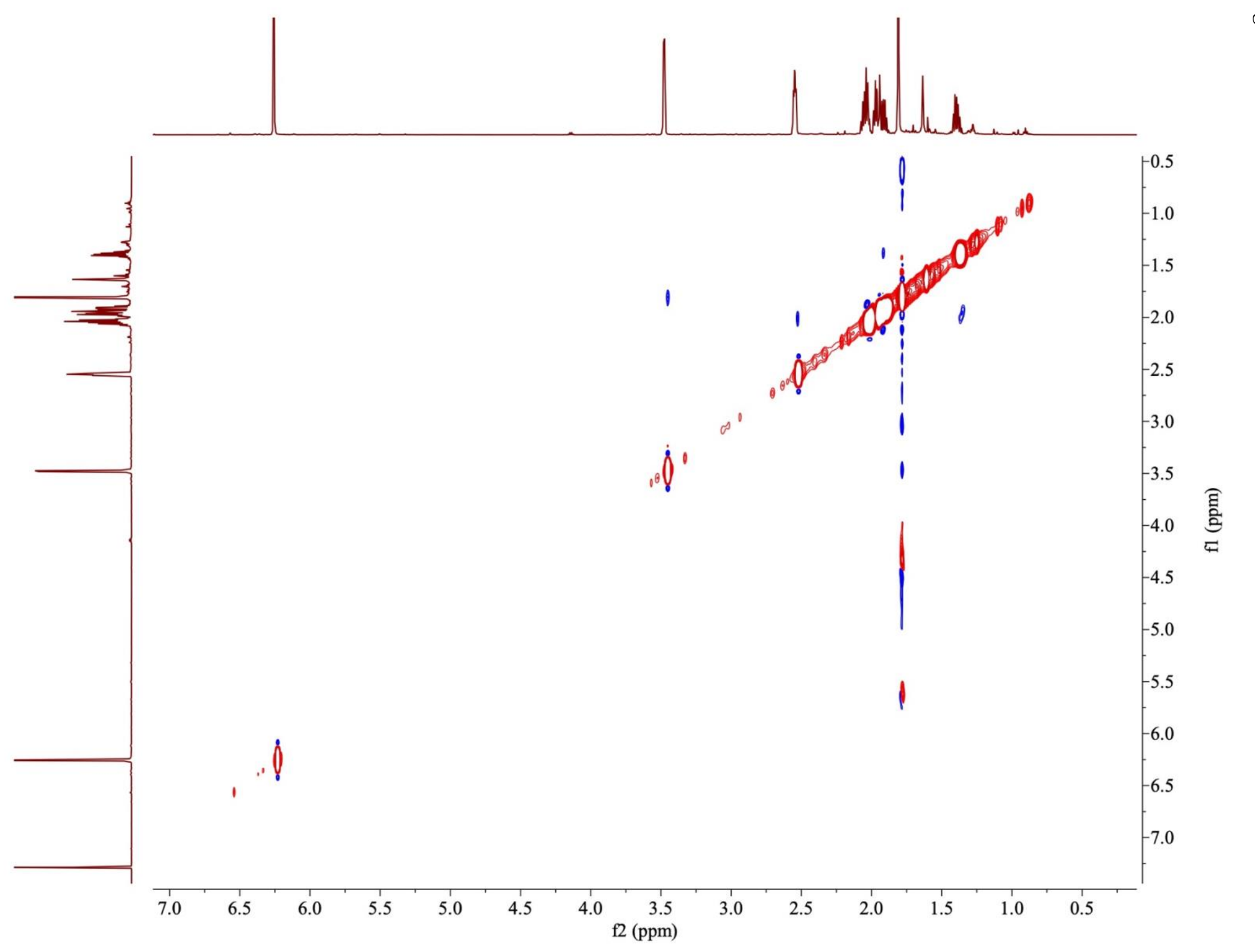

7
0
0
0
0
0
0
0
0
0
0
0
0
0 


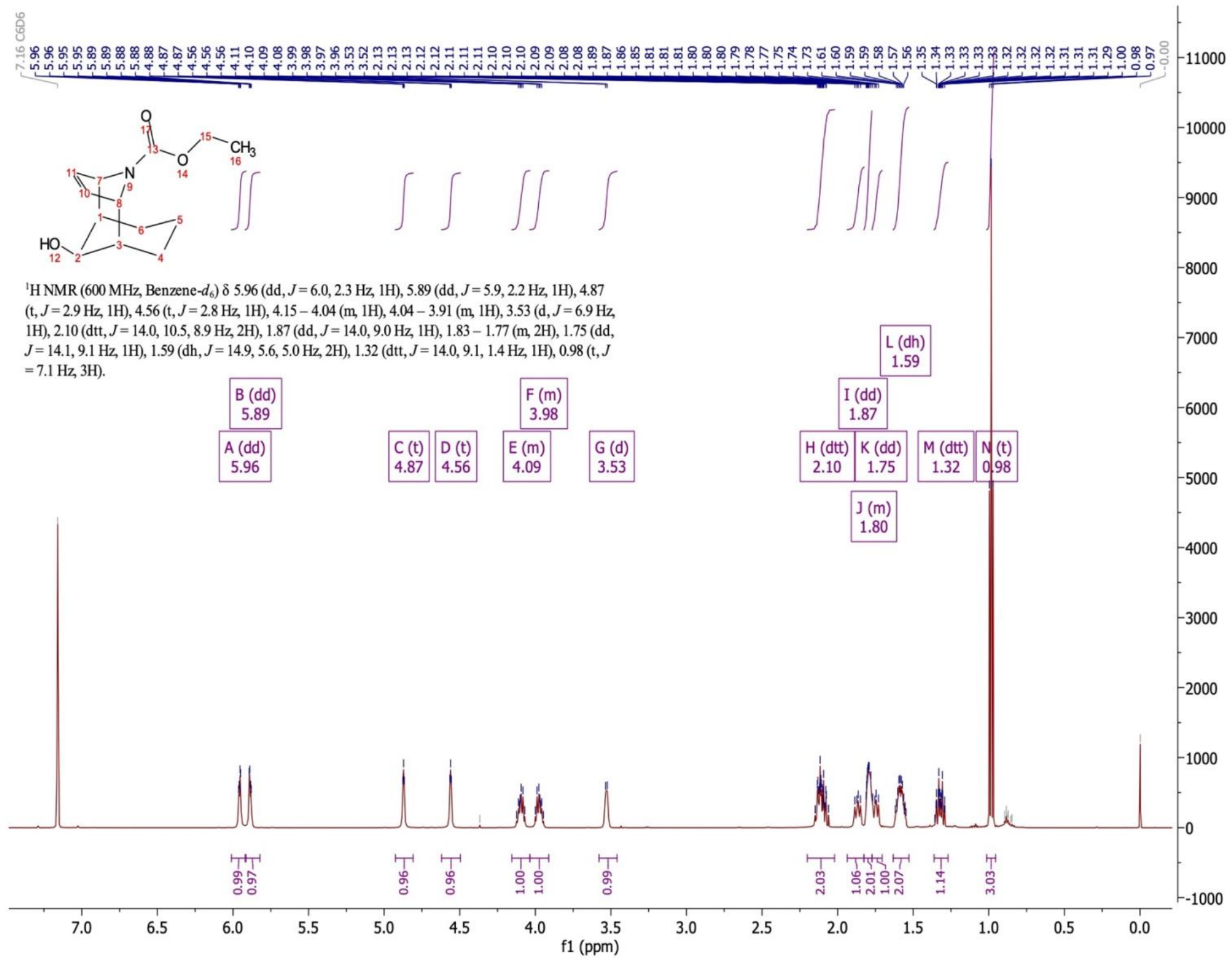

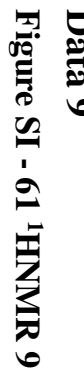




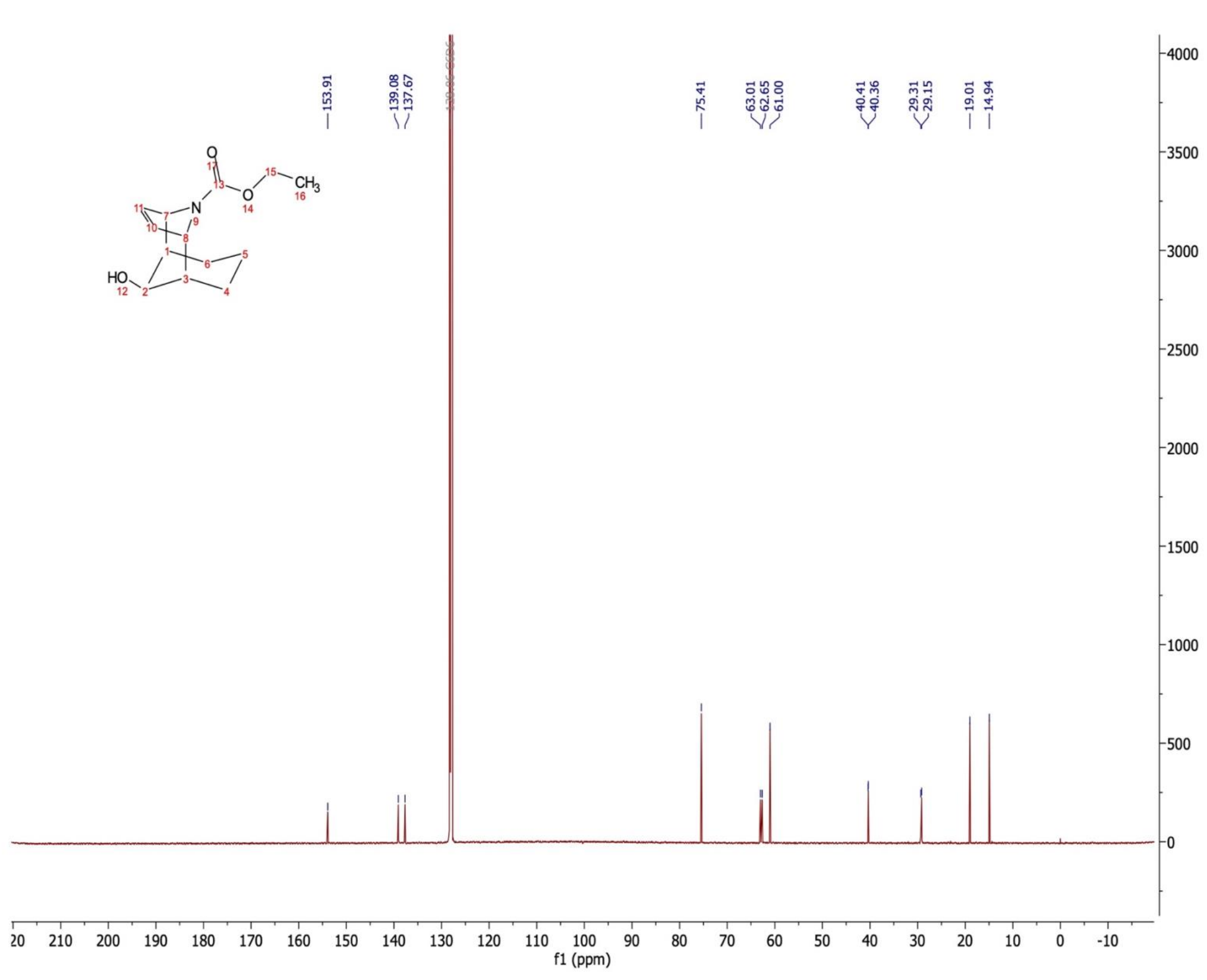




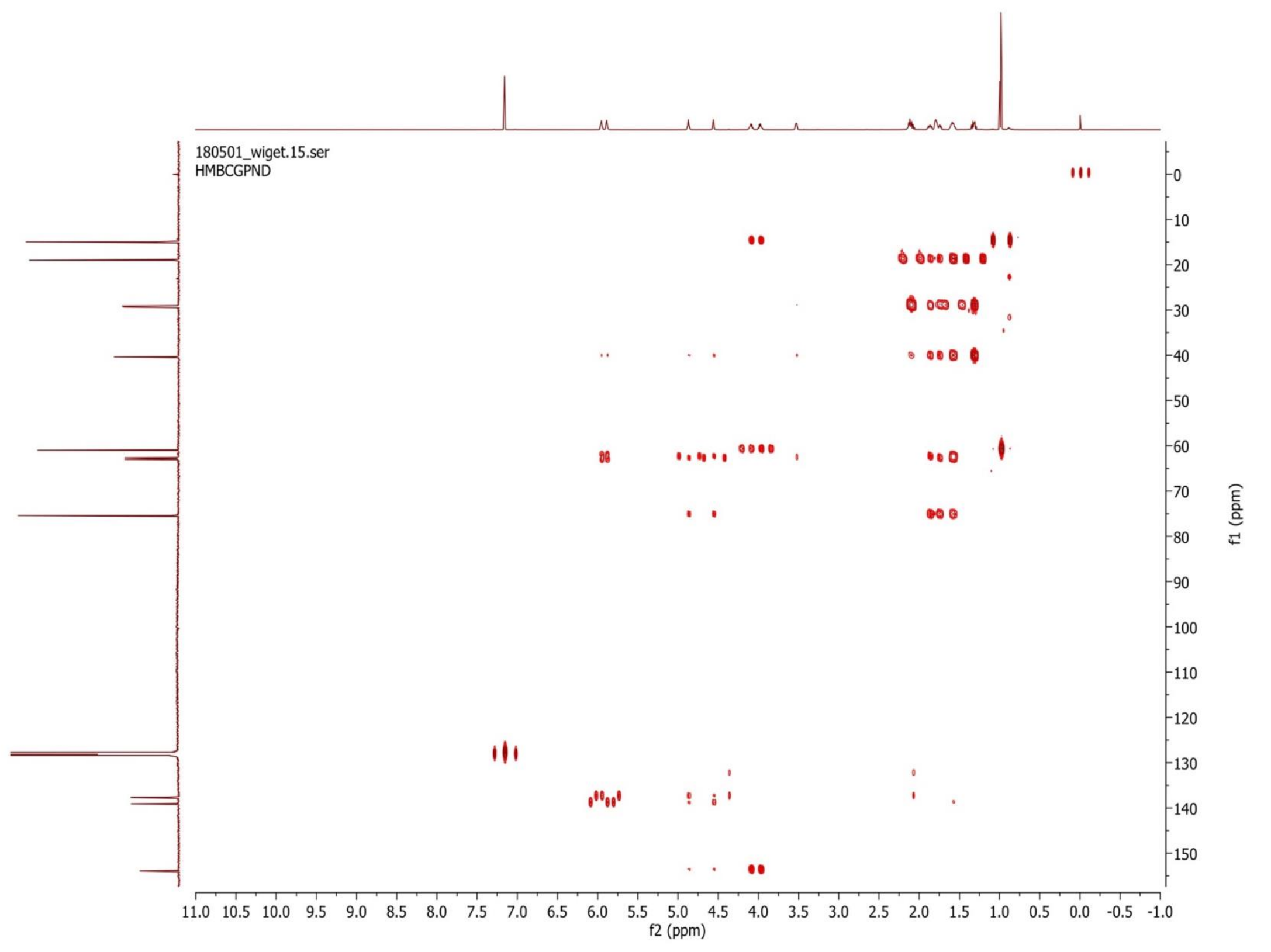

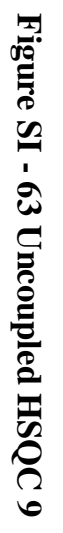




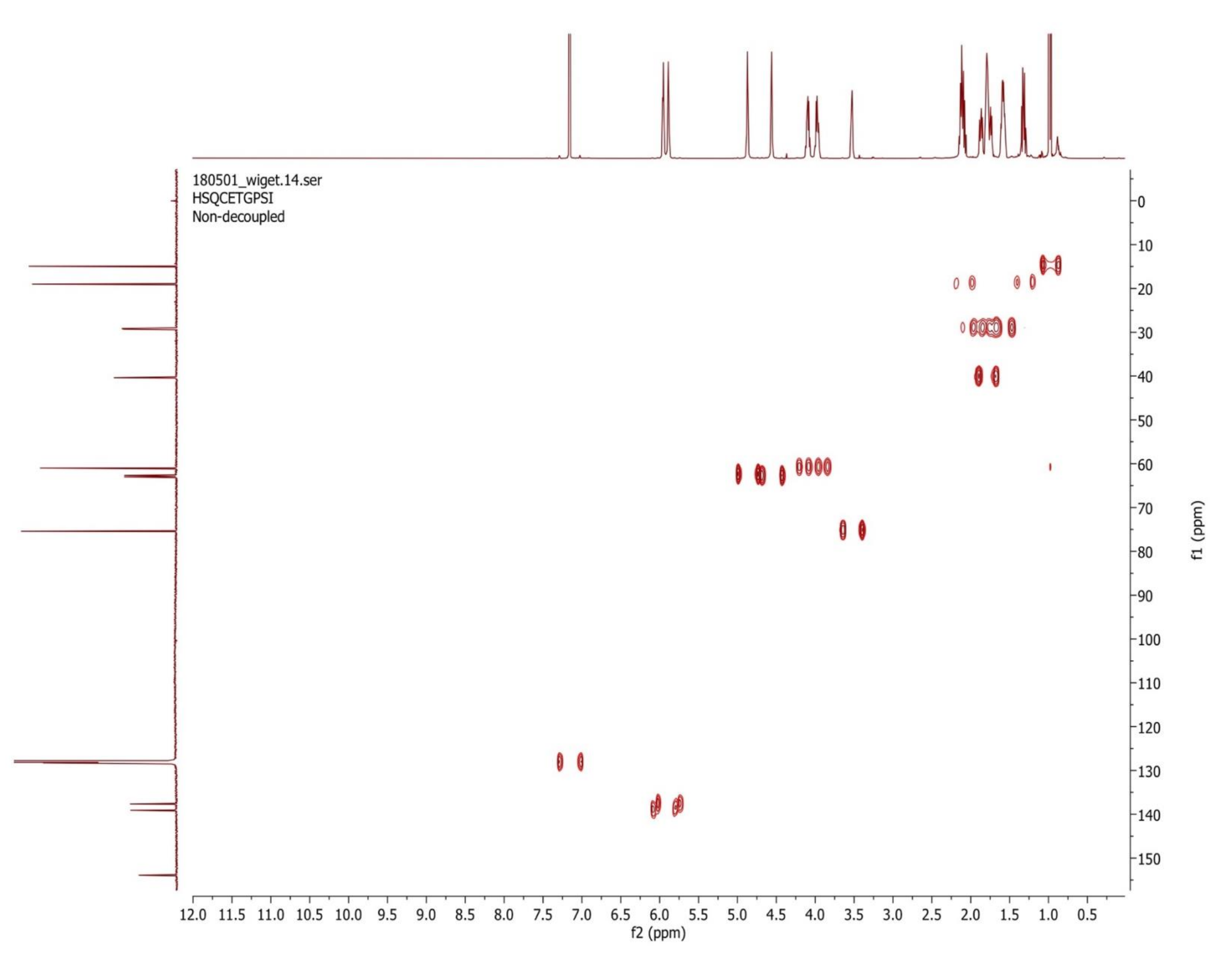

7
0
0
0
0
0
0
0
0
0
0
0
0
0
0
0
0
0
0 


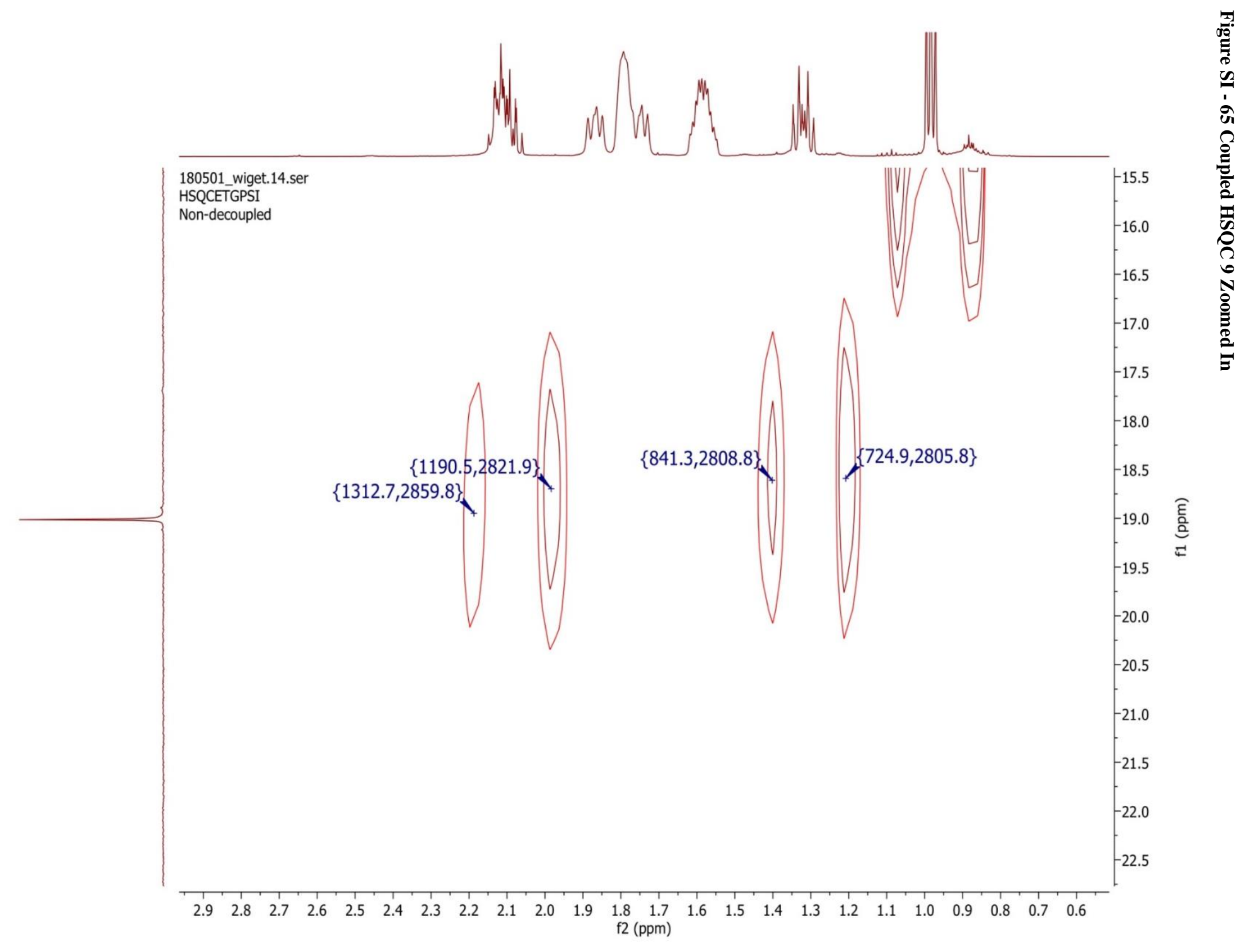




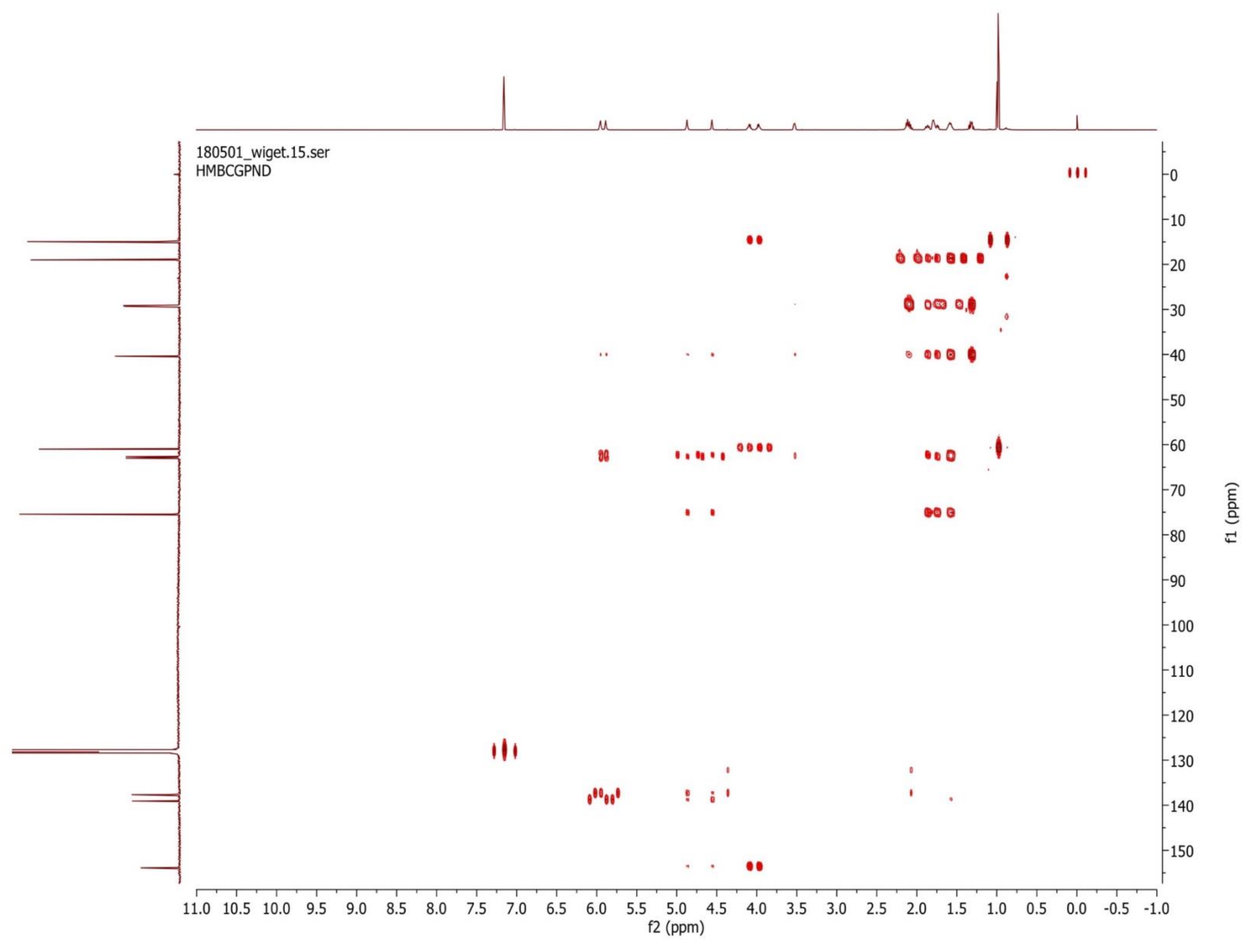




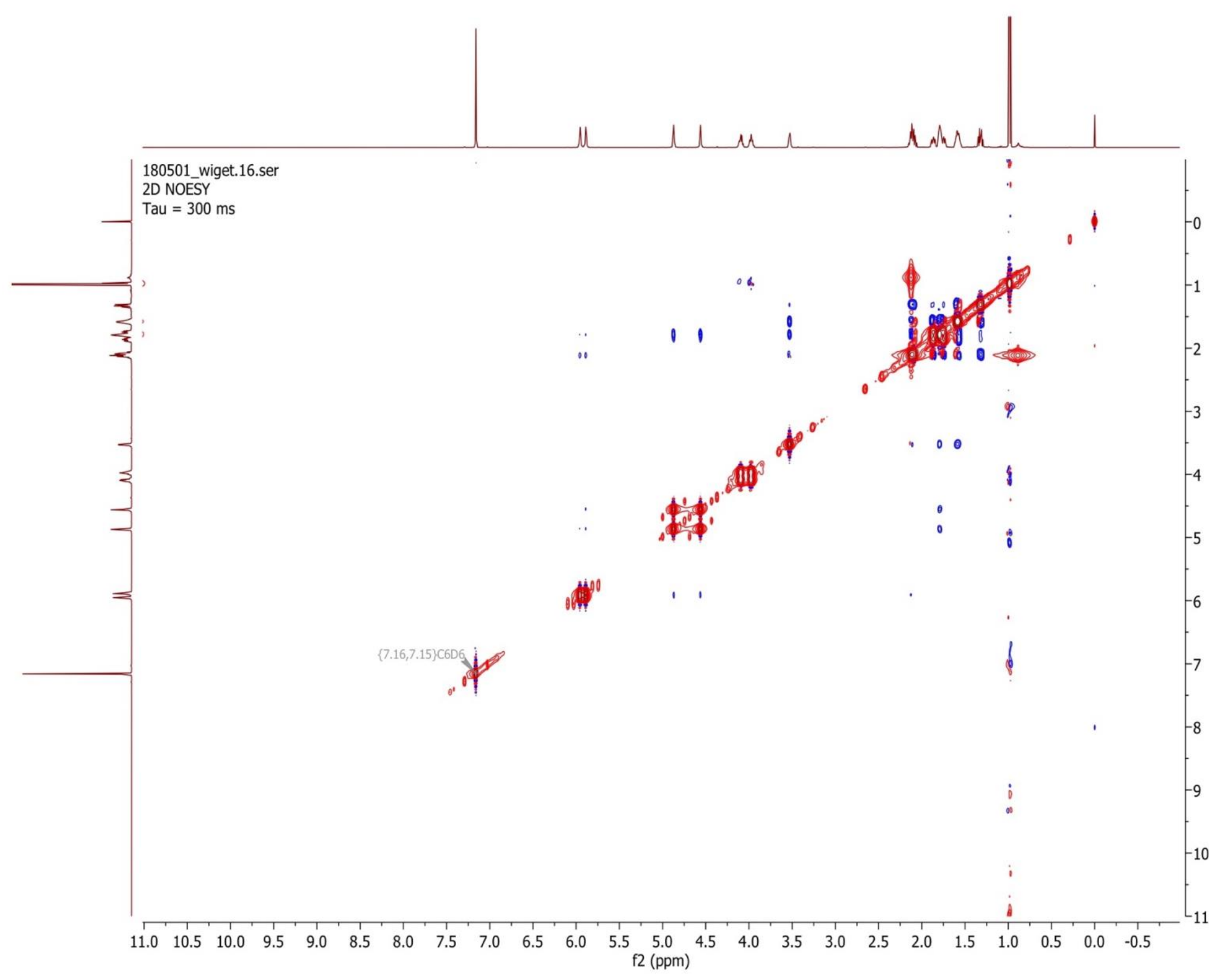

7
0
0
0
0
$\vdots$
$\vdots$
$z$
0
0
0
0
0 


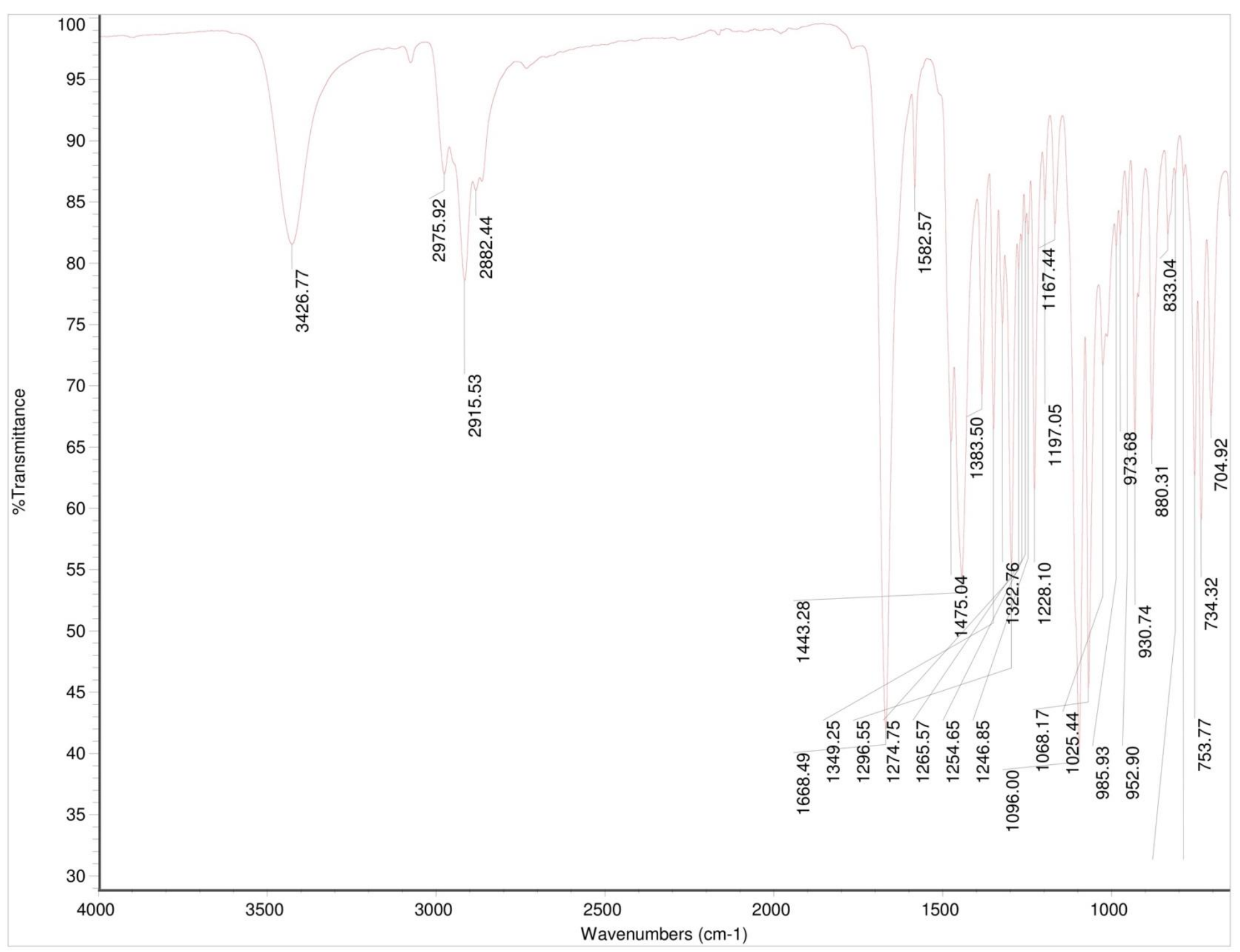




\section{RT: $7.71-8.88$}

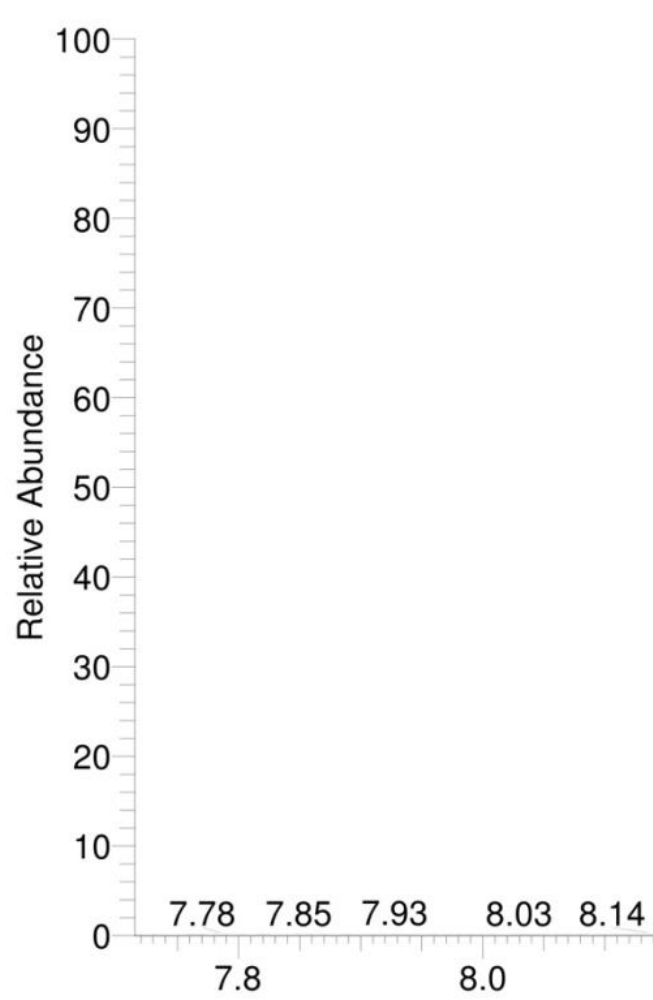

8.18
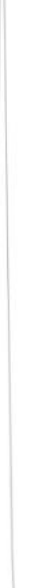

\begin{tabular}{rrrrrrr}
8.29 & 8.35 & 8.49 & 8.56 & 8.66 & 8.75 & 8.87 \\
\hline 8.2 & 8.4 & & 8.6 & & 8.8
\end{tabular}

RLJMPW-2_Concentrated01 \#2108-2117 RT: 8.17-8.20 AV: 10 NL: 2.54E6

T: $\{0,0\}+\mathrm{C}$ El Full ms [90.00-500.00]

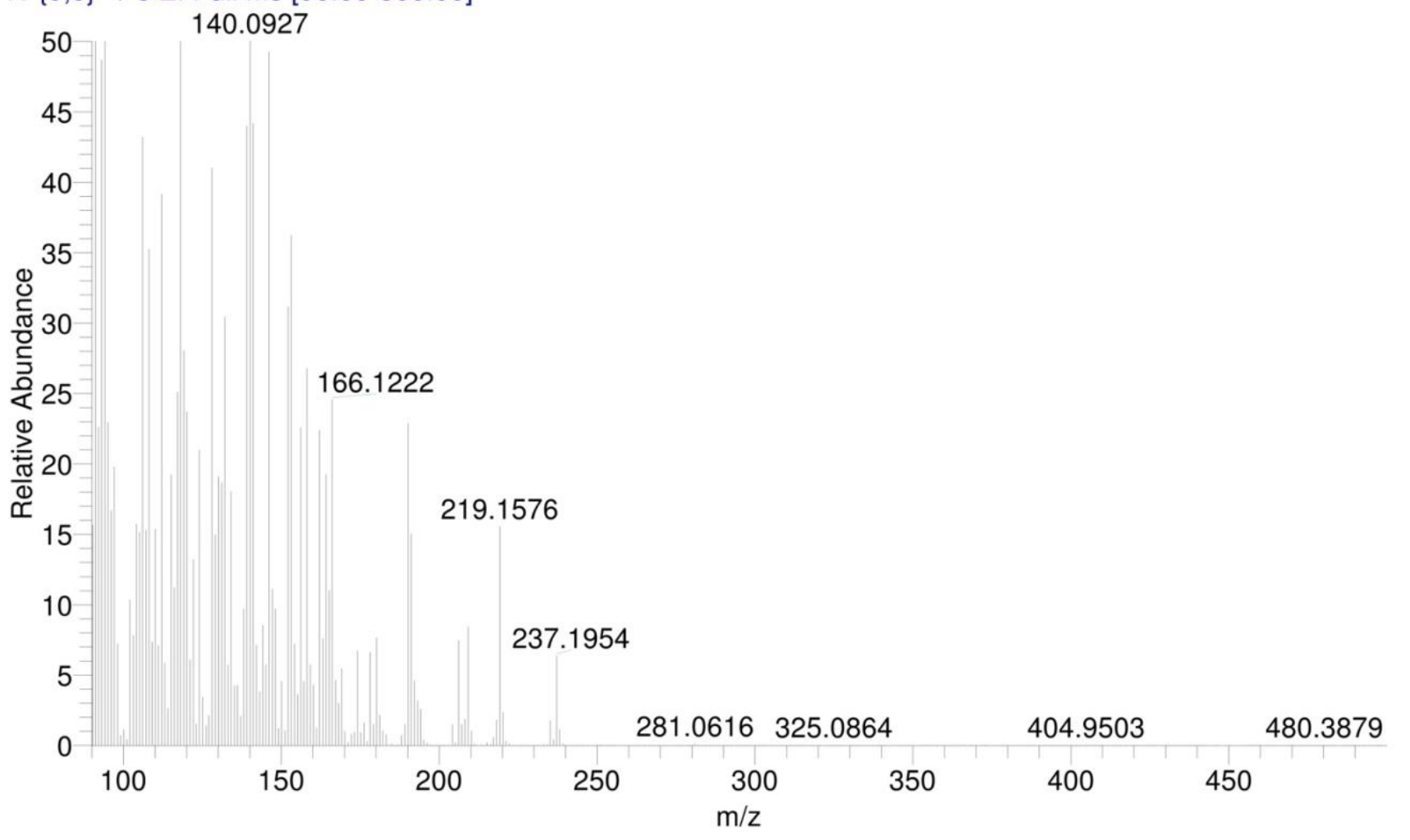


Figure SI - 70 High Resolution Mass Spectroscopy 9

Target Compound Screening Report

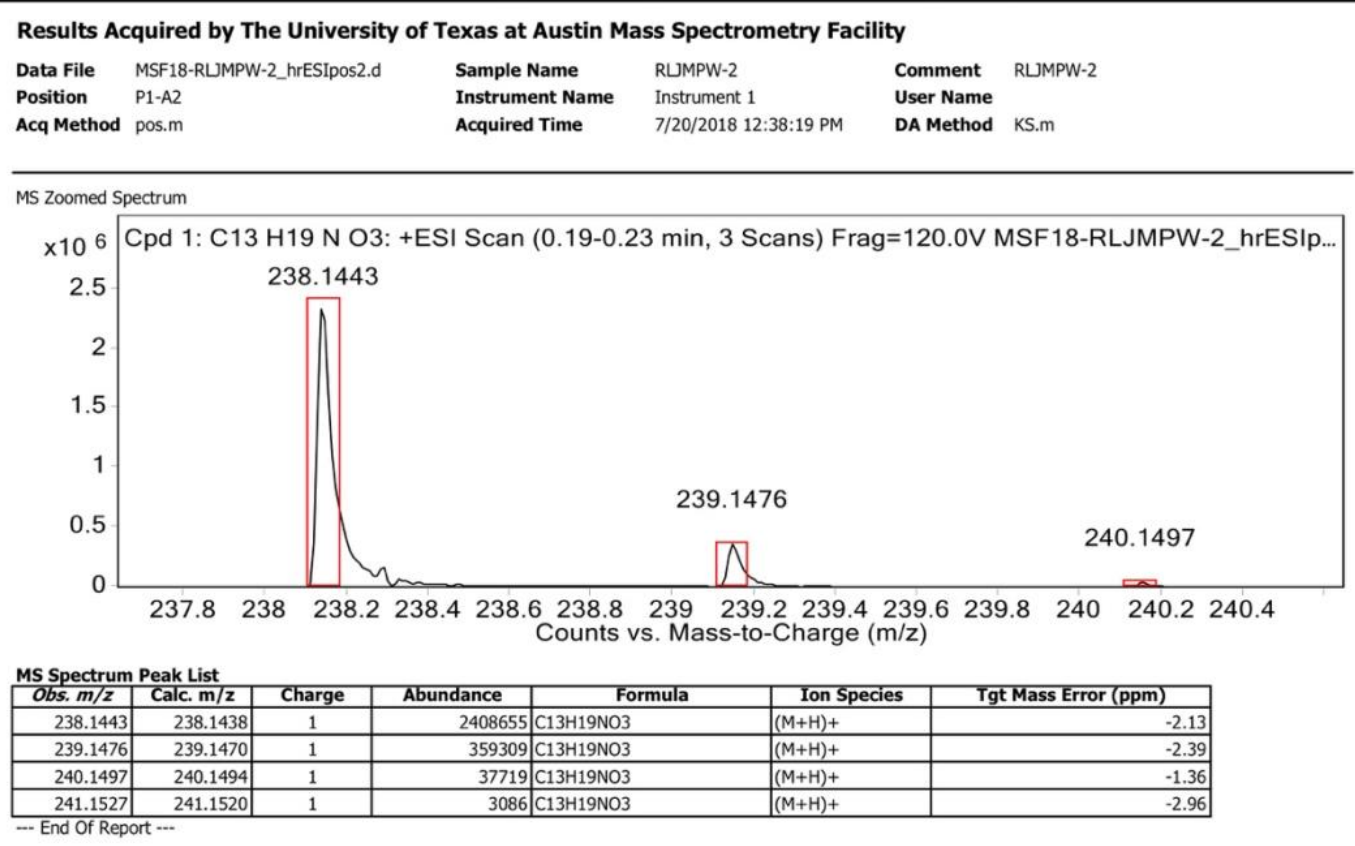


:

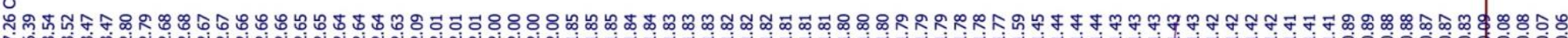
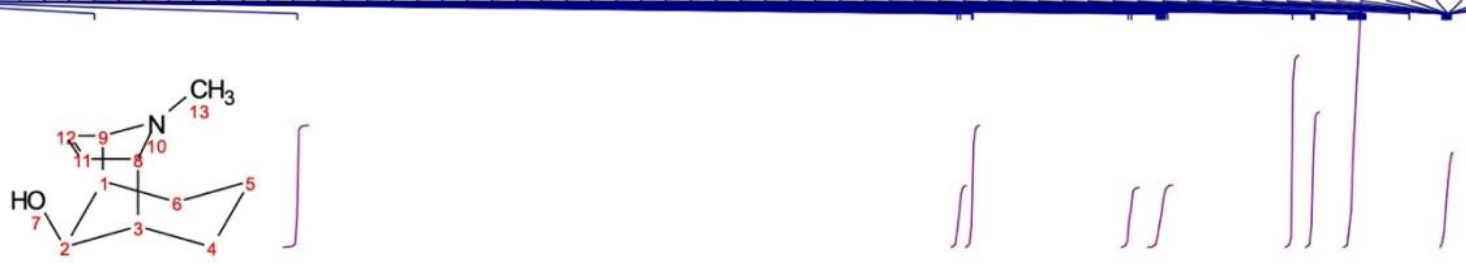

'H NMR ( $850 \mathrm{MHz}$, Chloroform- $d$ ) $\delta 6.39(\mathrm{~s}, 1 \mathrm{H}), 3.53$ (d, $J=11.1 \mathrm{~Hz}, 1 \mathrm{H}), 3.47$ (d, $J=4.1 \mathrm{~Hz}$ $2 \mathrm{H}), 2.79$ (d, $J=11.8 \mathrm{~Hz}, 1 \mathrm{H}), 2.66$ (dddd, $J=19.1,12.9,10.1,9.0 \mathrm{~Hz}, 1 \mathrm{H}$ ), 2.09 (s, 3H), 2.00 (tt, $J=4.0,2.0 \mathrm{~Hz}, 2 \mathrm{H}), 1.87-1.75$ (m, 4H), 1.43 (dtt, $J=14.8,8.4,1.8 \mathrm{~Hz}, 2 \mathrm{H})$.

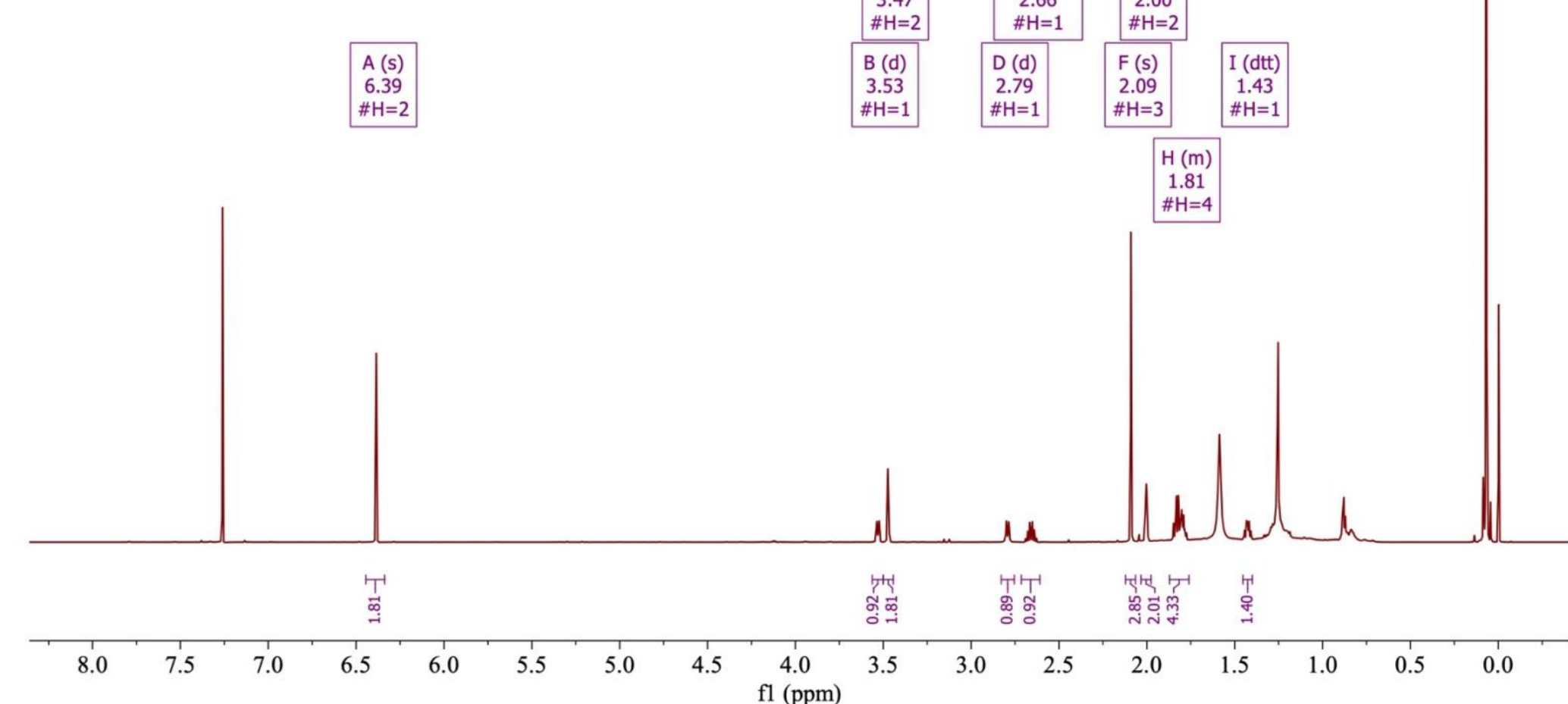




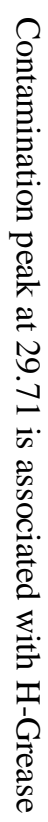
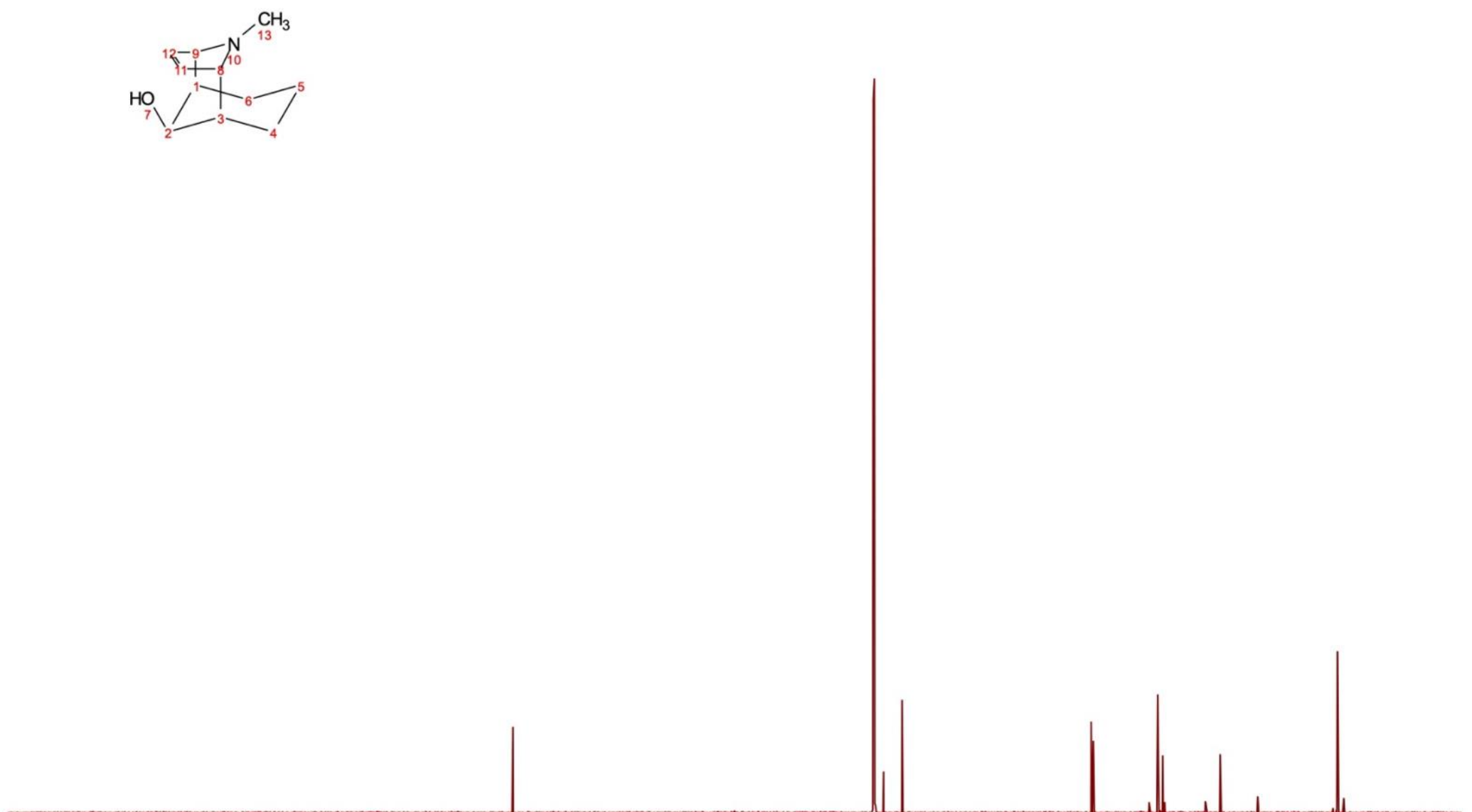

$\begin{array}{lllllllllllllllllllllll}210 & 200 & 190 & 180 & 170 & 160 & 150 & 140 & 130 & 120 & 110 & 100 & 90 & 80 & 70 & 60 & 50 & 40 & 30 & 20 & 10 & 0 & -10\end{array}$ f1 (ppm) 


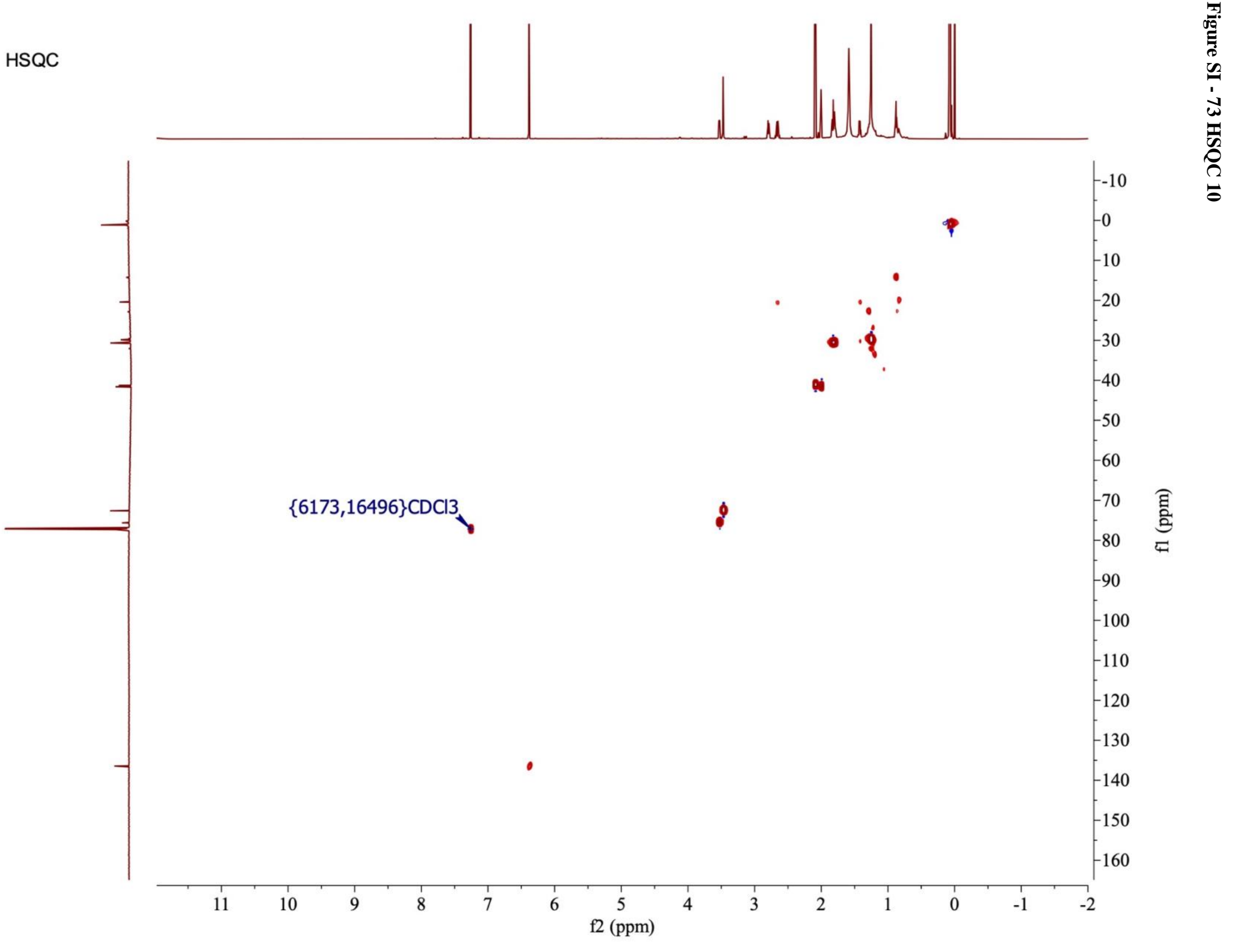




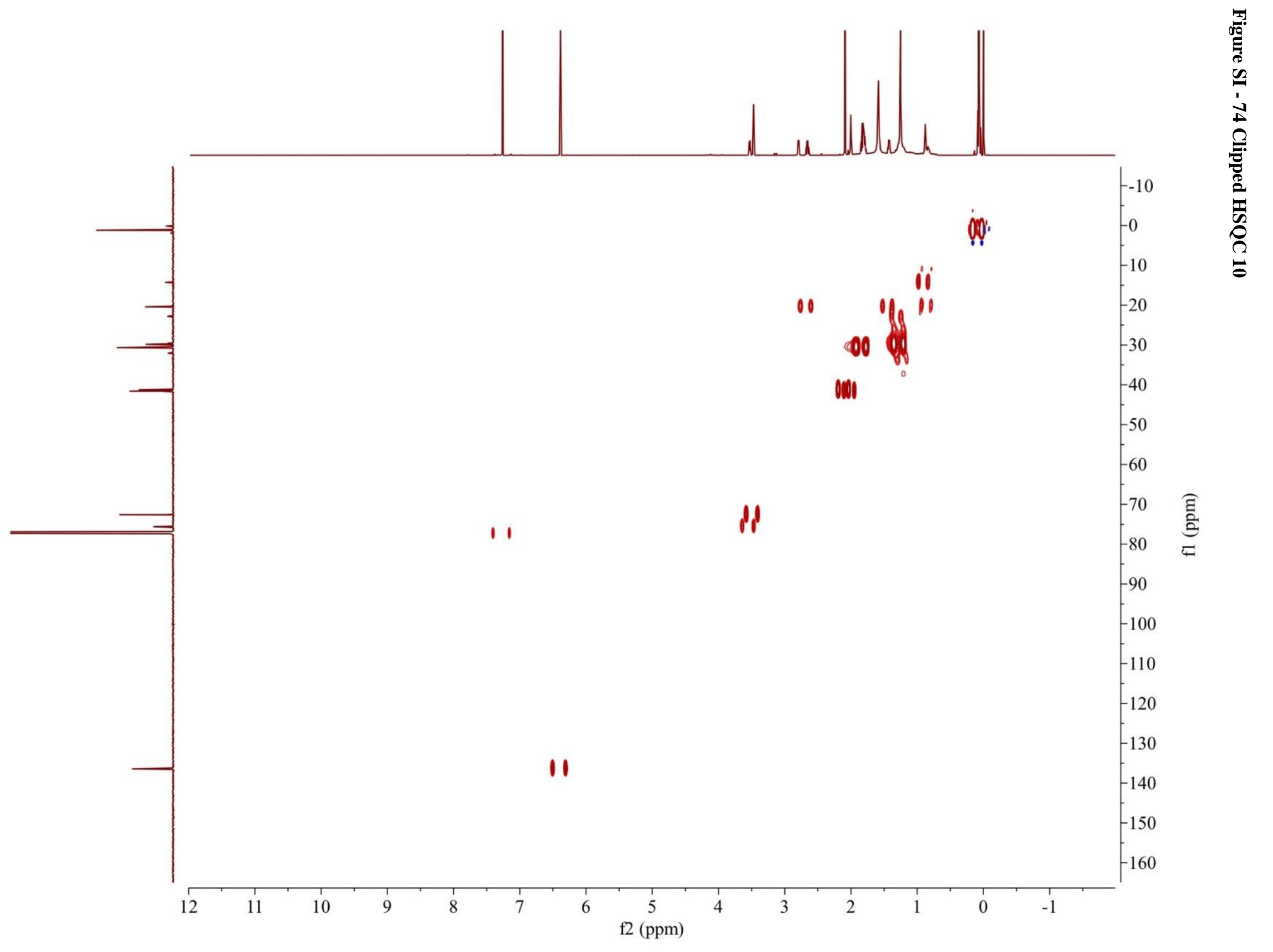




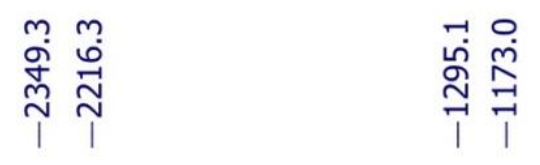

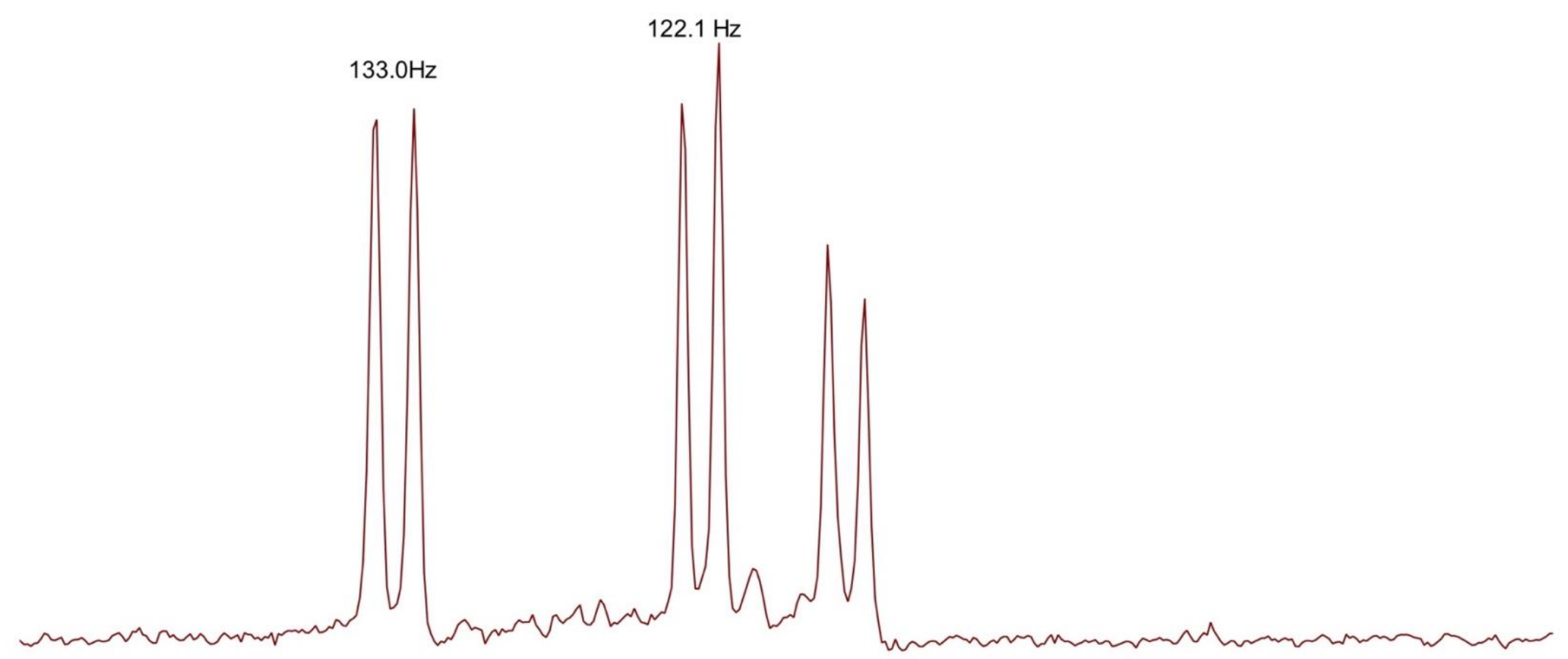




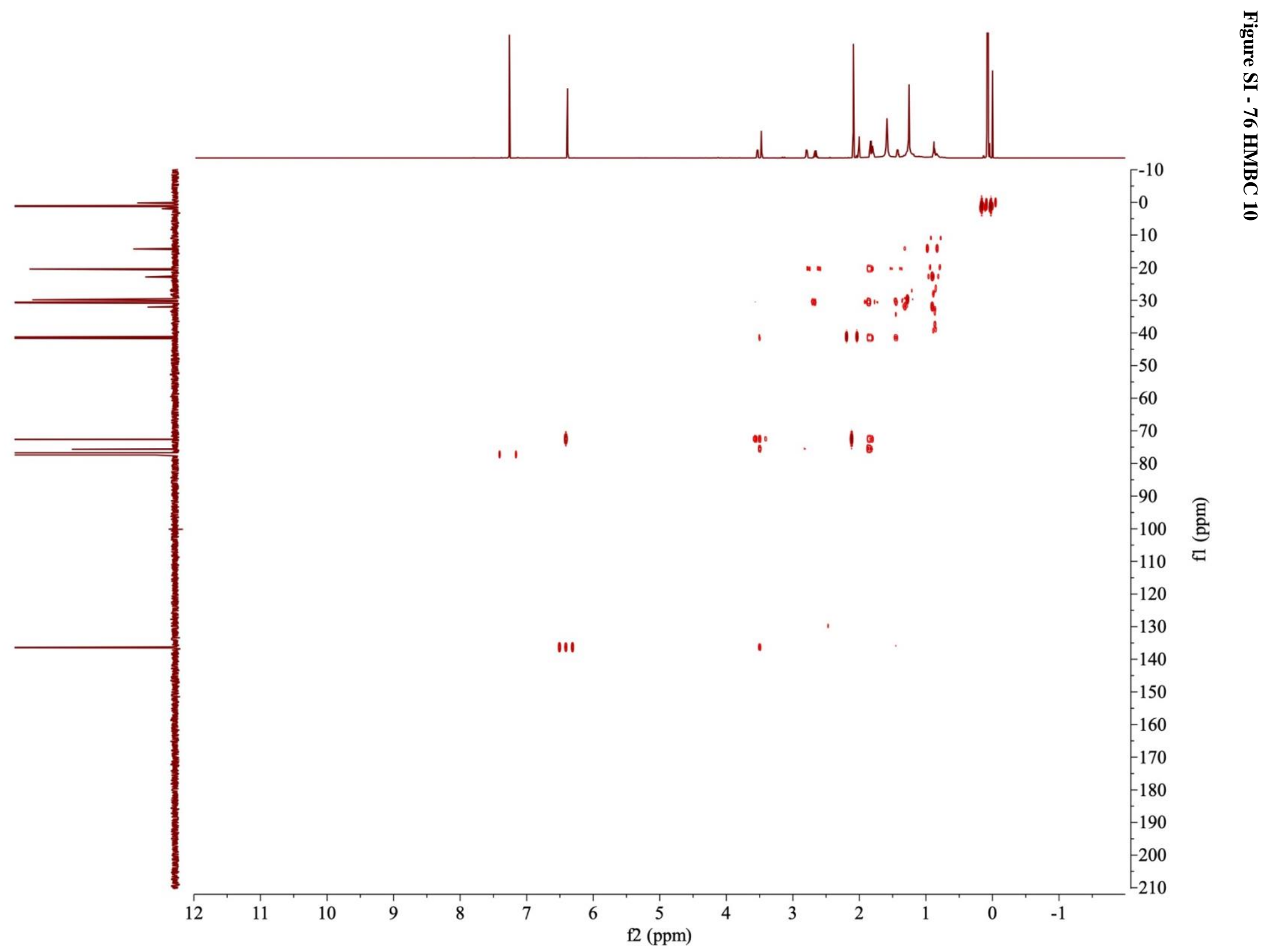




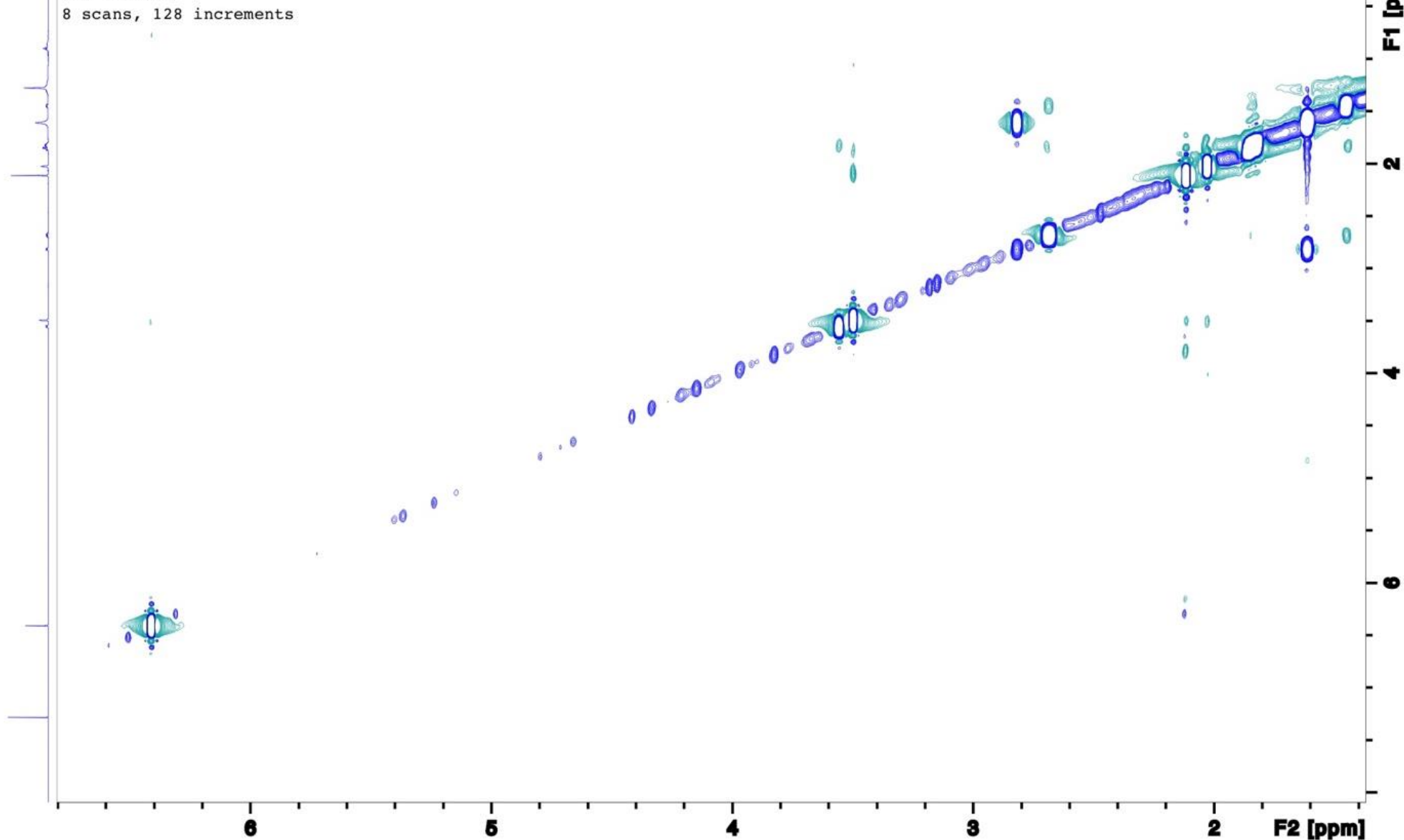




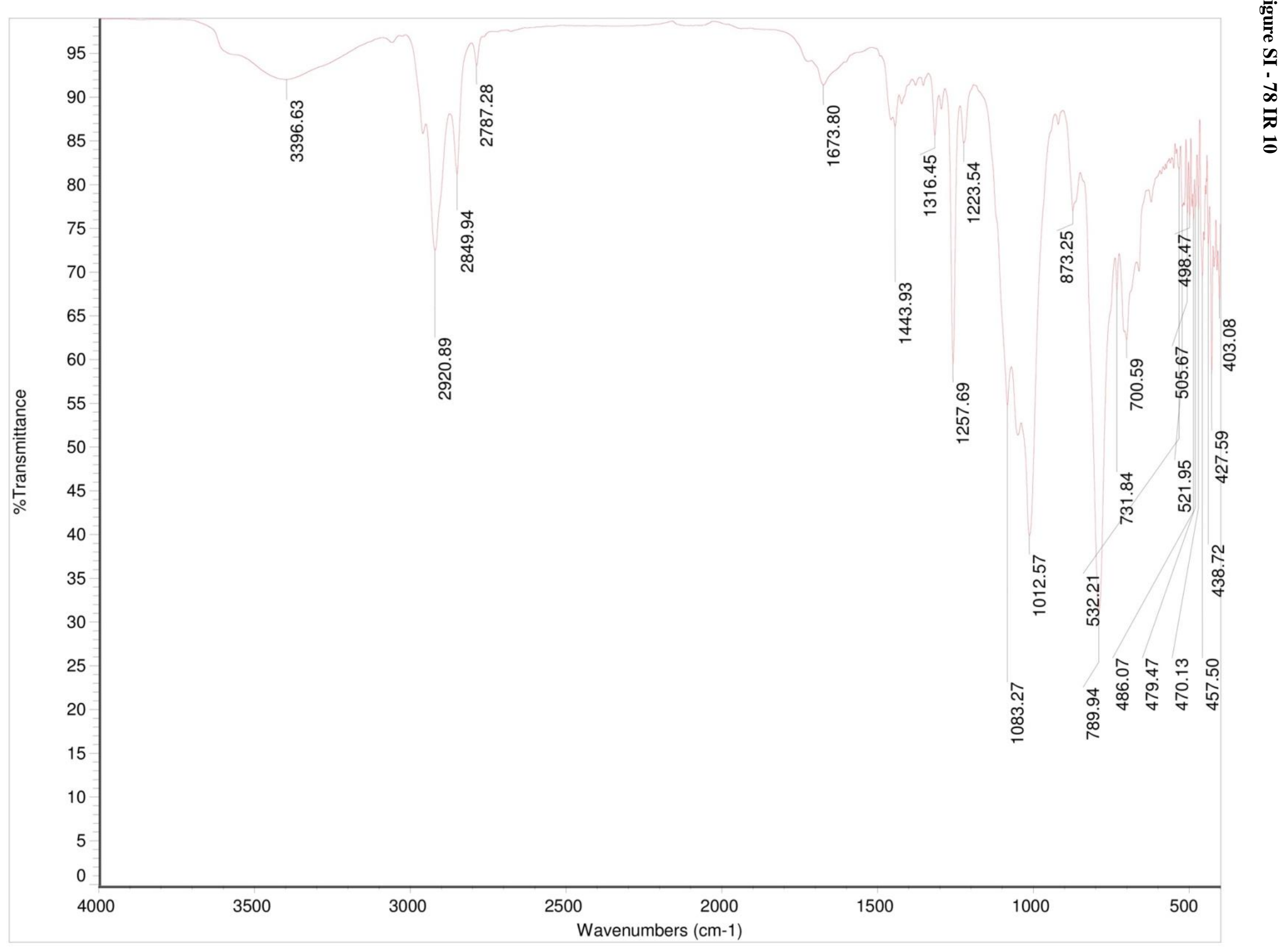




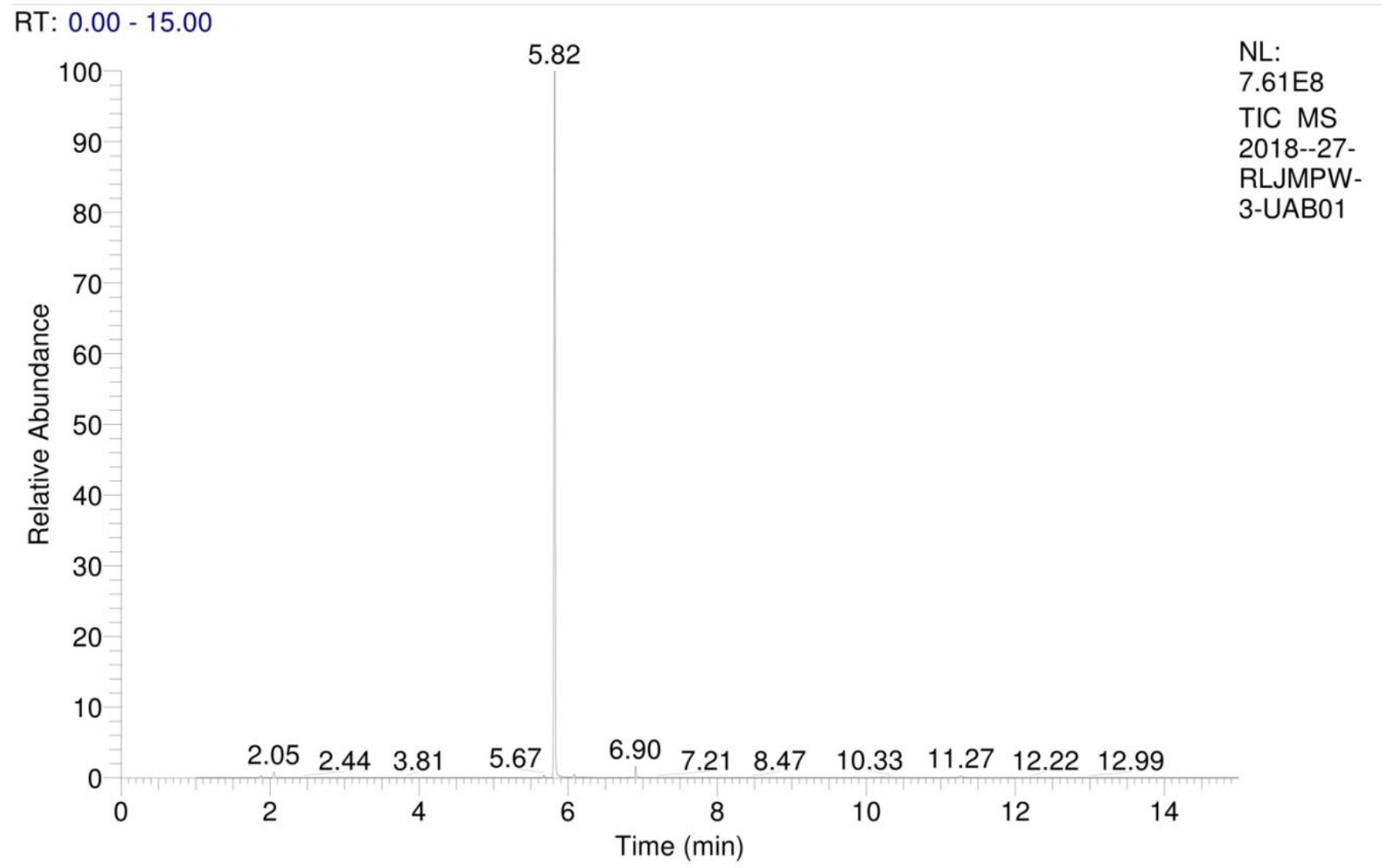

2018--27-RLJMPW-3-UAB01 \#1388-1429 RT: 5.72-5.86 AV: 42 NL: 1.41E7

T: $\{0,0\}+$ c El Full ms [120.00-500.00]

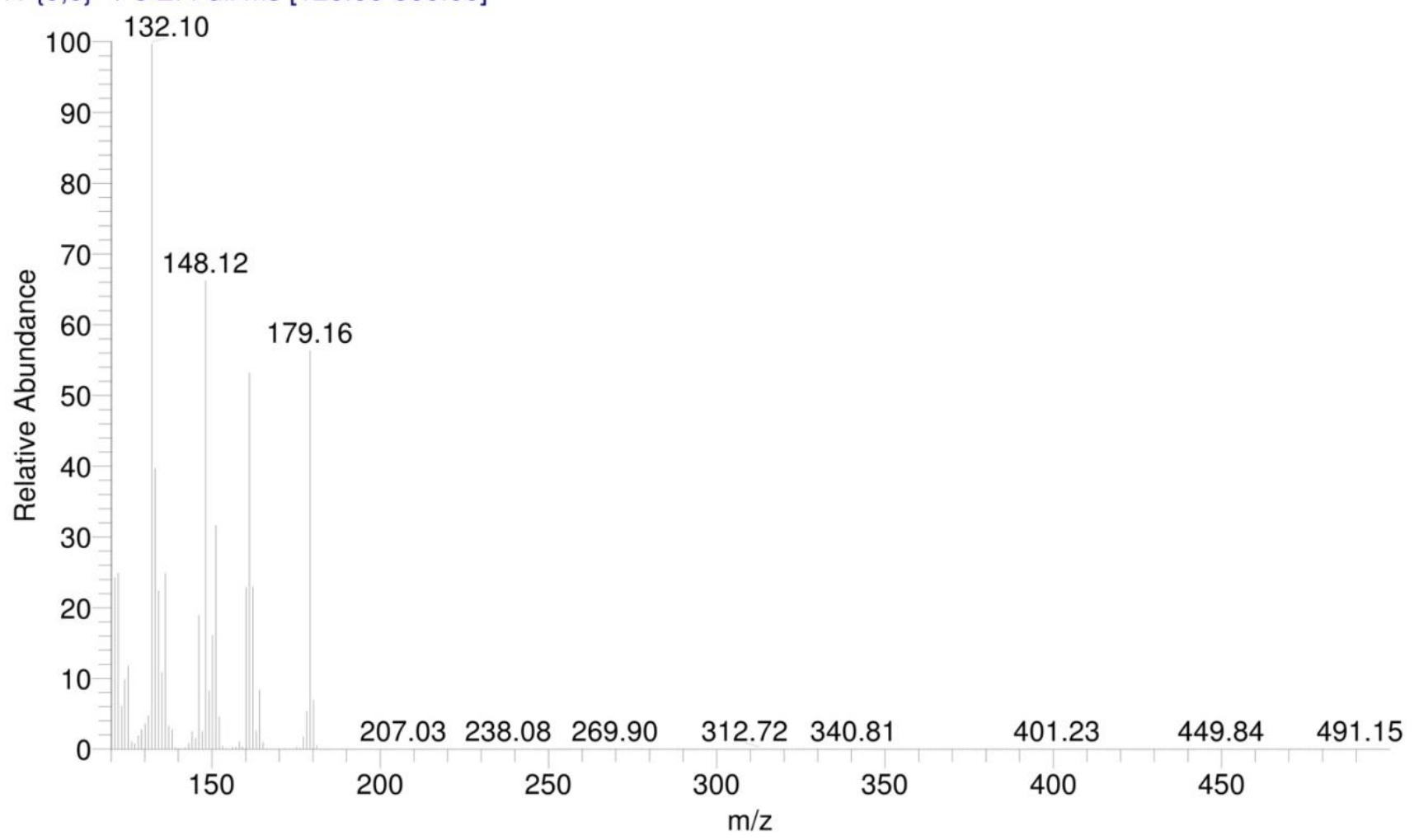


Figure SI - 80 High Resolution Mass Spectroscopy 10

Target Compound Screening Report

Results Acquired by The University of Texas at Austin Mass Spectrometry Facility

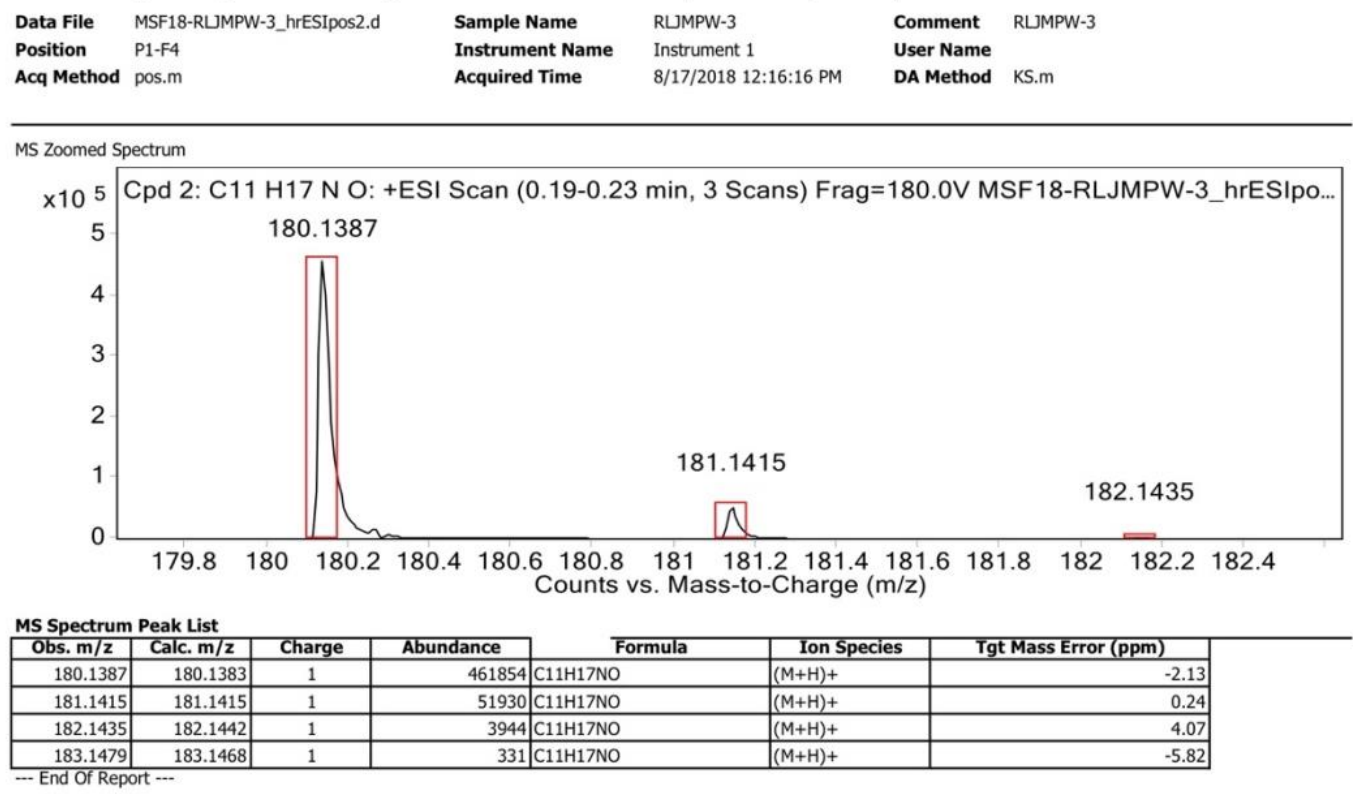




\section{Miscellaneous data}

Figure SI - 81. PERFECT-CLIP ZOOM of 1'@298 in CD2C12 (top), Tol-d8 (bottom)

\begin{tabular}{|ll|}
\hline \multicolumn{1}{|c|}{ Parameter } & \multicolumn{1}{c|}{ Value } \\
1 Title & $1^{\prime}$ \\
2 Solvent & CD2C12 \\
3 Temperature & 298 \\
4 Experiment & Perfect CLP \\
5 Spectrometer Frequency & $(850.21,213.80)$ \\
\hline
\end{tabular}

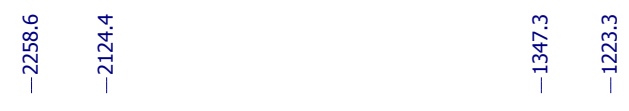

134.2 $\mathrm{PE}=10.2$

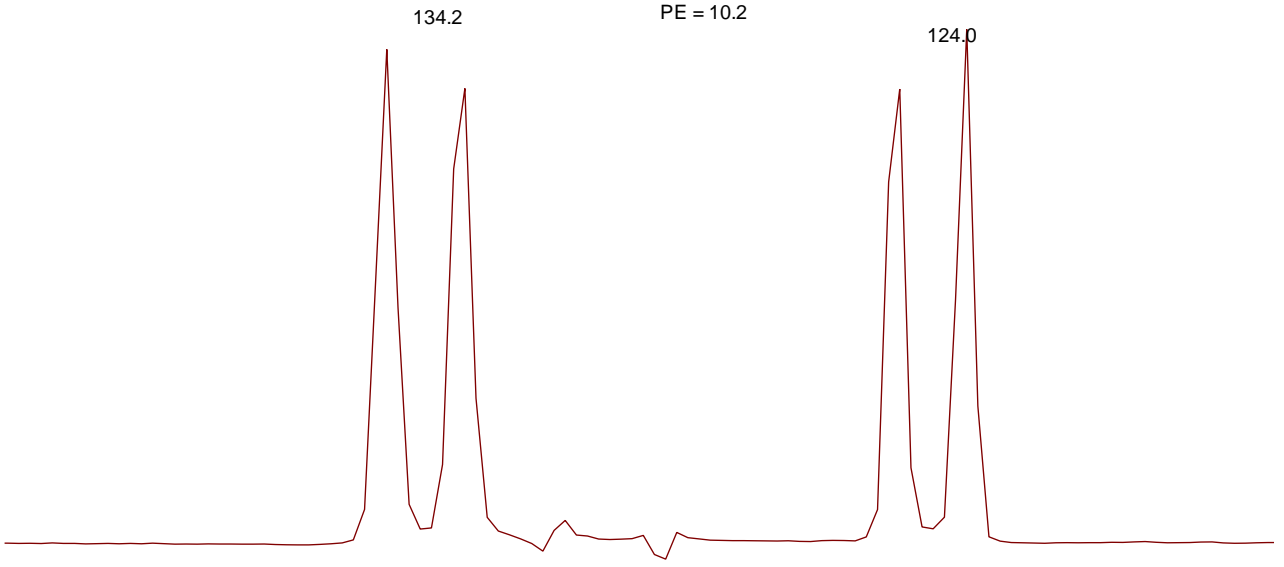

\section{f1 ( $\mathrm{ppm})$}

\begin{tabular}{|lll|}
\hline \multicolumn{1}{|c|}{ Parameter } & \multicolumn{1}{c|}{ Value } \\
1 & Title & $1^{\prime}$ \\
2 & Solvent & Tol \\
3 & Temperature & 298 \\
4 & Experiment & PERFECT CLIP \\
5 & Spectrometer Frequency & $(850.21,213.80)$ \\
\hline
\end{tabular}

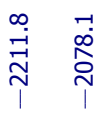

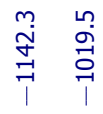

133.7

$P E=10.9$

122.8
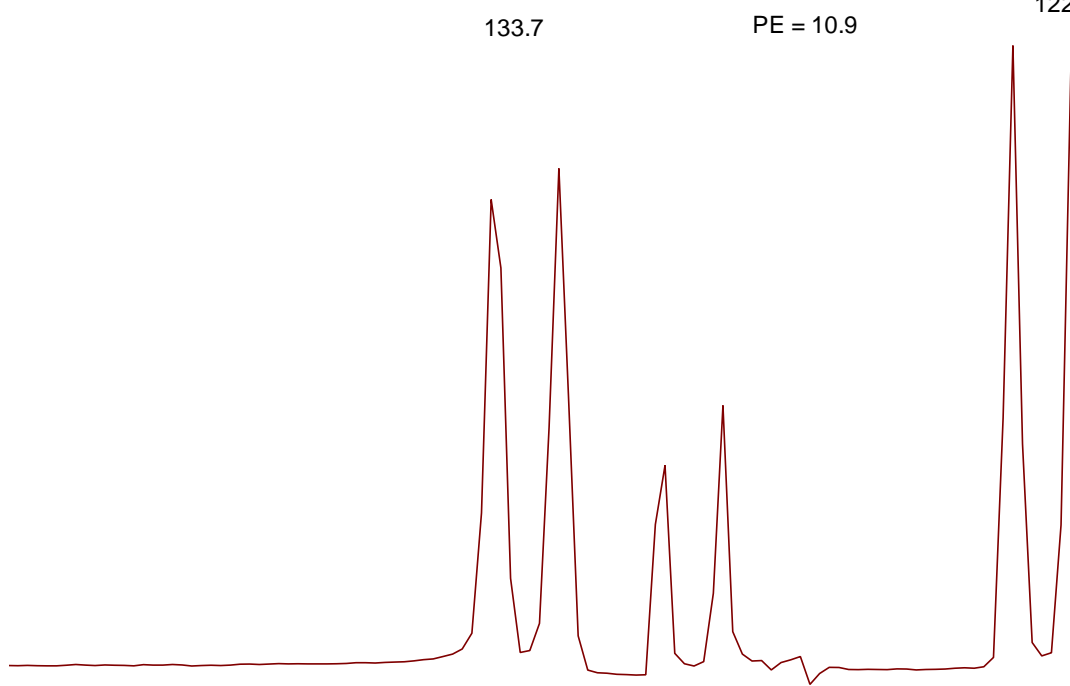
Figure SI - 82. PERFECT-CLIP ZOOM of 1' in CD2CL2@ 283K
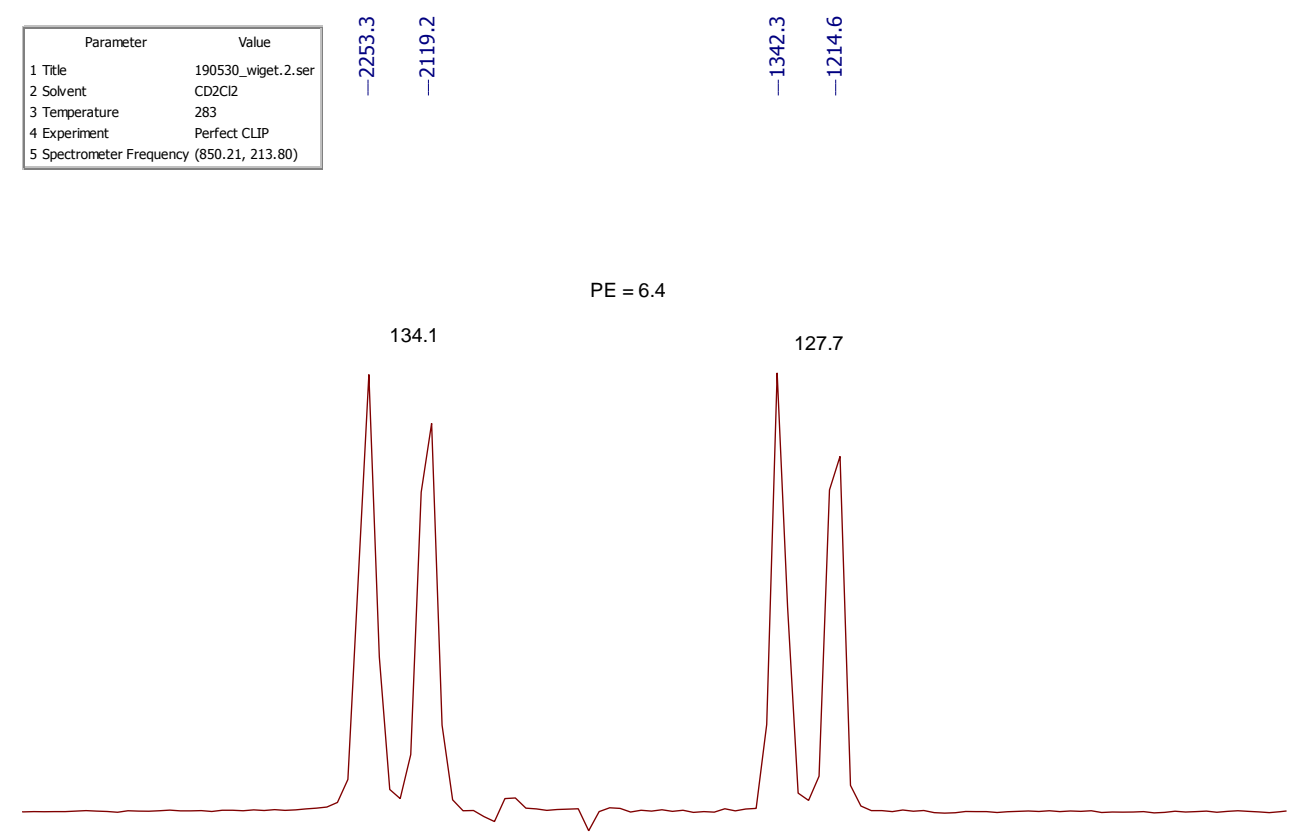

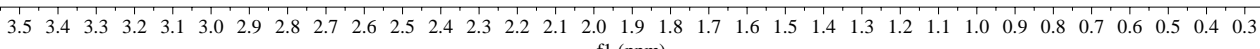
f1 (ppm) 
Figure SI - 83. PERFECT-CLIP of cyclohexanone (zoom) in CD2Cl2 @ 298K (top), 283K (bottom)

\begin{tabular}{|ll|}
\hline \multicolumn{1}{|c|}{ Parameter } & \multicolumn{1}{c|}{ Value } \\
1 Title & CHONE \\
2 Solvent & CD2C12 \\
3 Temperature & 298 \\
4 Experiment & PERFECT CLP \\
5 Spectrometer Frequency $(850.21,213.80)$ \\
\hline
\end{tabular}

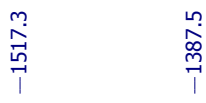

$129.8 \mathrm{~Hz}$

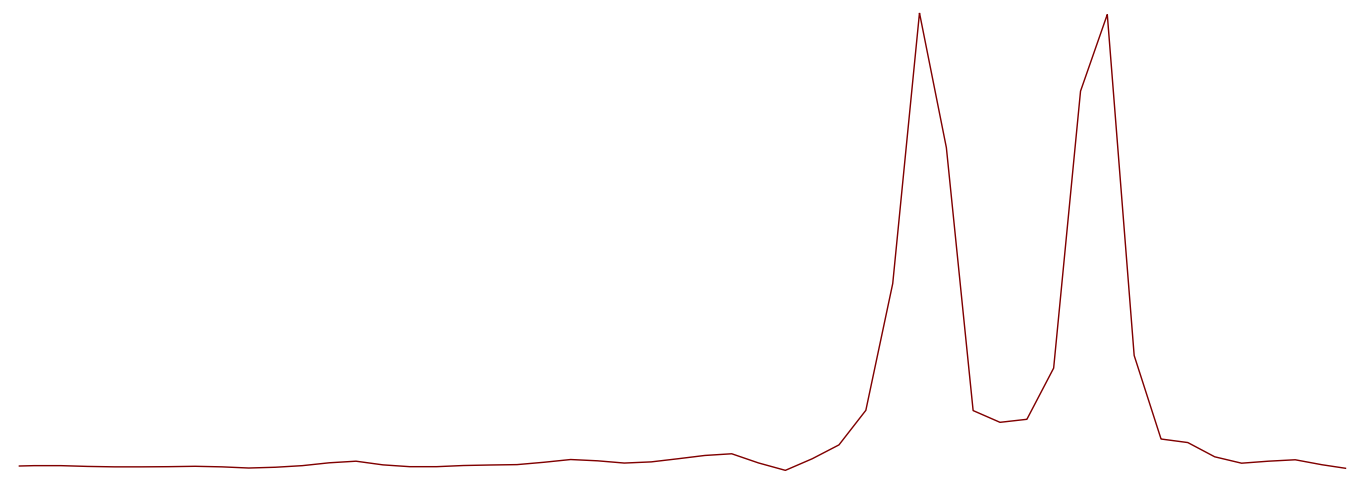

\begin{tabular}{llllllllllllllllllllllllllllll}
\hline 2.55 & 2.50 & 2.45 & 2.40 & 2.35 & 2.30 & 2.25 & 2.20 & 2.15 & 2.10 & 2.05 & 2.00 & 1.95 & 1.90 & 1.85 & 1.80 & 1.75 & 1.70 & 1.65 & 1.60 & 1.55 & 1.50 & 1.45
\end{tabular}

f1 (ppm)

\begin{tabular}{|ll|}
\hline \multicolumn{1}{|c|}{ Parameter } & \multicolumn{1}{c|}{ Value } \\
1 Title & CHONE \\
2 Solvent & CD2C12 \\
3 Temperature & 283 \\
4 Experiment & Perfect CLIP \\
5 Spectrometer Frequency & $(850.21,213.80)$ \\
\hline
\end{tabular}

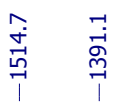

123.6

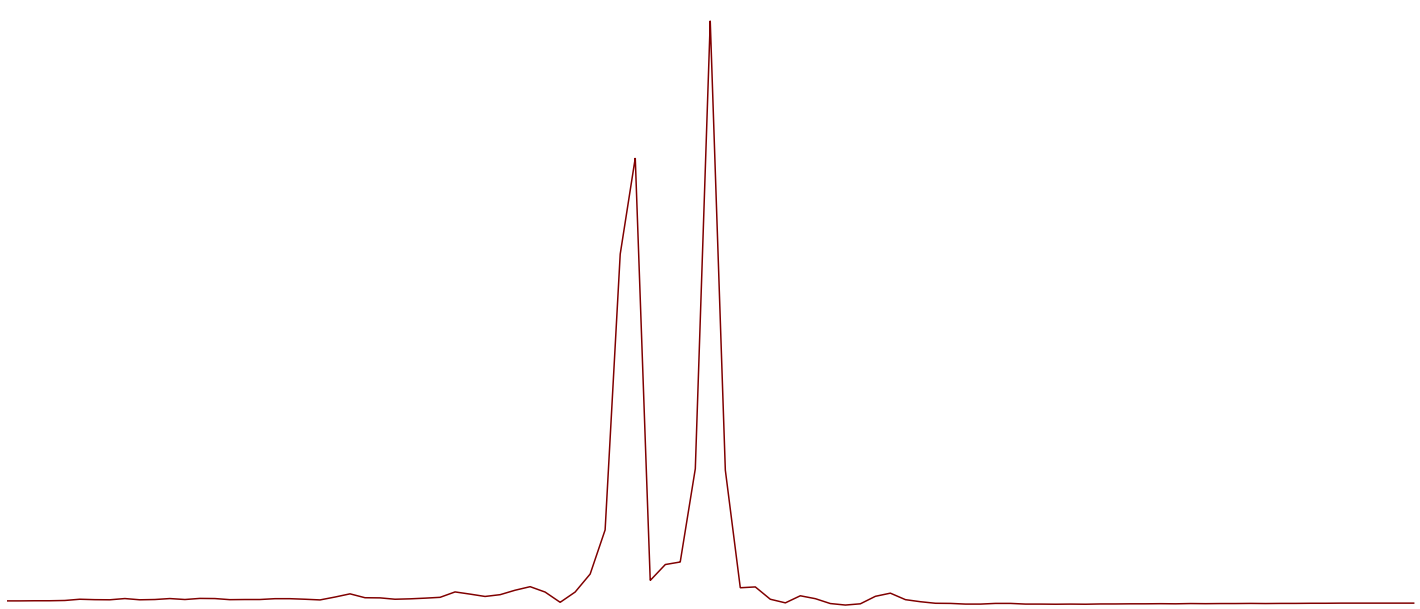

$\begin{array}{llllllllllllllllllllllllllllll}2.9 & 2.8 & 2.7 & 2.6 & 2.5 & 2.4 & 2.3 & 2.2 & 2.1 & 2.0 & 1.9 & 1.8 & 1.7 & 1.6 & 1.5 & 1.4 & 1.3 & 1.2 & 1.1 & 1.0 & 0.9 & 0.8 & 0.7 & 0.6 & 0.5 & 0.4\end{array}$ 
Figure SI - 84. PERFECT-CLIP of cyclohexanone (zoom) in Tol-d8 @ 298K (top), 283K (bottom)

\begin{tabular}{|ll|}
\hline \multicolumn{1}{|c|}{ Parameter } & \multicolumn{1}{c|}{ Value } \\
1 Title & CHONE \\
2 Solvent & Tol \\
3 Temperature & 298 \\
4 Experiment & PERFECT CLP \\
5 Spectrometer Frequency & $(850.21,213.80)$ \\
\hline
\end{tabular}

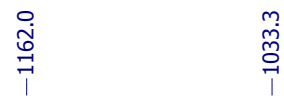

127.8

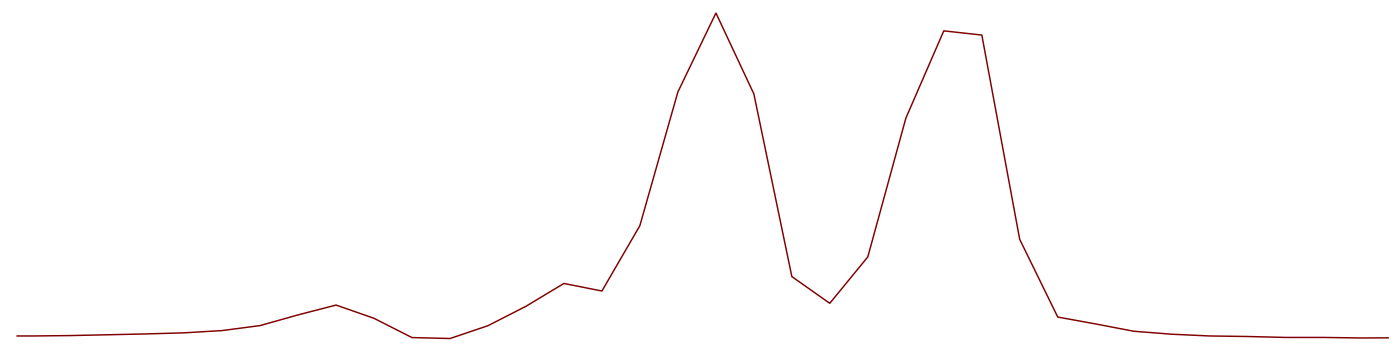

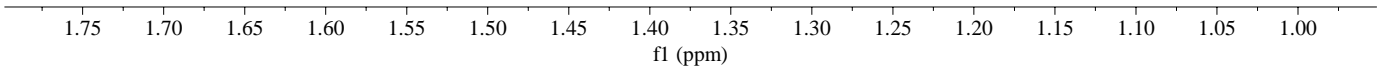

\begin{tabular}{|ll|}
\hline \multicolumn{1}{|c|}{ Parameter } & \multicolumn{1}{c|}{ Value } \\
1 Title & CHONE \\
2 Solvent & Tol \\
3 Temperature & 283 \\
4 Experiment & Perfect CLP \\
5 Spectrometer Frequency & $(850.21,213.80)$ \\
\hline
\end{tabular}

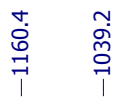

121.2

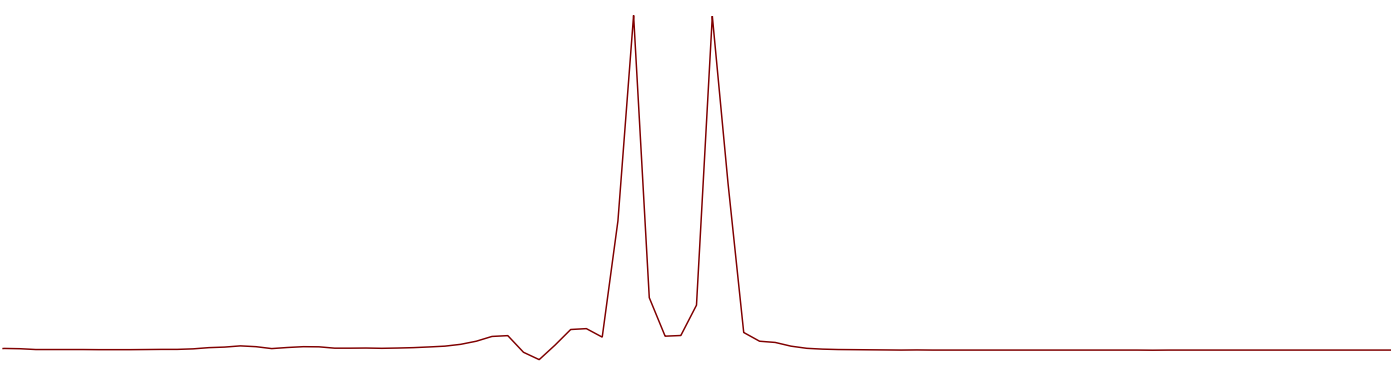

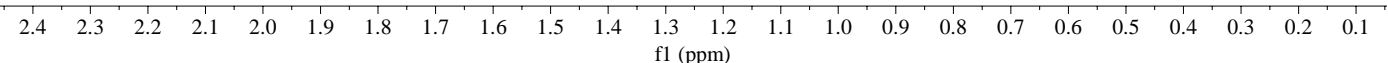


Figure SI - 85. PERFECT-CLIP cyclohexanol zoom in Tol-d8 @298K
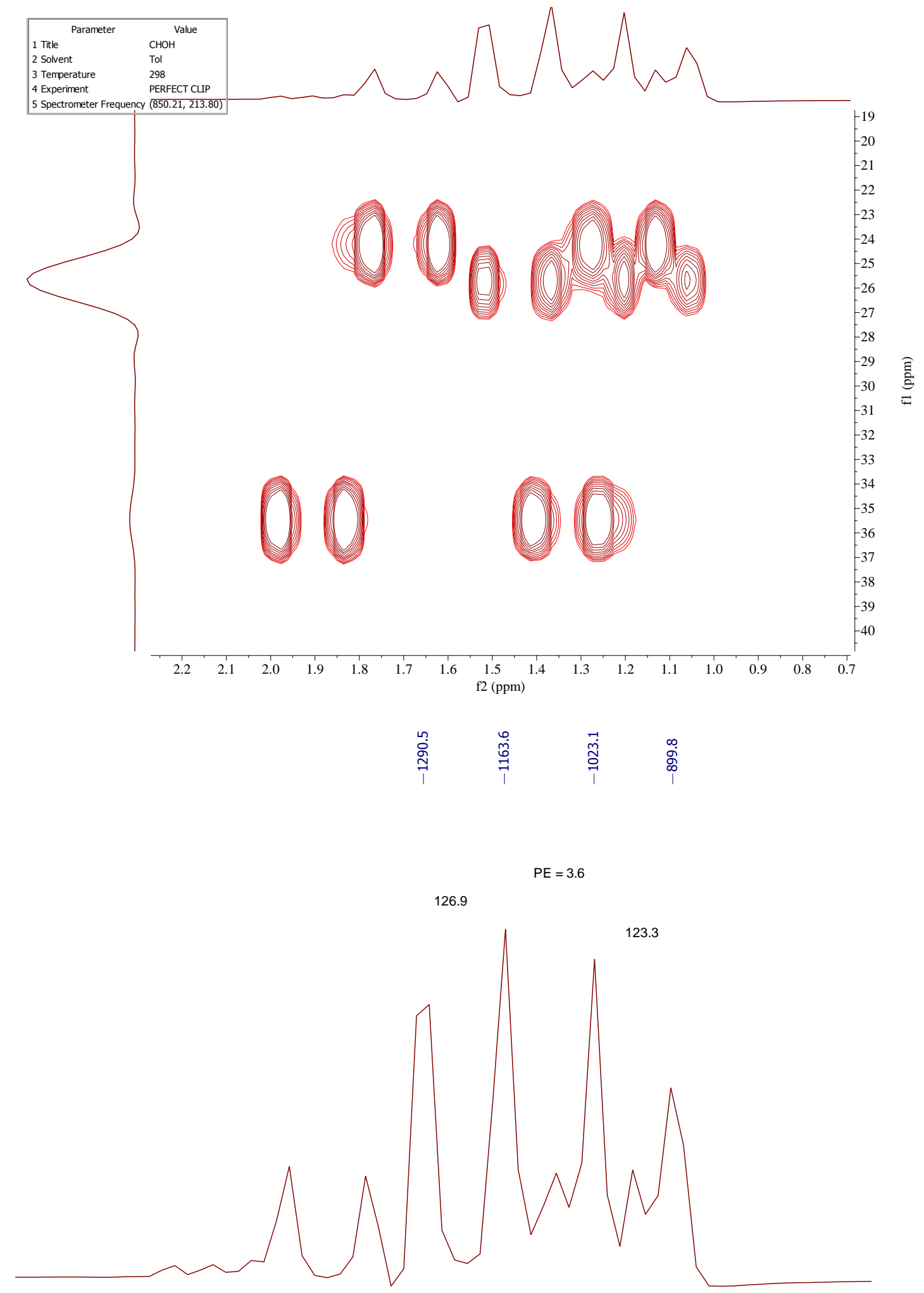

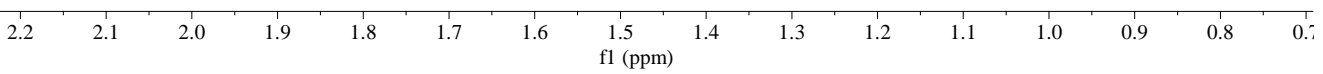


Figure SI - 86. PERFECT-CLIP of cyclohexanol in Tol-d8 @ 277K

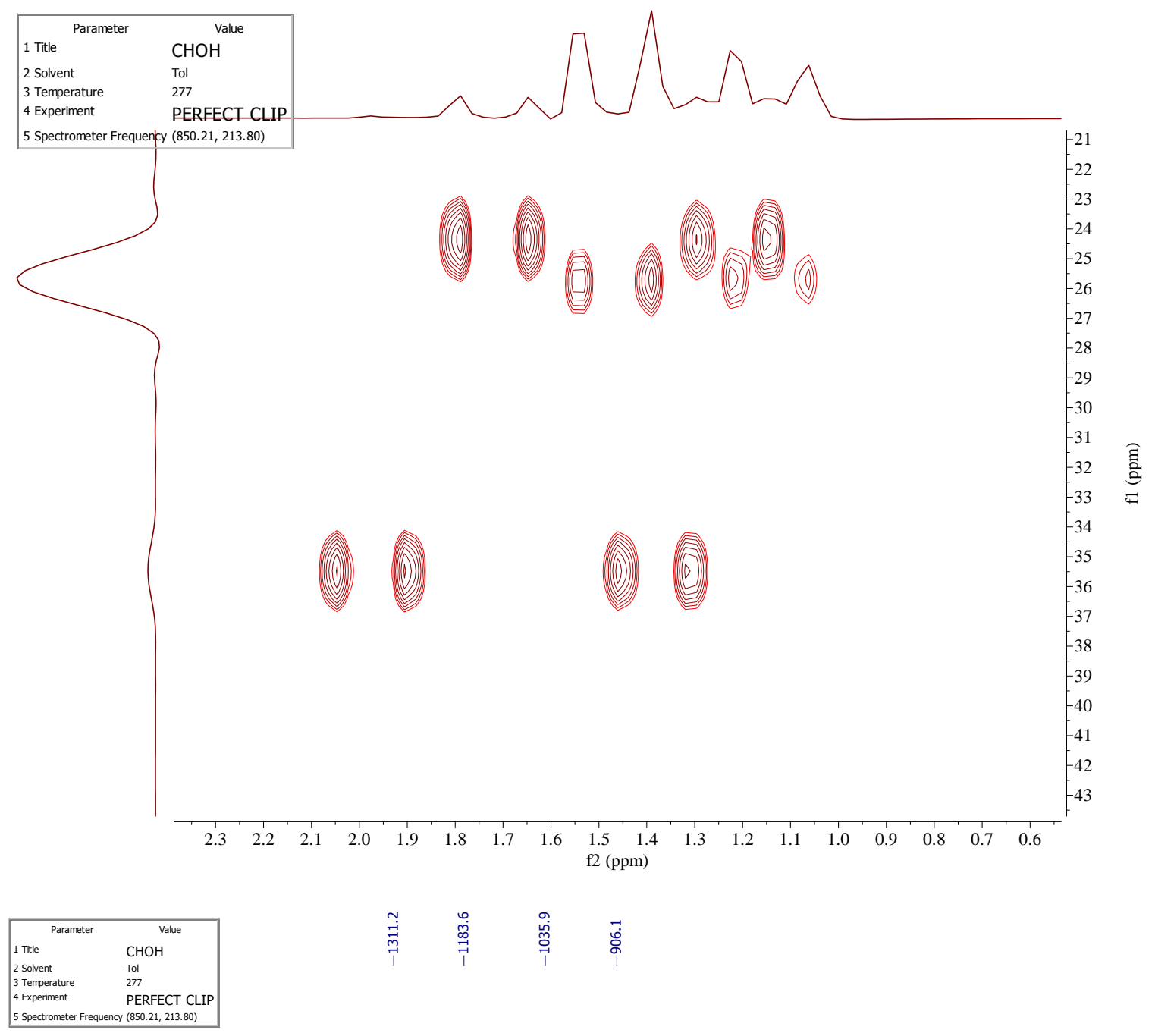

$\mathrm{PE}=2.2$

127.6

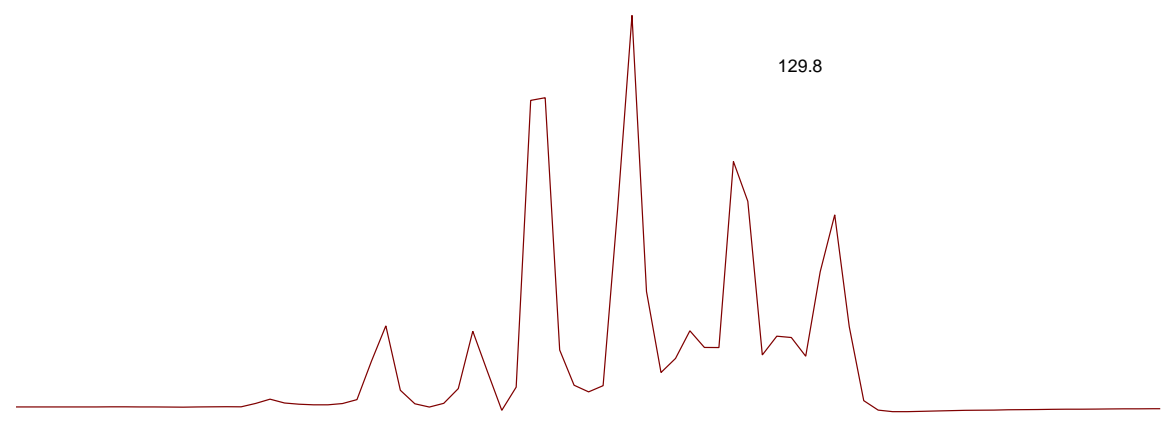

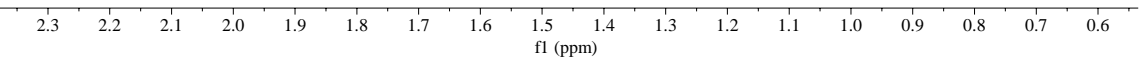


Figure SI - 87. PERFECT-CLIP of cyclohexanol in CD2C12 @ 283K

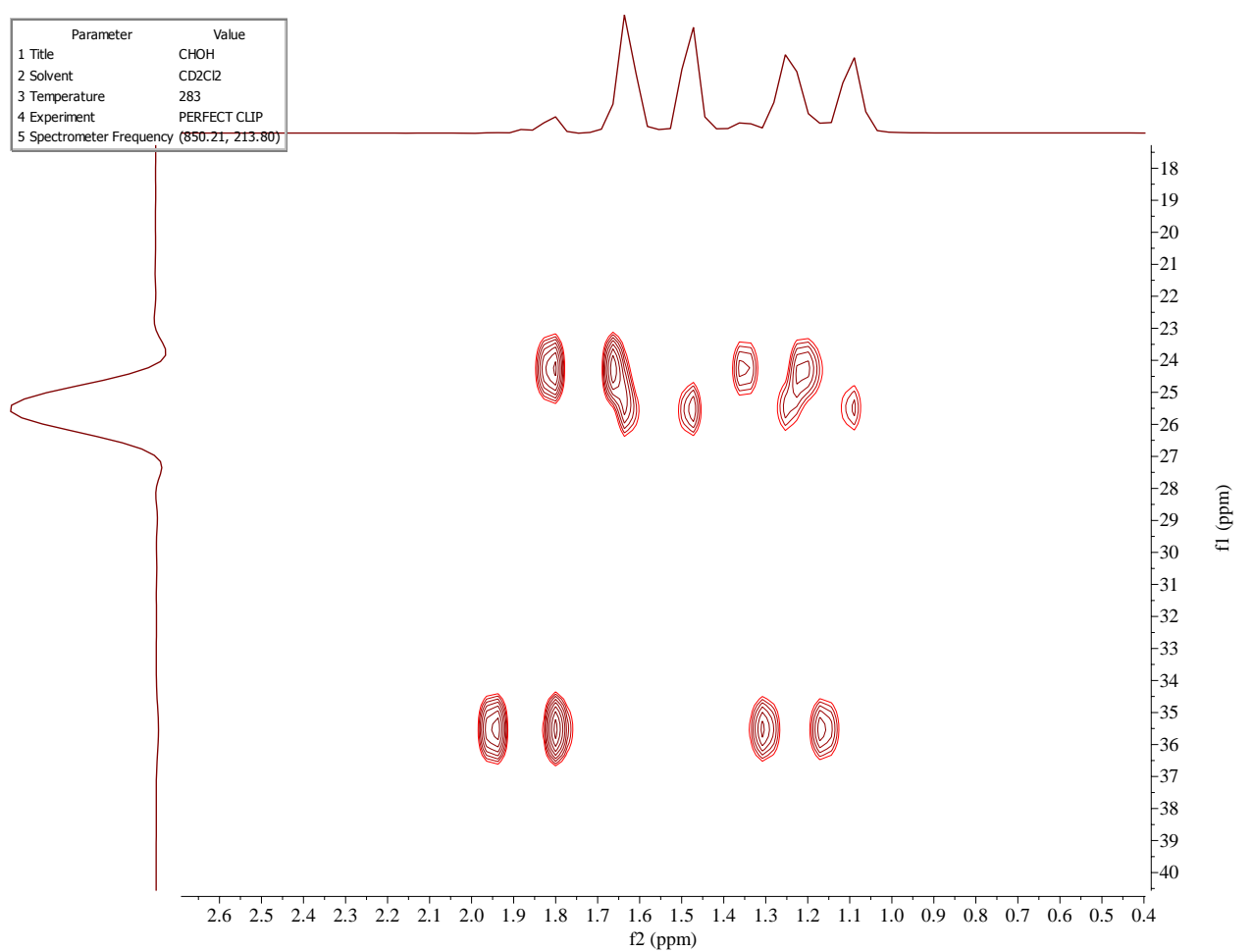

Figure SI - 88. PERFECT-CLIP zoom of cyclohexnol in Tol-d8 @ 283K

\begin{tabular}{|ll|}
\hline \multicolumn{1}{|c|}{ Parameter } & \multicolumn{1}{c|}{ Value } \\
1 Title & $\mathrm{CHOH}$ \\
2 Solvent & Tol \\
3 Temperature & 283 \\
4 Experiment & Perfect CLP \\
5 Spectrometer Frequency & $(850.21,213.80)$ \\
\hline
\end{tabular}

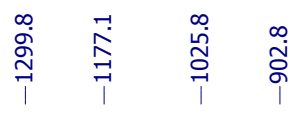

$\mathrm{PE}=0.3$

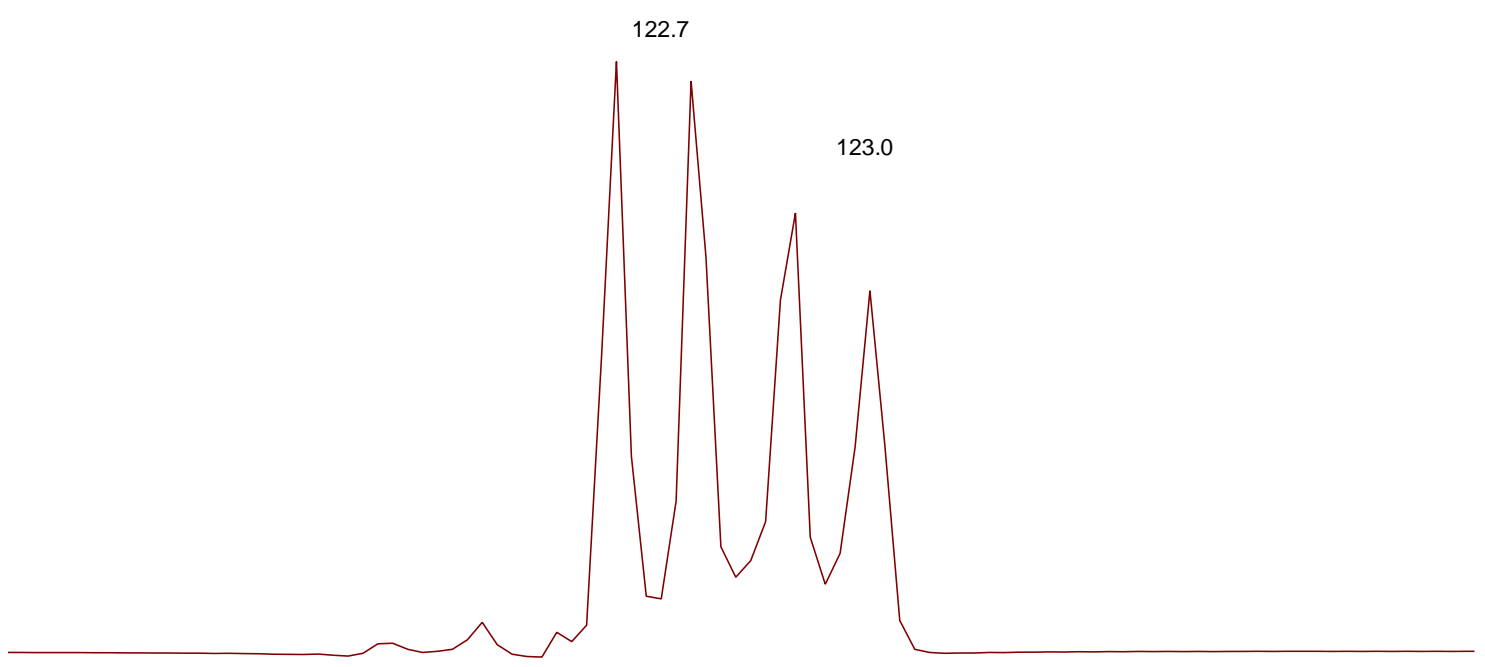

$\begin{array}{lllllllllllllllllllllllllllllll}2.6 & 2.5 & 2.4 & 2.3 & 2.2 & 2.1 & 2.0 & 1.9 & 1.8 & 1.7 & 1.6 & 1.5 & 1.4 & 1.3 & 1.2 & 1.1 & 1.0 & 0.9 & 0.8 & 0.7 & 0.6 & 0.5 & 0.4 & 0.3 & 0.2 & 0.1 & 0.0\end{array}$ 
Figure SI - 89. PERFECT-CLIP of cyclohexanol in CD2Cl2 @ 298K

\begin{tabular}{|ll|}
\hline \multicolumn{1}{|c|}{ Parameter } & \multicolumn{1}{c|}{ Value } \\
1 Title & $\mathrm{CHOH}$ \\
2 Solvent & $\mathrm{CD} 2 \mathrm{Cl} 2$ \\
3 Temperature & 298 \\
4 Experiment & Unknown \\
5 Spectrometer Frequency & $(850.21,213.80)$ \\
\hline
\end{tabular}

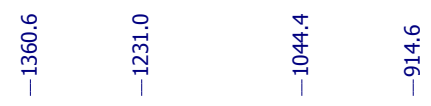

$\mathrm{PE}=0.3$

129.6

129.8

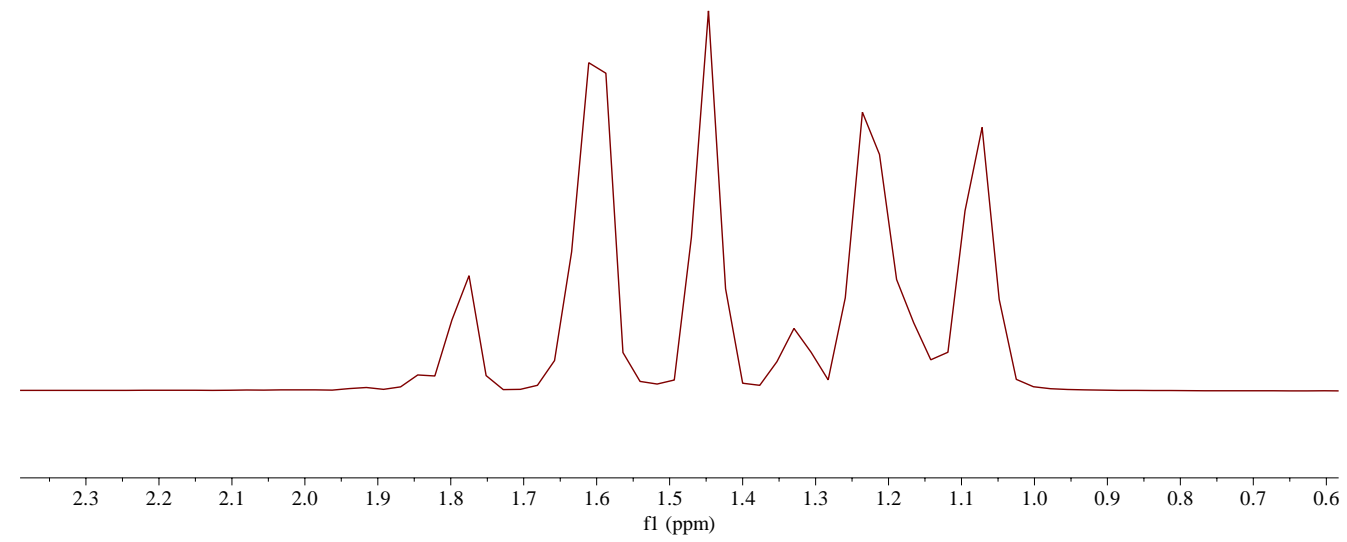

Figure SI - 90. Plot axial versus equatorial coupling constants in all compounds. Red compounds are from ref 4 a.

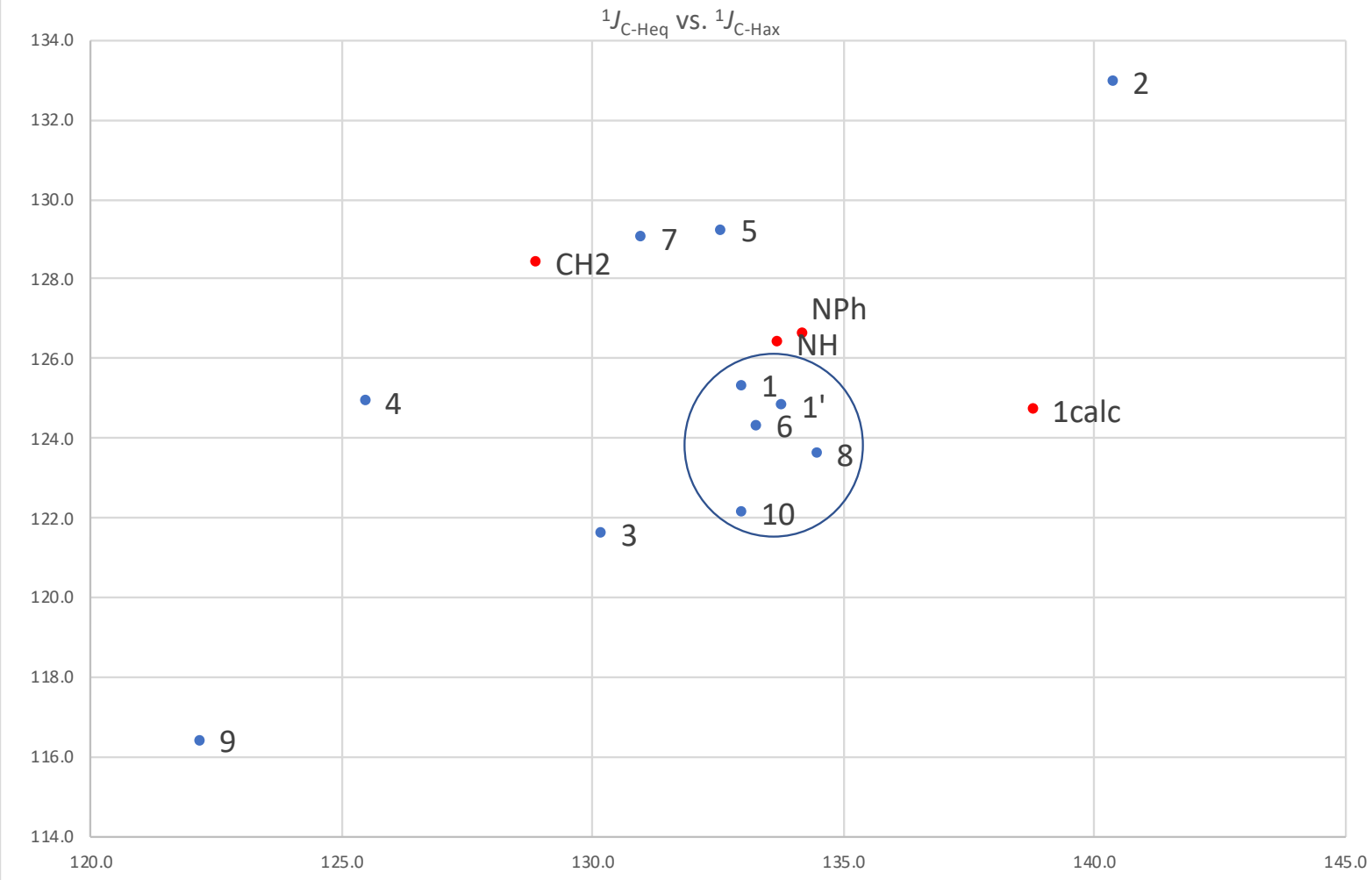

